TRANSACTIONS OF THE

AMERICAN MATHEMATICAL SOCIETY

Volume 351, Number 10, Pages 3869-3913

S 0002-9947(99)02138-8

Article electronically published on May 3, 1999

\title{
OVERGROUPS OF IRREDUCIBLE LINEAR GROUPS, II
}

\author{
BEN FORD
}

\begin{abstract}
Determining the subgroup structure of algebraic groups (over an algebraically closed field $K$ of arbitrary characteristic) often requires an understanding of those instances when a group $Y$ and a closed subgroup $G$ both act irreducibly on some module $V$, which is rational for $G$ and $Y$. In this paper and in Overgroups of irreducible linear groups, I (J. Algebra 181 (1996), 26-69), we give a classification of all such triples $(G, Y, V)$ when $G$ is a non-connected algebraic group with simple identity component $X, V$ is an irreducible $G$ module with restricted $X$-high weight(s), and $Y$ is a simple algebraic group of classical type over $K$ sitting strictly between $X$ and SL( $V)$.
\end{abstract}

\section{INTRODUCTION}

E. B. Dynkin in 1957 [3, 2] classified the maximal closed connected subgroups of simple algebraic groups when the underlying (algebraically closed) field has characteristic 0 . Seitz $([9,10])$ and Testerman $([13])$ completed the same program in positive characteristic in the 1980's. Their analyses for the classical group cases were based primarily on a striking result: If $G$ is a simple algebraic group and $\varphi: G \rightarrow \mathrm{SL}(V)$ is a tensor indecomposable irreducible rational representation, then with specified exceptions the image of $G$ is maximal among closed connected subgroups of one of the classical groups $\mathrm{SL}(V), \mathrm{Sp}(V)$, or $\mathrm{SO}(V)$. What is most striking is the brevity of the list of exceptions.

From a slightly different perspective, the question these authors answered was: Given a closed, connected subgroup $G$ of $\operatorname{SL}(V)$ for some vector space $V$, with $G$ acting irreducibly on $V$, find all possibilities for closed, connected overgroups $Y$ of $G$ in $\operatorname{SL}(V)$.

This question of irreducible overgroups appears in other contexts as well, sometimes for non-connected subgroups. Here and in [4] we present results for some such non-connected subgroups, namely, those with simple identity components. The overall program is to classify all possible triples $(G, Y, V)$ with $G$ and $Y$ both closed subgroups of $\mathrm{SL}(V)$ acting irreducibly on $V, G<\operatorname{Aut}(Y), Y \neq \mathrm{SL}(V), \mathrm{SO}(V)$, or $\operatorname{Sp}(V)$, and $Y$ a simple group of classical type (the corresponding question for $Y$ of exceptional type is also open). We give complete results for the case when $G$ is not connected but has simple identity component $X$ and the $T_{Y}$-high weight and $T_{X}$-high weights of $V$ are restricted. Specifically, the papers are concerned with the proof of Theorem 1.

Received by the editors August 18, 1995 and, in revised form, April 30, 1997.

1991 Mathematics Subject Classification. Primary 20G05.

Supported in part by the NSF and the NSA. 
Theorem 1. Let $G$ be a non-connected algebraic group, over a field $K$ of arbitrary characteristic $p \geq 0$, with simple identity component $X$. Let $V$ be an irreducible $K G$-module with restricted $X$-high weight(s). Let $Y$ be a simple algebraic group of classical type such that $X<Y<\mathrm{SL}(V)$ and $G \leq \operatorname{Aut}(Y)$. Then $\left.V\right|_{Y}$ is irreducible with restricted high weight if and only if $Y=\mathrm{SO}(V), Y=\mathrm{Sp}(V)$, or $(X, Y, V)$ appears in Table 1 or Table 2, see $\S 6$.

If $G$ has simple identity component $X$, then $G \leq \operatorname{Aut}(X)$ (there is also the possibility of a torus acting as scalars on each of the $X$-composition factors of $V$; we ignore this trivial possibility). Since we require that $G \neq X$, we therefore may restrict our attention to $X$ of type $A_{m}, D_{m}$, or $E_{6}$. We assume henceforth that $Y$ is simply connected and that $X$ and $Y$ act on $W$, the natural module for $Y$.

The analysis is different depending on whether $X$ acts reducibly or irreducibly on $W$. We settled the reducible case in [4], and we consider the irreducible case here. Also, we will assume here that the involutory graph automorphism of $X$, if it is in $G$, also acts on $W$ (though it need not be in $Y$ ), as we dealt with the case when it does not act on $W$ in the final section of [4].

If $\left.V\right|_{X}$ is irreducible, then we are in the case examined by Seitz in [9], with the additional condition that $X$ have an outer automorphism which acts on $V$. We examine Table 1 of that paper, and find that we have such a situation in the examples there labelled $\mathrm{I}_{4}, \mathrm{I}_{5}, \mathrm{I}_{6}$ for $n=3, \mathrm{II}_{1}, \mathrm{~S}_{1}, \mathrm{~S}_{8}$ (in $\mathrm{S}_{8}$ we could take $G=X\langle t\rangle$, $G=X\langle s\rangle$, or $G=X\langle s, t\rangle$, where $t, s$ are outer automorphisms of $X$ of order 2 and 3 respectively), and $\mathrm{MR}_{4}$. These examples are collected in Table 1, and henceforth we shall assume that $\left.V\right|_{X}$ is reducible.

1.1. Notation and Conventions. All structures are assumed to be constructed over the same algebraically closed field $K$, of characteristic $p \geq 0$. Throughout, $X$ will denote a simple algebraic group over $K$ admitting an outer automorphism (so $X$ is of type $A_{m}, D_{m}$, or $E_{6}$ ). A fixed standard graph automorphism of order 2 will be denoted by $t$, and if $X$ has an outer automorphism of order 3 (i.e. if $X=D_{4}$ ), we will fix one and denote it by $s$. Thus $G$ is $X\langle t\rangle$ except possibly when $X=D_{4}$, in which case we also consider $G=X\langle s\rangle$ and $G=X\langle s, t\rangle$.

For any reductive group $H$ we consider, with fixed maximal torus $T, \Sigma(H)$ will denote the roots of $H$ relative to $T$. If $\gamma \in \Sigma(H)$, we let $h_{\gamma}: K^{*} \rightarrow T$ be the one-parameter subgroup of $T$ such that $\alpha\left(h_{\gamma}(x)\right)=x^{<\alpha, \gamma>}$ for any $\alpha \in \Sigma(H)$ and $x \in K^{*}$.

We let $B_{X}$ be a fixed $t$-stable Borel subgroup of $X$, containing a fixed $t$-stable maximal torus $T_{X}$. Define sets of simple roots $\left\{\beta_{1}, \beta_{2}, \ldots, \beta_{m}\right\}=\Pi(X) \subseteq \Sigma(X)$ and fundamental dominant weights $\left\{\delta_{1}, \ldots, \delta_{m}\right\}$ with respect to $T_{X}$ and $B_{X}$ but with the opposite of the standard convention: The set of positive roots $\Sigma^{+}(X)$ is defined by $B_{X}=U_{X} T_{X}$ where $U_{X}=\prod U_{-\alpha}$ for $\alpha \in \Sigma^{+}(X)$. Then for $J \subseteq \Pi(X)$, $P_{X}$ is the opposite of the standard parabolic corresponding to $J$. We assume the $\delta_{i}$ are numbered so that $\delta_{i}$ corresponds to $\beta_{i}$ for every $i$.

The group $Y$ will be a simple algebraic group over $K$ of classical type and rank $n\left(A_{n}, B_{n}, C_{n}\right.$ or $\left.D_{n}\right)$, such that $X<Y$ and $G \leq \operatorname{Aut}(Y)$. Let $\left\{\alpha_{1}, \alpha_{2}, \ldots, \alpha_{n}\right\}=$ $\Pi(Y)$ be a set of simple roots of $Y$ and $\left\{\lambda_{i}\right\}$ be the set of fundamental dominant weights such that $\lambda_{i}$ corresponds to $\alpha_{i}$. Notation and conventions similar to those used for $X$ are used for parabolic subgroups of $Y$.

For a group $H$ acting on a module $M,\left[M, H^{l}\right]$ will denote the $l$-fold commutator of $H$ with $M$. 
The $K$-vector space $V$ is assumed to be a restricted irreducible $Y$-module with high weight $\lambda=\sum a_{i} \lambda_{i}$, such that $V$ is irreducible as a $G$-module but not as an $X$ module (see the comment at the end of the previous subsection). We assume that the $T_{X}$-high weights of $V$ are restricted as well. So if $G=X\langle t\rangle$, then $\left.V\right|_{X}=V_{1} \oplus V_{2}$, where each of $V_{1}, V_{2}$ is a restricted irreducible $X$-module.

The natural module for $Y$ will be denoted by $W$. We assume that $W$ is irreducible as an $X$-module, and $\delta$ will denote its $T_{X}$-high weight. As in [4], we will always assume that $Y$ is the smallest of $\mathrm{SL}(W), \mathrm{SO}(W), \mathrm{Sp}(W)$ containing $X$.

Finally, we assume that $G$ acts on $W$, as the case when it does not was considered in $[4]$.

We label Dynkin diagrams for the groups we will be dealing with as follows, and we always number fundamental roots and fundamental dominant weights to agree with this labelling:

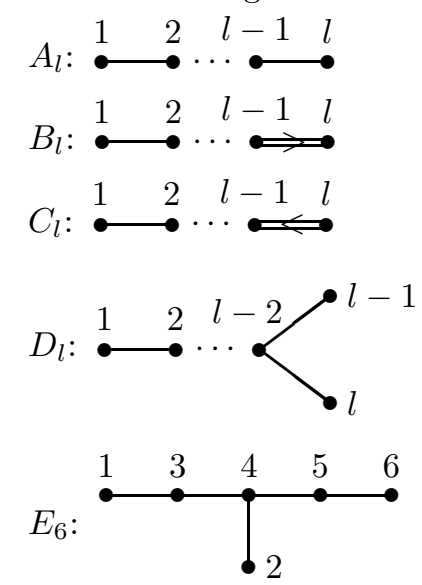

We will sometimes use the standard partial order on weights: $\nu \succ \mu$ if and only if $\nu-\mu$ is a sum of positive roots.

\section{2. $Q_{X}$-LEVELS AND EMBEDDINGS OF PARABOLICS}

In this section we introduce important facts about the "commutator series" of a module of a simple algebraic group.

Lemma 2.1. If $H$ is a simple algebraic group whose root system has only one root length, then restricted irreducible $H$-modules are tensor indecomposable (in particular, restricted irreducible $X$-modules are tensor indecomposable).

Proof. This is part of 1.6 of [9].

Lemma 2.2. Let $M$ be an irreducible restricted $H$-module with high weight $\gamma$ for some simple algebraic group $H$. Let $P$ be a proper parabolic subgroup of $H$, with $P=Q L$ a Levi decomposition. Then $M /[M, Q]$ is irreducible for $L$ and for $L^{\prime}=$ $[L, L]$, with $T_{L^{\prime}}$-high weight $\left.\gamma\right|_{T_{L^{\prime}}}$.

Proof. This is 1.7 and 2.1 of [9].

Let $H, M, \gamma$, and $P$ be as in the last lemma. Let $\left\{\epsilon_{i}\right\}$ be the set of fundamental roots of $H$. 
Definition 2.3. Let $\mu$ be a weight of $M$, say $\mu=\gamma-\sum c_{i} \epsilon_{i}$, with each $c_{i} \geq 0$. The $Q$-level of $\mu$ is $\sum c_{j}$, where the sum ranges over those $j$ for which $\epsilon_{j} \in \Pi(H)-\Pi\left(L^{\prime}\right)$. The $Q$-level $l$ of $M$ is the sum of weight spaces for weights having $Q$-level $l$ and is denoted $M_{l}$.

Lemma 2.4. Let $H, M$, and $P$ be as above. If $H$ is simply laced or if $p>2(p>3$ for $\left.H=G_{2}\right)$, then

1. $\left[M, Q^{l}\right]=\bigoplus M_{\mu}$, the sum taken over those weights $\mu$ having $Q$-level at least $l$.

2. $\left[M, Q^{l}\right] /\left[M, Q^{l+1}\right] \cong M_{l}$.

3. $\operatorname{dim}\left(\left[M, Q^{l}\right] /\left[M, Q^{l+1}\right]\right) \leq s \cdot \operatorname{dim}\left(\left[M, Q^{l-1}\right] /\left[M, Q^{l}\right]\right)$, where $s$ is the number of positive roots $\beta$ such that $U_{-\beta} \leq Q$ and $\beta=\epsilon_{i}+\beta^{\prime}$ for some $\epsilon_{i} \in \Pi(H)-\Pi\left(L^{\prime}\right)$, with $\beta^{\prime}=0$ or a sum of roots in $\Pi\left(L^{\prime}\right)$.

4. $\operatorname{dim}\left(\left[M, Q^{l}\right] /\left[M, Q^{l+1}\right]\right) \leq \operatorname{dim}(Q) \cdot \operatorname{dim}\left(\left[M, Q^{l-1}\right] /\left[M, Q^{l}\right]\right)$.

Proof. This is 2.3 of [9].

We will write $M^{l}(Q)$ for the quotient $\left[M, Q^{l-1}\right] /\left[M, Q^{l}\right]$.

Lemma 2.5. Let $H=A_{l}$; let $c$ be an integer such that $0<c<p$; and let $\gamma_{1}, \ldots, \gamma_{l}$ be the fundamental dominant weights for $H$. The irreducible module $M$ having high weight $c \gamma_{1}$ or $c \gamma_{l}$ has all weight spaces of dimension 1 ; in particular, $\operatorname{dim}(M)=$ $(l+c) ! / l ! c !$.

Proof. This is 1.14 of [9].

We will occasionally use the Weyl character formula for dimensions of Weyl modules.

Finally, it was shown in [4] that when $X$ acts irreducibly on $W$, we may assume $W$ is in fact restricted as an $X$-module:

Lemma 2.6. If $X$ acts irreducibly on $W$, then as an $X$-module, $W$ has a restricted high weight.

Now let $P_{X}$ be a parabolic subgroup of $X$ and $P_{X}=Q_{X} L_{X}$ be a Levi decomposition with $T_{X} \leq L_{X}$ (if $P_{X}$ is $t$-stable, choose $L_{X}$ to also be $t$-stable). Now $X$ acts irreducibly on $W$ with high weight $\delta$, which is restricted by the Lemma above. We gave in [4] the following construction of a parabolic subgroup $P_{Y}$ of $Y$ (with $P_{Y}=Q_{Y} L_{Y}$ a Levi decomposition) such that $P_{X} \leq P_{Y}, Q_{X} \leq Q_{Y}, L_{X} \leq L_{Y}$. Let $Z=Z\left(L_{X}\right)^{\circ}$.

Lemma 2.7. The stabilizer in $Y$ of the commutator series

$$
W>\left[W, Q_{X}\right]>\left[W, Q_{X}, Q_{X}\right]>\cdots>0
$$

is a parabolic subgroup $P_{Y}$ of $Y$ satisfying the following:

1. $P_{X} \leq P_{Y}$ and $Q_{X} \leq Q_{Y}=R_{u}\left(P_{Y}\right)$.

2. $L_{Y}=C_{Y}(Z)$ is a Levi factor of $P_{Y}$ containing $L_{X}$.

3. If $T_{Y}$ is a maximal torus of $Y$ containing $T_{X}$, then $T_{Y} \leq L_{Y}$.

Proof. This is 2.7 of [4].

We give more information about this embedding for particular groups $X$ and parabolic subgroups $P_{X}$ below and in subsequent sections. For the next Lemma, we assume that $t \in G$ (where $t$ is the fixed outer automorphism of $X$ ) and $\left.V\right|_{X}=$ 
$V_{1} \oplus V_{2}$, with $V_{1}, V_{2}$ irreducible $X$-modules. This Lemma was proved in $[4,2.8$ and 2.9]:

Lemma 2.8. If $P_{X}$ is a t-stable parabolic subgroup of $X$ and $P_{X}$ is embedded in a parabolic subgroup $P_{Y}$ of $Y$ as above, then

1. $P_{Y}$ is likewise $t$-stable;

2. $V /\left[V, Q_{Y}\right]=V /\left[V, Q_{X}\right]=V_{1} /\left[V_{1}, Q_{X}\right] \oplus V_{2} /\left[V_{2}, Q_{X}\right]$ as $L_{X}$-modules.

Let $P_{Y}=L_{Y} Q_{Y}$ be a parabolic subgroup of $Y$. For each $\gamma \in \Pi(Y)-\Pi\left(L_{Y}^{\prime}\right)$, we define a certain normal subgroup $K_{Y}^{\gamma}$ of $P_{Y}$, as in [9, page 44]: Let $\Sigma_{\gamma}(Y)$ denote the set of roots in $\Sigma(Y)$ having $\gamma$-coefficient -1 and zero coefficient for other roots in $\Pi(Y)-\Pi\left(L_{Y}^{\prime}\right)$. Then let $K_{Y}^{\gamma}$ be the product of those $T_{Y}$-root subgroups $U_{\beta}$ for $\beta \in \Sigma^{-}(Y)-\Sigma^{-}\left(L_{Y}^{\prime}\right)-\Sigma_{\gamma}(Y)$. From the commutator relations it follows that $K_{Y}^{\gamma}$ is normal in $P_{Y}$ and we let $Q_{Y}^{\gamma}=Q_{Y} / K_{Y}^{\gamma}$. This construction also applies to a parabolic subgroup $P_{X}$ of $X$. In particular, if $P_{X}$ is a maximal parabolic subgroup corresponding to $\alpha \in \Pi(X)$, then set $Q_{X}^{\alpha}=Q_{X} / K^{\alpha}$, where $K^{\alpha}$ is the product of those $T_{X}$-root subgroups corresponding to roots having $\alpha$-coefficient strictly less than -1 .

The lemma below will be used heavily in dimension arguments.

Lemma 2.9. If $P_{X}=Q_{X} L_{X}$ is a maximal parabolic subgroup of $X$ corresponding to $\alpha \in \Pi(X)$ and $P_{X}$ is embedded in a parabolic subgroup $P_{Y}$ of $Y$ as in Lemma 2.7, then $\operatorname{dim}\left(V^{2}\left(Q_{Y}\right)\right) \leq \operatorname{dim}\left(Q_{X}^{\alpha}\right) \cdot \operatorname{dim}\left(V^{1}\left(Q_{X}\right)\right)$.

Proof. This is part of Proposition 2.14 in [9].

Lemma 2.10. If $P_{X}=Q_{X} L_{X}$ is a maximal parabolic subgroup corresponding to $\alpha \in \Pi(X)$, then

1. $K^{\alpha}=\left[Q_{X}, Q_{X}\right]$.

2. $Q_{X}^{\alpha}$ is an irreducible $L_{X}^{\prime}$-module with $-\alpha$ as its $T_{L_{X}^{\prime}}$-high weight.

Proof. See 3.2 in [9] (remembering that $X$ is of type $A_{m}, D_{m}$, or $E_{6}$ ).

Again assume $t \in G$. Let $P_{X}$ be a parabolic subgroup of $X$ (not necessarily $t$ stable) containing the fixed $t$-stable Borel subgroup $B_{X}$. Embed $P_{X}$ in a parabolic subgroup $P_{Y}$ of $Y$ via the above construction. Write $L_{Y}^{\prime}=L_{1} \times \cdots \times L_{r}$, a direct product of simple groups. By Lemma 2.2, $L_{Y}^{\prime}$ acts irreducibly on $V^{1}\left(Q_{Y}\right)=$ $V /\left[V, Q_{Y}\right]$. Then $V^{1}\left(Q_{Y}\right)=V^{1} \otimes \cdots \otimes V^{r}$ where for each $i, V^{i}$ is an irreducible module for $L_{i}$. The embedding $L_{X} \rightarrow L_{Y}$ gives an embedding of $L_{X}^{\prime}$ into $L_{1} \times$ $\cdots \times L_{r}$, and via the projections $L_{X}^{\prime} \rightarrow L_{i}$, any $L_{i}$-module, in particular $V^{i}$, can be regarded as a module for $L_{X}^{\prime}$.

Since $Q_{X} \leq Q_{Y}$, we have $\left[V, Q_{X}\right] \leq\left[V, Q_{Y}\right]$ and hence $V /\left[V, Q_{Y}\right]$ is a quotient of $V /\left[V, Q_{X}\right]=V_{1} /\left[V_{1}, Q_{X}\right] \oplus V_{2} /\left[V_{2}, Q_{X}\right]$, with each of these summands irreducible $L_{X}^{\prime}$-modules. Since $L_{X}^{\prime} \leq L_{Y}^{\prime}$, this implies that either $V /\left[V, Q_{Y}\right]$ is irreducible for $L_{X}^{\prime}$ or $V /\left[V, Q_{Y}\right]=V /\left[V, Q_{X}\right]=V_{1} /\left[V_{1}, Q_{X}\right] \oplus V_{2} /\left[V_{2}, Q_{X}\right]$. Lemma 2.8 tells us that the latter happens when $P_{X}$ is $t$-stable. The following was proved in [4, 2.11]:

Lemma 2.11. If $V, P_{X}=L_{X} Q_{X}, P_{Y}=L_{Y} Q_{Y}$, and $L_{i}$ are as above with $P_{X}$ $t$-stable, then only one $L_{i}$ acts nontrivially on $V /\left[V, Q_{Y}\right]$. 


\section{The CASE $X=A_{m}$}

As always, let $X<Y$ be simple algebraic groups over an algebraically closed field $K$ of characteristic $p$ ( $=0$ or a prime), with $X$ admitting an involutory graph automorphism $t$ which also acts on $Y$ and with $Y$ of classical type. Let $\left\{\beta_{i}\right\}$ $\left(\left\{\alpha_{i}\right\}=\Pi(Y)\right)$ be the set of simple roots for $X(Y)$ and let $\left\{\delta_{i}\right\} \quad\left(\left\{\lambda_{i}\right\}\right)$ be the corresponding fundamental dominant weights for $X(Y)$. The fixed $t$-stable Borel subgroup $B_{X}$ of $X$ contains a $t$-stable maximal torus $T_{X}$. Let $V=V(\lambda)$ be a restricted irreducible $t$-stable $Y$-module with high weight $\lambda=\sum a_{i} \lambda_{i}$, such that $\left.V\right|_{X\langle t\rangle}$ is irreducible, but $\left.V\right|_{X}=V_{1} \oplus V_{2}$, with $V_{1}, V_{2}$ restricted irreducible $X$ modules. We denote the $T_{X}$-high weight of $V_{1}$ by $b_{1} \delta_{1}+b_{2} \delta_{2}+\cdots+b_{m} \delta_{m}$, so the $T_{X}$-high weight of $V_{2}$ is $b_{m} \delta_{1}+b_{m-1} \delta_{2}+\cdots+b_{1} \delta_{m}$. The natural module for $Y$ is $W$.

The main result of the section is

Theorem 3.1. If $X$ acts irreducibly on $W$ with high weight $\delta, t$ acts on $W$, and $X$ is of type $A_{m}$, then $p \notin\{2,3,5,7\}, X=A_{3}, Y=D_{10}, \delta=2 \delta_{2}$, and the high weights of $\left.V\right|_{X},\left.V\right|_{Y}$ are as in $\mathrm{U}_{5}$ of Table 2.

Notice that since $t$ acts on $W$, the high weight $\delta=d_{1} \delta_{1}+\cdots+d_{m} \delta_{m}$ of $\left.W\right|_{X}$ must be symmetric, i.e. $d_{1}=d_{m}, d_{2}=d_{m-1}$, etc. But then by a result of Steinberg ([11, page 226]), $X$ fixes a nondegenerate bilinear form on $W$; the form is orthogonal if $p \neq 2$.

The strategy we use to rule out most possibilities for the high weight $\delta$ is to show that the construction (outlined in Lemma 2.7) of a parabolic subgroup of $Y$ containing the fixed ( $t$-stable) Borel subgroup of $X$ gives a contradiction in all but a few cases. After giving the Lemma which we usually use to produce the contradiction, we will treat the $A_{2}$ and $A_{3}$ cases first, followed by the general argument.

3.1. Some Facts About $P_{Y}$. We use the construction given in Lemma 2.7 of a parabolic subgroup $P_{Y}<Y$ containing the fixed $t$-stable Borel subgroup $B_{X}$. Namely, $P_{Y}$ is taken to be the stabilizer in $Y$ of the flag in $W$ given by " $U_{X}$-levels."

We want to use Lemma 3.2 below to produce a contradiction in most cases; we will show that $L_{Y}^{\prime}$ has a factor of type $A_{1}$ only under strong conditions. Before proceeding with the general proof, we need some facts about the flag in $W$ of which $P_{Y}$ is the stabilizer.

Recall that for $i \geq 0, W_{i}=\sum_{e_{1}+e_{2}+\cdots=i} W_{\delta-e_{1} \beta_{1}-e_{2} \beta_{2}-\ldots}$, the sum taken over $e_{j} \geq 0$. Each space $W_{i}$ is $T_{X}\langle t\rangle$-stable, and if $u \in U_{-\alpha}$, then

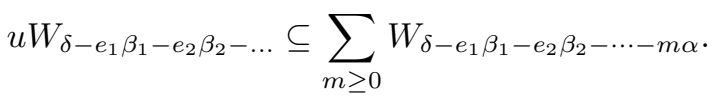

So $B_{X}\langle t\rangle$ stabilizes each factor

$$
\left(\sum_{i \geq m} W_{i}\right) /\left(\sum_{i \geq m+1} W_{i}\right) .
$$

By Lemma $2.4, W_{i} \cong\left[W, U_{X}^{i}\right] /\left[W, U_{X}^{i+1}\right]$.

Let $l$ be minimal with respect to $\left[W, U_{X}^{l+1}\right]=0$, and notice that $l$ is then the level of the low weight $-\delta$. If $Y=\mathrm{Sp}(W)$ or $Y=\mathrm{SO}(W)$, with the form denoted by $($,$) , then we noted in \left[4\right.$, proof of 2.7] that $(u, v)=0$ for $u \in W_{i}, v \in W_{j}$ unless 
$i+j \leq l$. Thus the $W_{i}$ for $i>l / 2$, along with a maximal totally singular subspace of $W_{l / 2}$ (if $l$ is even), span a maximal totally singular subspace of $W$.

Let $w^{+}$be an $T_{X}$-high weight vector of $W$. Then $W_{0}=\left\langle w^{+}\right\rangle, W_{l}=\left\langle w_{0} w^{+}\right\rangle$, and $B_{X}$ is contained in the full stabilizer $P_{Y}$ of the flag

$$
W=\sum_{i \geq 0} W_{i} \geq \sum_{i \geq 1} W_{i} \geq \cdots \geq \sum_{i \geq l} W_{i}=\left\langle w_{0} w^{+}\right\rangle \geq 0 .
$$

Let $P_{Y}=L_{Y} Q_{Y}$ be a Levi decomposition of $P_{Y}$; then if $u \in U_{X}, w \in W_{m}$, we have $u w-w \in \sum_{i \geq m+1} W_{i}$, so $U_{X} \leq Q_{Y}$. We have $T_{X} \leq L_{Y}=C_{Y}(Z)$ for $Z=Z\left(L_{X}\right)^{\circ}$.

Choose a basis for each $W_{i}$ (with the basis for $W_{l / 2}$ chosen maximally hyperbolic — note that $W_{l / 2} \cong\left(\sum_{i \geq l / 2} W_{i}\right) /\left(\sum_{i>l / 2} W_{i}\right)$ is the only possible non-singular quotient in the flag); the union of these bases is a basis for $W$. With respect to this basis, $L_{Y}^{\prime}$ consists of block matrices, each block corresponding to $W_{i}$ for some $i$. On the other hand, each $W_{i}$ for $0<i \leq l / 2$ corresponds to a connected component of the Dynkin diagram for $L_{Y}$. So the only possibilities for an $A_{1}$ to appear as one of the simple factors of $L_{Y}^{\prime}$ are when $\operatorname{dim}\left(W_{i}\right)=2$ for some $i<l / 2$ or $\operatorname{dim}\left(W_{l / 2}\right) \leq 4$.

To show $\operatorname{dim}\left(W_{i}\right) \geq m$, it suffices to find $m T_{X}$-weights of $W$ which occur in $W_{i}$. By the result in [12], weights which appear in characteristic 0 also appear in characteristic $p$. This is the approach we use to obtain contradictions for most embeddings $X \hookrightarrow Y$.

For a $T_{X}$-weight $\omega$ of $W, \omega+\omega^{t}=\omega-w_{0} \omega$ is a sum of roots, and we let $l_{\omega}$ be the height of $\omega+\omega^{t}$ in the root lattice (the number of summands when we express $\omega+\omega^{t}$ as a sum of fundamental roots). So for $l$ as above, $l=l_{\delta}$ where $\delta$ is the $T_{X}$-high weight of $W$.

The constructions in this section also apply to the embedding of an arbitrary parabolic subgroup $P_{X}=Q_{X} L_{X}$ of $X$ in a parabolic subgroup $P_{Y}$ of $Y$, using $Q_{X}$-levels in place of the $U_{X}$-levels. The weights appearing in $W_{i}$ then are those of the form $\delta-e_{1} \beta_{1}-\cdots-e_{m} \beta_{m}$ where the sum of the $e_{j}$ for $\beta_{j} \in \Pi(X)-\Pi\left(L_{X}^{\prime}\right)$ is $i$. Again by Lemma $2.4, W_{i} \cong\left[W, Q_{X}^{i}\right] /\left[W, Q_{X}^{i+1}\right]$.

One more fact: If $P_{X}$ is a $t$-stable parabolic subgroup of $X$ (including $P_{X}=B_{X}$ ), then each $W_{i}$ is clearly $t$-stable (since then $W_{\delta-e_{1} \beta_{1}-e_{2} \beta_{2}-\cdots-e_{m} \beta_{m}}$ is sent by $t$ to $\left.W_{\delta-e_{m} \beta_{1}-e_{m-1} \beta_{2}-\cdots-e_{1} \beta_{m}}\right)$. So $P_{Y}$ is $t$-stable. But then $\left[V, Q_{Y}\right]$ is $T_{X}\langle t\rangle$-stable, hence $\left[V, Q_{Y}\right]=\left[V, Q_{X}\right]\left(\left[V, Q_{X}\right] \leq\left[V, Q_{Y}\right]\right.$ since $Q_{X} \leq Q_{Y}$, and we get equality because $V /\left[V, Q_{X}\right]$ is an irreducible $T_{X}\langle t\rangle$-module and $\left.T_{X} \leq L_{Y}\right)$. So if $P_{X}$ is $t$-stable, then $V /\left[V, Q_{Y}\right]$ is a sum of two irreducible $L_{X}^{\prime}$-modules.

The following lemma provides the basis for the proof of the section's main result; it will also be used throughout the paper.

Lemma 3.2. If $P_{Y}$ is a t-stable parabolic subgroup of $Y$ such that $B_{X}<P_{Y}, U_{X}<$ $Q_{Y}, T_{X}<L_{Y}^{\prime}$ (where $P_{Y}=Q_{Y} L_{Y}, B_{X}=U_{X} T_{X}$ are the Levi decompositions), then at least one of the simple factors of $L_{Y}^{\prime}$ has type $A_{1}$; and if this factor corresponds to $\alpha_{j}$, then $a_{j}=1$. In addition, $a_{i}=0$ for $\alpha_{i} \in \Pi\left(L_{Y}^{\prime}\right), i \neq j$.

Proof. This is Lemma 5.2 of [4].

3.2. The Cases $X=A_{2}$ and $X=A_{3}$. We use Lemma 3.2 heavily. As always, $\delta=d_{1} \delta_{1}+d_{2} \delta_{2}+\cdots$ is the $T_{X}$-high weight of $W$. Let $\beta$ be a nonnegative sum of fundamental roots of $X$, of height $j$ in the root lattice. Note that if $\delta-\beta$ is a dominant weight such that the $X$-module with high weight $\delta-\beta$ has $\geq m$ weights at level $i$, then $W$ has $\geq m$ weights at level $j+i$. So in our attempt to prove that 
there cannot be a $U_{X}$-level of dimension 2 in $W$, we will proceed by induction on the high weight $\delta$.

$X=A_{2}$. Since $\delta$ is symmetric, $\delta=a \delta_{1}+a \delta_{2}$ for some $a>0$. Here we will always have $\operatorname{dim}\left(W_{1}\right)=2$, since the only two weight spaces in level 1 are $\delta-\beta_{1}$ and $\delta-\beta_{2}$, both of dimension 1; we will deal with level 1 after we discuss levels 2 and higher. In evaluating the numbers of weights at these levels, we will first use an induction to deal with the case $a \geq 4$, and then deal with $a=3$ and $a=2$.

Assume $a=4$. Then $l=l_{\delta}=16$, so we must check to level 8 (we must list three weights at every level 2-7, and 5 at level 8 ). We have the weights in the table below:

$$
\begin{array}{cl}
\text { Level } & \text { Weights } \\
2 & \delta-2 \beta_{1}, \delta-\beta_{1}-\beta_{2}, \delta-2 \beta_{2} \\
3 & \delta-3 \beta_{1}, \delta-2 \beta_{1}-\beta_{2}, \delta-3 \beta_{2} \\
4 & \delta-4 \beta_{1}, \delta-3 \beta_{1}-\beta_{2}, \delta-4 \beta_{2} \\
5 & \delta-4 \beta_{1}-\beta_{2}, \delta-3 \beta_{1}-2 \beta_{2}, \delta-2 \beta_{1}-3 \beta_{2} \\
6 & \delta-4 \beta_{1}-2 \beta_{2}, \delta-3 \beta_{1}-3 \beta_{2}, \delta-2 \beta_{2}-4 \beta_{2} \\
7 & \delta-5 \beta_{1}-\beta_{2}, \delta-4 \beta_{1}-3 \beta_{2}, \delta-3 \beta_{1}-4 \beta_{2} \\
8 & \delta-6 \beta_{1}-2 \beta_{2}, \delta-5 \beta_{1}-3 \beta_{2}, \delta-4 \beta_{1}-4 \beta_{2}, \delta-3 \beta_{1}-5 \beta_{2}, \delta-2 \beta_{1}-6 \beta_{2} .
\end{array}
$$

So $L_{Y}^{\prime}$ has no factors of type $A_{1}$ except possibly the factor $L_{1}$ corresponding to $W_{1}$.

Assume $a>4$. Then $\delta-\beta_{1}-\beta_{2}=(a-1) \delta_{1}+(a-1) \delta_{2}$ is dominant, and by induction $\delta$ has enough weights at all levels except possibly at levels 1,2 and 3 . At level 2 , we have $\delta-2 \beta_{1}, \delta-\beta_{1}-\beta_{2}$, and $\delta-2 \beta_{2}$; at level $3, \delta-3 \beta_{1}, \delta-2 \beta_{1}-\beta_{2}$, and $\delta-3 \beta_{2}$. So again, $L_{1}$ is the only possible $A_{1}$-factor of $L_{Y}^{\prime}$.

Assume $a=3$. Then $l=12$; we must check dimensions to level 6 . In levels 2-5, we have enough weights as above. So we must show that $W_{6}$ has dimension at least 5 . The weights at level 6 are $\delta-4 \beta_{1}-2 \beta_{2}, \delta-3 \beta_{1}-3 \beta_{2}$, and $\delta-2 \beta_{1}-4 \beta_{2}$. If $p \neq 7$, then $\operatorname{dim}\left(W_{\delta-3 \beta_{1}-3 \beta_{2}}\right) \geq 3$, so $\operatorname{dim}\left(W_{6}\right) \geq 5$. So unless $p=7$, here again we have only the $L_{1}=A_{1}$ possibility.

If $a=2$, then $l=8$ and we must check dimensions to level 4 . For level 2, we have enough weights as above. At level 3 , we have $\delta-2 \beta_{1}-\beta_{2}$ and $\delta-\beta_{1}-2 \beta_{2}$; if $p \neq 5$, each has dimension 2 , so $\operatorname{dim}\left(W_{3}\right)>3$. At level 4 , the weights are $\delta-3 \beta_{1}-\beta_{2}$, $\delta-2 \beta_{1}-2 \beta_{2}$, and $\delta-\beta_{1}-3 \beta_{2}$. If $p \neq 5$, then $\delta-2 \beta_{1}-2 \beta_{2}$ has dimension $\geq 3$, so $\operatorname{dim}\left(W_{4}\right) \geq 5$. As above, unless $p=5$, we have only the $L_{1}=A_{1}$ possibility.

From the construction of $P_{Y}$ we can see that in any case covered above (including $(a, p)=(2,5),(3,7))$, there is only one node in the Dynkin diagram between the $L_{i}$, since there are no $U_{X^{-}}$-levels of dimension 1 other than $\left\langle w^{+}\right\rangle$. Also notice that from the $a=2$ and $a=3$ cases above we know the embeddings in the cases $(a, p)=(2,5),(3,7)$ (we simply compute the dimensions of the levels): If $a=2, p=5$, then $\operatorname{dim}(W)=19$, so $Y$ has type $B_{9}$ and $P_{Y}$ is the parabolic subgroup of $Y$ corresponding to the indicated nodes

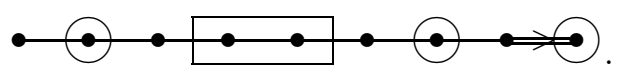

If $a=3, p=7$ then $\operatorname{dim}(W)=37, Y$ is of type $B_{18}$, and $P_{Y}$ corresponds to

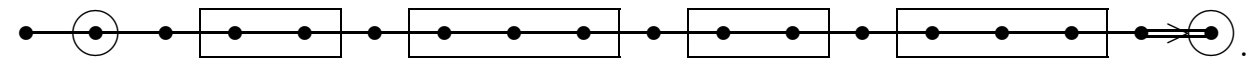

So the possibilities are 1) $a_{2}=1$, with the first simple factor of $L_{Y}^{\prime}$ corresponding to $\bullet \cdots$; 2 ) $a=2, p=5$; 3) $a=3, p=7$; 4) $a=1$. By Lemma 3.2 , in the 
marking for the high weight of $V$ on the Dynkin diagram for $Y$ there is only one non-zero label on the nodes representing $L_{Y}$, and this non-zero label must be a 1 on a node corresponding to an $A_{1}$ factor of $L_{Y}^{\prime}$; call this node $\gamma$. By the comment above, all nodes in the Dynkin diagram are either in or adjacent to $\Pi\left(L_{Y}^{\prime}\right)$ (except possibly in case 4). Our aim is to show that all nodes except $\gamma$ have marking 0 .

As in the introduction, let $V^{2}\left(Q_{Y}\right)=\left[V, Q_{Y}\right] /\left[V, Q_{Y}, Q_{Y}\right]$; similarly define $V^{2}\left(U_{X}\right)$ and $V_{i}^{2}\left(U_{X}\right)$ for $i=1,2$. Recall that $\lambda$ is the $T_{Y}$-high weight of $V$. By Lemma 2.4, the weights in $V^{2}\left(Q_{Y}\right)$ are those of the form $\left.(\lambda-\beta)\right|_{T_{L_{Y}}}$ where $\beta \in \Sigma^{+}(Y)$ and there exists $\epsilon \in \Pi(Y)-\Pi\left(L_{Y}\right)$ such that $\beta-\epsilon \in \Sigma\left(L_{Y}\right)$. Then by Lemma 2.4, we have $\operatorname{dim}\left(V^{2}\left(Q_{Y}\right)\right) \leq \operatorname{dim}\left(V^{2}\left(U_{X}\right)\right) \leq 4$.

First consider the case $a=1$. If $p \neq 3$, then $\operatorname{dim}(W)=8$ and $Y=D_{4}$; if $p=3$, then $\operatorname{dim}(W)=7$ and $Y=B_{3}$. In both cases, constructing $P_{Y}$ as usual, we have $\alpha_{2} \in \Pi\left(L_{Y}^{\prime}\right)$, so the only possibility for an $A_{1}$ factor of $L_{Y}$ to occur is $\Pi\left(L_{Y}^{\prime}\right)=\left\{\alpha_{2}\right\}$ (as all other nodes adjoin $\alpha_{2}$ ). For $p \neq 3$, if another node has a non-zero label, say $\alpha_{3}$ (all are equivalent by symmetry for this argument), then in $V^{2}\left(Q_{Y}\right)$ we have the high weights $\lambda-\left.\alpha_{3}\right|_{T_{L_{Y}^{\prime}}}$, giving a composition factor of dimension 3 and $\lambda-\alpha_{2}-\alpha_{1}$ and $\lambda-\alpha_{2}-\alpha_{4}$ each giving one of dimension 1 . But this contradicts $\operatorname{dim}\left(V^{2}\left(Q_{Y}\right)\right) \leq 4$. So $\lambda=\lambda_{2}$, giving an example of 1) above, which we deal with below. If $p=3$ and $a_{1}, a_{3} \neq 0$, then $\lambda-\left.\alpha_{1}\right|_{T_{L_{Y}^{\prime}}}, \lambda-\left.\alpha_{3}\right|_{T_{L_{Y}^{\prime}}}$ each give a composition factor of dimension 3 in $V^{2}\left(Q_{Y}\right)$, again a contradiction. Finally, irreducible $B_{3}$-modules with high weights $e \lambda_{1}+\lambda_{2}$ or $\lambda_{2}+e \lambda_{3}$ are all too large to be the sum of two restricted irreducible $A_{2}$-modules unless $e=0$ (by counting weights that appear in the Weyl module of $\mathcal{W}_{B_{3}}\left(e \lambda_{1}+\lambda_{2}\right)$ or $\mathcal{W}_{B_{3}}\left(\lambda_{2}+e \lambda_{3}\right)$; all these weights appear in $V$ by [8]). So again $\lambda=\lambda_{2}$, which we deal with below.

For cases 1)-3), assume there is an $\epsilon \in \Pi(Y)$ which adjoins $\gamma$ and has non-zero marking $m$. If $\epsilon$ is not an end node, then it also adjoins another factor $L_{l}$ of $L_{Y}^{\prime}$ by our comment above that there are never two adjacent nodes outside $\Pi\left(L_{Y}^{\prime}\right)$; then $\lambda-\epsilon$ is an $L_{Y}^{\prime}$-high weight in $V^{2}\left(Q_{Y}\right)$, and the $L_{Y}^{\prime}$-high weight module of this high weight has dimension $\geq 6\left(\left.(\lambda-\epsilon)\right|_{T_{L_{Y}^{\prime}}}=2 \lambda_{j}+\lambda_{k}\right.$ where $\lambda_{j}$ is the fundamental dominant weight corresponding to $\gamma$ and $\lambda_{k}$ is the node of $L_{l}$ which adjoins $\epsilon$ - the $L_{Y}^{\prime}$-module with this high weight has dimension at least $2 \cdot 3=6$ ). This contradicts $\operatorname{dim}\left(V^{2}\left(Q_{Y}\right)\right) \leq 4$.

If $\epsilon$ is an end node, it cannot be the short root in a $B_{n}$ (that root is in $\Pi\left(L_{Y}^{\prime}\right)$ because we saw that $\operatorname{dim}\left(W_{l / 2}\right) \geq 3$ in all cases). Then we have the picture

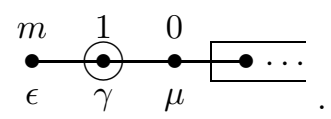

We have high weights $\left.(\lambda-\epsilon)\right|_{T_{L_{Y}^{\prime}}}$ of dimension 3 and $\left.(\lambda-\gamma-\mu)\right|_{T_{L_{Y}^{\prime}}}$ of dimension $\geq 2$. Again, this contradicts $\operatorname{dim}\left(V^{2}\left(Q_{Y}\right)\right) \leq 4$. So all nodes adjoining $\gamma$ have 0 label.

Assume there is an $\epsilon \in \Pi(Y)$ which does not adjoin $\gamma$ and has non-zero marking $m$. By Lemma 3.2, $\epsilon \notin \Pi\left(L_{Y}^{\prime}\right)$, and $\epsilon$ adjoins $\Pi\left(L_{Y}^{\prime}\right)$ since every fundamental root is either in $\Pi\left(L_{Y}^{\prime}\right)$ or adjoins it. If $\gamma$ is not an end node, we have the pictures (different pieces of the Dynkin diagram for $Y$ )

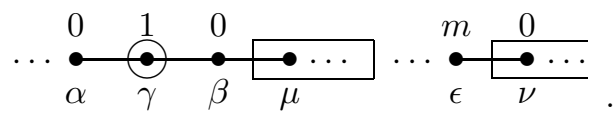


Here $\lambda-\alpha-\left.\gamma\right|_{T_{L_{Y}^{\prime}}}$ is a high weight in $V^{2}\left(Q_{Y}\right)$, giving dimension $\geq 1 ; \lambda-\gamma-\left.\beta\right|_{T_{L_{Y}^{\prime}}}$ gives dimension $\geq 2$; and $\lambda-\epsilon$ gives dimension $\geq 2$. Again, this is a contradiction. The node $\gamma$ can be an end node only in the cases $(a, p)=(2,5),(3,7)$ (these are the only cases in which an $A_{1}$ factor of $L_{Y}^{\prime}$ corresponds to an end node of the Dynkin diagram for $Y$ ), with $\gamma$ the short root of a $B_{n}$; then the picture is

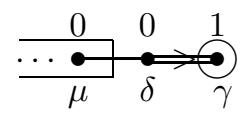

Here $\lambda-\gamma-\left.\delta\right|_{T_{L_{Y}^{\prime}}}$ gives dimension $\geq 4$; and $\lambda-\left.\epsilon\right|_{T_{L_{Y}^{\prime}}}$ gives dimension $\geq 1$, again a contradiction. So all nodes other than $\gamma$ must have label 0 .

For both of the above non-end node cases, similar arguments hold if there is a double bond in one of the relevant pieces of the Dynkin diagram for $Y$.

So $\gamma$ is the only node in the Dynkin diagram with a non-zero label. We need only to show that the few remaining possibilities do not lead to examples.

Case 1): $V$ has high weight $\lambda_{2}$. Then $V \cong \bigwedge^{2} W$ by [7, II.2.15]. Regard $W$ as an $X$-module. Remember that $\delta=a \delta_{1}+a \delta_{2}$ is the $T_{X}$-high weight for $W$. Let $v_{1} \in W_{\delta}$ be a maximal vector in $W ; 0 \neq v_{2} \in W_{\delta-\beta_{1}}, 0 \neq v_{3} \in W_{\delta-\beta_{2}}$. Then $v_{1} \wedge v_{2}$ and $v_{1} \wedge v_{3}$ are $X$-maximal vectors in $V$, so $K X\left(v_{1} \wedge v_{2}\right) \oplus K X\left(v_{1} \wedge v_{3}\right)=V$.

We now consider the dimension of $K X\left(v_{1} \wedge v_{2}\right)$. The vector $v_{1} \wedge v_{2}$ has weight $2 \delta-\beta_{1}=(2 a-2) \delta_{1}+(2 a+1) \delta_{2}$. So $\operatorname{dim}\left(K X\left(v_{1} \wedge v_{2}\right)\right) \leq \operatorname{dim}($ Weyl module $)=$ $\frac{1}{2}(2 a-1)(2 a+2)(4 a+1)=8 a^{3}+6 a^{2}-3 a-1$. Also, $\operatorname{dim}\left(K X\left(v_{1} \wedge v_{3}\right)\right)=$ $\operatorname{dim}\left(K X\left(v_{1} \wedge v_{2}\right)\right)$ (since $t$ interchanges them), so $\operatorname{dim}(V) \leq 16 a^{3}+12 a^{2}-6 a-2$.

On the other hand, $\operatorname{dim}(W) \geq 3 a^{2}+3 a+1$ (this is the number of weights that appear in the Weyl module with the same $T_{X}$-high weight as $W$; all these weights appear in $W)$, and $\operatorname{dim}(V)=\operatorname{dim}\left(\bigwedge^{2} W\right)=\left(\begin{array}{c}\operatorname{dim}(W) \\ 2\end{array}\right) \geq\left(\begin{array}{c}3 a^{2}+3 a+1 \\ 2\end{array}\right)=$ $\left(3 a^{2}+3 a+1\right)\left(3 a^{2}+3 a\right) / 2=\left(9 a^{4}+18 a^{3}+12 a^{2}+3 a\right) / 2$. So $9 a^{4}+18 a^{3}+12 a^{2}+3 a \leq$ $2\left(16 a^{3}+12 a^{2}-6 a-2\right)$. But this has no solutions in positive integers. So this case is ruled out.

The only cases not ruled out by the above now follow.

Case 2): $(a, p)=(2,5)$ (here remember $\left.Y=B_{9}\right)$. If $\lambda=\lambda_{7}$ then the picture is

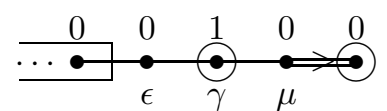

and $\lambda-\gamma-\left.\epsilon\right|_{L_{Y}}, \lambda-\gamma-\left.\mu\right|_{L_{Y}}$ each give dimension $\geq 3$. So $\lambda \neq \lambda_{7}$. If $\lambda=\lambda_{9}$, then $\operatorname{dim}(V)=2^{9}=512$. But the dimension of any irreducible $A_{2}$-module with high weight $c \delta_{1}+d \delta_{2}(c, d<5, c \neq d)$ is at most 90 . So $V$ is too large to be the sum of two restricted irreducible modules for $X$.

Case 3): $(a, p)=(3,7)$ (here $\left.Y=B_{18}\right)$ with $\lambda=\lambda_{18}$. Here $\operatorname{dim}(V)=2^{18}$. But $c, d<7, c \neq d$; again, $V$ is too large to be the sum of two restricted irreducible $X$-modules.

So $X$ is not of type $A_{2}$.

$X=A_{3}$. We use a similar induction. Let $\delta=a \delta_{1}+b \delta_{2}+a \delta_{3}$ be the $T_{X}$-high weight of $W$. First we eliminate the case $b=0$ with the $A_{1}$ factor of $L_{Y}^{\prime}$ (referred to in Lemma 3.2) corresponding to $\alpha_{2}$.

Assume $\delta=a \delta_{1}+a \delta_{3}$ and $\lambda=\lambda_{2}+\cdots$. Let $P_{X}=L_{X} Q_{X}$ be the maximal parabolic subgroup of $X$ corresponding to $\beta_{3} \in \Pi(X)$, and embed $P_{X}$ in a parabolic 
subgroup $P_{Y}$ of $Y$ via the construction in Lemma 2.7. Now $L_{1}$ (the simple factor of $L_{Y}^{\prime}$ corresponding to $Q_{X}$-level 0 of $\left.W\right)$ is of type $A_{2}$ if $a=1$ and type $A_{l}$ with $l \geq 5$ if $a>1$, and the root system of $L_{1}$ contains $\alpha_{1}$. (We determine the rank of $L_{1}$ by counting weights that appear in $Q_{X}$-level 0 of $W$, using Suprunenko's result [12] that weights which appear in the Weyl module also appear in the irreducible module.)

Assume $V /\left[V, Q_{Y}\right]$ is reducible as an $L_{X}$-module; that is,

$$
V /\left[V, Q_{Y}\right]={ }_{L_{X}} V_{1} /\left[V_{1}, Q_{X}\right] \oplus V_{2} /\left[V_{2}, Q_{X}\right] .
$$

Then $Z \leq Z\left(L_{Y}\right)$ since $L_{Y}=C_{Y}(Z)$. So $Z$ induces scalars on $V /\left[V, Q_{Y}\right]$ (an irreducible $L_{Y}$-module):

$$
\begin{aligned}
Z & =\left\{\left(\begin{array}{cccc}
c & 0 & 0 & 0 \\
0 & c & 0 & 0 \\
0 & 0 & c & 0 \\
0 & 0 & 0 & c^{-3}
\end{array}\right) \mid c \in K^{*}\right\} \\
& =\left\{h_{\beta_{1}}(c) h_{\beta_{2}}\left(c^{2}\right) h_{\beta_{3}}\left(c^{3}\right) \mid c \in K^{*}\right\} .
\end{aligned}
$$

Now $h_{\beta_{1}}(c) h_{\beta_{2}}\left(c^{2}\right) h_{\beta_{3}}\left(c^{3}\right)$ acts as multiplication by $c^{b_{1}+2 b_{2}+3 b_{3}}$ on a high weight vector $v_{1} \in V_{1}$ and as multiplication by $c^{b_{3}+2 b_{2}+3 b_{1}}$ on a high weight vector $v_{2} \in V_{2}$. But by our assumption, $v_{1}$ and $v_{2}$ both have nonzero images in $V /\left[V, Q_{Y}\right]$; because $b_{1}+2 b_{2}+3 b_{3} \neq b_{3}+2 b_{2}+3 b_{1}$, we have a contradiction $\left(b_{1} \neq b_{3}\right.$ because $\left.V_{1} \varlimsup_{X} V_{2}\right)$.

So $V /\left[V, Q_{Y}\right]$ is an irreducible $L_{X}$-module and thus an irreducible $L_{X}^{\prime}$-module by Lemma 2.2. Assume $V /\left[V, Q_{Y}\right]={ }_{X} V_{1} /\left[V_{1}, Q_{X}\right]$.

Now we are in the situation studied in $[9]: V /\left[V, Q_{Y}\right]$ is an irreducible module for $L_{X}^{\prime}$ (of type $A_{2}$ ) and for $L_{1}$. As there are no examples matching this setup in [9, Table 1], we know that either the embedding $L_{X}^{\prime} \hookrightarrow L_{1}$ is an isomorphism or $V /\left[V, Q_{Y}\right]$ is the natural module for $L_{1}$. Both possibilities are excluded if $a>1$, as then $L_{1}$ is of type $A_{l}$ with $l \geq 5$ (so $L_{1} \neq L_{X}^{\prime}$ ), and $a_{2}=1$ (so $V /\left[V, Q_{Y}\right]$ is not the natural module for $L_{1}$ ). On the other hand, if $a=1$, then necessarily $L_{X}^{\prime} \cong L_{1}$ (as $W /\left[W, Q_{Y}\right]$ then has $L_{X}^{\prime}$-high weight $\delta_{1}$ ).

So $V /\left[V, Q_{Y}\right]$ must have $L_{X}^{\prime}$-high weight $a_{1} \delta_{1}+\delta_{2}$, since it has $L_{1}$-high weight $a_{1} \lambda_{1}+\lambda_{2}$ and the embedding is an isomorphism. But $V_{1} /\left[V_{1}, Q_{X}\right]$ also has high weight $b_{1} \delta_{1}+b_{2} \delta_{2}$. So $a_{1}=b_{1}$ and $b_{2}=1$.

Now the argument above can be repeated with the maximal parabolic subgroup of $X$ corresponding to $\beta_{1}$ instead of $\beta_{3}$, with the conclusion that $b_{3}=a_{1}$. But then $b_{1}=b_{3}$, a contradiction.

So if $\delta=a \delta_{1}+a \delta_{3}$, then $a_{2}=0$.

Every weight of the form $a \delta_{1}+b \delta_{2}+a \delta_{3}$ except $\delta_{1}+\delta_{3}, \delta_{2}$, and $2 \delta_{2}$ has one of $2 \delta_{1}+2 \delta_{3}$ or $\delta_{1}+\delta_{2}+\delta_{3}$ as a subdominant weight. It is easy to check, as in the $A_{2}$ case, that the modules with these latter two high weights have enough weights at every level, so we can proceed by induction: If $b<2$ and $a>2$, then by induction $\delta-\beta_{1}-\beta_{2}-\beta_{3}=(a-1) \delta_{1}+b \delta_{2}+(a-1) \delta_{3}$ has enough weights at all levels; we need to check $\delta$-levels 2-5. As before, there are enough weights in each of these levels, so by induction, $L_{1}$ is the only possible factor of $L_{Y}^{\prime}$ of type $A_{1}$.

If $b \geq 2$, then by induction $\delta-\beta_{2}$ has enough weights at all levels, and we need to check only $\delta$-levels 1 and 2. If $a=0$, then $\operatorname{dim}\left(W_{1}\right)=1$, so $L_{1}$ is trivial; if $a>0$, then $\operatorname{dim}\left(W_{1}\right)=3$, so $L_{1}$ is of type $A_{2}$. Again, there are enough weights at level 2 . So there are no possible $A_{1}$ factors of $L_{Y}^{\prime}$ here. 
So the possibilities which have not been ruled out are $\delta=\delta_{1}+\delta_{3}, \delta=\delta_{2}$, and $\delta=2 \delta_{2}$. If $\delta=\delta_{2}$, then $X=S O(W)=Y$, so there are no examples here. If $\delta=\delta_{1}+\delta_{3}$, we can check the dimensions of the weight spaces and find that $\operatorname{dim}(W)=15$ if $p \neq 2$, and $P_{Y}$ is the parabolic subgroup of $Y$ corresponding to the subset $\left\{\alpha_{2}, \alpha_{4}, \alpha_{5}, \alpha_{7}\right\}$ of $\Pi(Y)$, as indicated on this picture of the Dynkin diagram

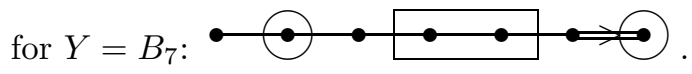

If $p=2$, then $\operatorname{dim}(W)=14$, and in [9, page 273] it is shown that $X$ stabilizes a quadratic form on $W$. So in this case $Y=D_{7}$ and counting dimensions of $U_{X}$-levels,

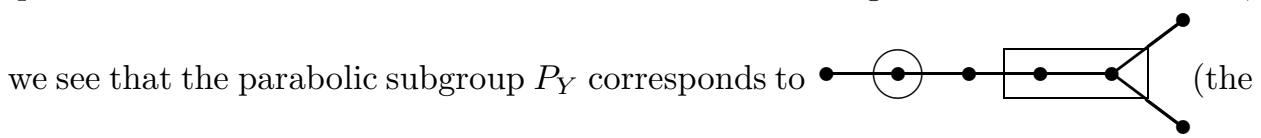
stabilizer of the 2-dimensional level 3 is $\mathrm{SO}_{2}$, which is a torus). So Lemma 3.5 rules out this case.

If $\delta=2 \delta_{2}$, again we can check dimensions of all the weight spaces to find $\operatorname{dim}(W)=20(p \neq 2,3), \operatorname{dim}(W)=19(p=3)$, and $P_{Y}$ is indicated by the circled nodes of the Dynkin diagram for $Y$,

or
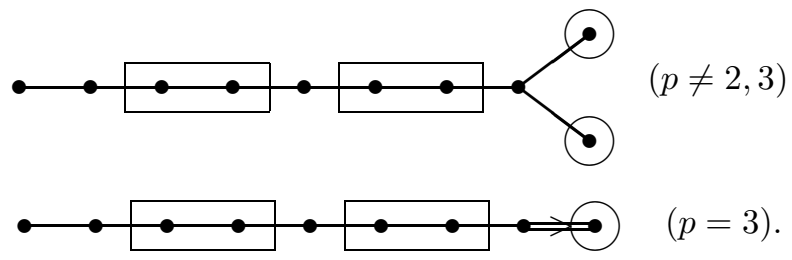

Notice we need not consider $p=2$ by Lemma 2.6.

So the cases we must deal with are

$$
\left.W\right|_{X} \quad Y \text { and embedding of } B_{X}<P_{Y}
$$
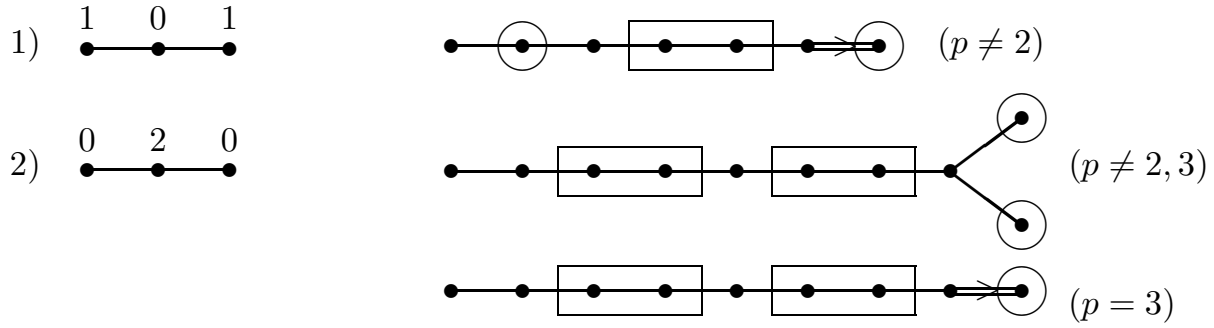

In the marking for $V$ on $\Pi(Y)$, there is a 1 on one of the nodes $\alpha_{j}$ corresponding to an $A_{1}$ factor of $L_{Y}^{\prime}$. There can be no other nonzero marking on any of the indicated nodes, since $\operatorname{dim}\left(V /\left[V, Q_{Y}\right]\right)=2$. Recall that $j \neq 2$ by Lemma 3.5 ; thus, as we see from the pictures above, $j=n$ is the only possibility (or $j=n-1$ in case 2 , but we may assume $j=n$ by symmetry). We claim that $\alpha_{n}$ is the only node with a nonzero label.

Claim 3.3. In any of the above cases $\lambda=\lambda_{n}$.

Proof. Since $U_{X} \leq Q_{Y}$, we have $\left[V, Q_{Y}, Q_{Y}\right] \geq\left[V, U_{X}, U_{X}\right]$. Then because $\left[V, Q_{Y}\right]$ $=\left[V, U_{X}\right]$, we have $\operatorname{dim}\left(\left[V, Q_{Y}\right] /\left[V, Q_{Y}, Q_{Y}\right]\right) \leq \operatorname{dim}\left(\left[V, U_{X}\right] /\left[V, U_{X}, U_{X}\right]\right) \leq 6$ by Lemma 2.4. The weights that appear in $V^{2}\left(Q_{Y}\right)=\left[V, Q_{Y}\right] /\left[V, Q_{Y}, Q_{Y}\right]$ are those of the form $\lambda-\beta$, where if $\beta=\sum e_{i} \alpha_{i}$, then the sum of the $e_{i}$ for those 
$\alpha_{i} \in \Pi(Y)-\Pi\left(L_{Y}^{\prime}\right)$ is 1 . Let us consider the above cases. Remember $\lambda=\sum a_{i} \lambda_{i}$ is the $T_{Y}$-high weight for $V$.

1) The node $\alpha_{7}$ has label $1\left(a_{7}=1\right)$. Consider the possibilities for another nonzero label. If $a_{1} \neq 0$, then $\lambda-\alpha_{1}$ is a high weight in $V^{2}\left(Q_{Y}\right)$, giving a composition factor of dimension 4 . Another high weight is $\lambda-\alpha_{6}-\alpha_{7}$, giving dimension 6 since $p \neq 2$. But above we noted that $\operatorname{dim}\left(V^{2}\left(Q_{Y}\right)\right) \leq 6$. So $a_{1}=0$. If $a_{3} \neq 0$, then $\lambda-\alpha_{3}$ gives $\operatorname{dim}\left(V^{2}\left(Q_{Y}\right)\right) \geq 12$; so $a_{3}=0$. If $a_{6} \neq 0$, then $\lambda-\alpha_{6}$ gives dimension 12 since $p \neq 2$. So $a_{6}=0$.

2) If $p \neq 3$, we are in the case $Y=D_{10}$, with $a_{10}=1$. If $a_{8} \neq 0$, then $\lambda-\alpha_{8}$ gives dimension 18 in $V^{2}\left(Q_{Y}\right)$. If $a_{5} \neq 0$, then $\lambda-\alpha_{5}$ gives dimension 18. If $a_{2} \neq 0$, then $\lambda-\alpha_{2}$ gives dimension 6 and $\lambda-\alpha_{8}-\alpha_{9}$ gives dimension 6 . If $a_{1} \neq 0$, then $\lambda-\alpha_{8}-\alpha_{9}$ gives dimension 6 and $\lambda-\alpha_{1}$ dimension 2. So in fact $a_{i}=0$ for all $i \neq 9$.

If $p=3$, then $Y=B_{9}$ and $a_{9}=1$. If $a_{8} \neq 0$, then $\lambda-\alpha_{8}$ gives dimension 6 and $\lambda-\alpha_{8}-\alpha_{9}$ gives dimension $\geq 1$; so $a_{8}=0$. If $a_{5} \neq 0, \lambda-\alpha_{5}$ gives dimension 18; if $a_{2} \neq 0, l-\alpha_{2}$ gives dimension 6 and $\lambda-\alpha_{8}-\alpha_{9}$ gives dimension 6 . If $a_{1} \neq 0$, then $\lambda-\alpha_{8}-\alpha_{9}$ gives dimension 6 and $\lambda-\alpha_{1}$ dimension 2. So $a_{i}=0$ for all $i \neq 9$.

Lemma 3.4. If $\lambda=\lambda_{n}$ (i.e. if we are in one of the remaining cases), then as an $X$-module, $W$ has high weight $2 \delta_{2}$ and $V$ is as in the statement of Theorem 3.1.

Proof. We still have to check cases 1) and 2) with $\lambda=\lambda_{n}$.

1) Assume $W$ has $T_{X}$-high weight $\delta_{1}+\delta_{3}$ and $p \neq 2$. Consider the embedding in a parabolic subgroup $P_{Y} \leq Y$ of the parabolic subgroup $P_{X} \leq X$ corresponding to $\left\{\beta_{1}, \beta_{2}\right\} \subseteq \Pi(X)$. Checking the dimensions at different $Q_{X}$-levels as before, we see that for any characteristic, $P_{Y}$ corresponds to $\left\{\alpha_{1}, \alpha_{2}, \alpha_{4}, \alpha_{5}, \alpha_{6}, \alpha_{7}\right\}$. Since $\lambda=\lambda_{n}, \operatorname{dim}\left(V /\left[V, Q_{Y}\right]\right)=16\left(V /\left[V, Q_{Y}\right]\right.$ is isomorphic to a spin module for the simple factor of $L_{Y}^{\prime}$ of type $B_{4}$ corresponding to $\left.\left\{\alpha_{4}, \alpha_{5}, \alpha_{6}, \alpha_{7}\right\}\right)$. The quotient $V /\left[V, Q_{Y}\right]$ is also an irreducible $L_{X}^{\prime}$-module (again by considering the action of $Z=Z\left(L_{X}\right)^{\circ}$ on the two $T_{X}$-high weight vectors of $V$ ), but $L_{X}^{\prime}=A_{2}$, which has no irreducible representations of dimension 16 in any characteristic. So we have no examples here.

2) Assume $\left.W\right|_{X}$ has high weight $2 \delta_{2}$. If $p=3$, then $\operatorname{dim}(W)=19$ and $Y=B_{9}$. Using $P_{X}$ corresponding to $\left\{\beta_{1}, \beta_{2}\right\}$ as above, we get an embedding of $P_{X}$ in the parabolic subgroup $P_{Y}$ corresponding to $\Pi(Y)-\left\{\alpha_{6}\right\}$. Since $\lambda=\lambda_{n}$, $\operatorname{dim}\left(V /\left[V, Q_{Y}\right]\right)=\operatorname{dim}\left(\operatorname{spin}\left(B_{3}\right)\right)=8$. But $V /\left[V, Q_{Y}\right]$ is an irreducible $L_{X}^{\prime}$-module (by the action of $Z$ again), and $L_{X}^{\prime}=A_{2}$, which has no 8-dimensional irreducible representations in characteristic 3 .

If $p \neq 3$, then take $P_{X}$ as above; again $P_{Y}$ corresponds to $\Pi(Y)-\left\{\alpha_{6}\right\}$ and $\operatorname{dim}\left(V /\left[V, Q_{Y}\right]\right)=\operatorname{dim}\left(\operatorname{spin}\left(D_{4}\right)\right)=8=\operatorname{dim}\left(V_{i} /\left[V_{i}, Q_{X}\right]\right)$ for $i=1$ or 2 , say $i=1$. So $b_{1}=1, b_{2}=1$.

Now let $P_{X}$ correspond to $\left\{\beta_{1}, \beta_{3}\right\}$. This $P_{X}$ is $t$-stable, so

$$
V /\left[V, Q_{Y}\right]=V /\left[V, Q_{X}\right]=V_{1} /\left[V_{1}, Q_{X}\right] \oplus V_{2} /\left[V_{2}, Q_{X}\right] .
$$

The embedding gives $P_{Y}$ corresponding to $\Pi(Y)-\left\{\alpha_{1}, \alpha_{5}\right\}$. So here,

$$
\begin{aligned}
\operatorname{dim}\left(V /\left[V, Q_{Y}\right]\right) & a=16=\operatorname{dim}\left(V /\left[V, Q_{X}\right]\right) \\
= & 2 \operatorname{dim}\left(V_{1} /\left[V_{1}, Q_{X}\right]\right) \\
= & 2\left(b_{1}+1\right)\left(b_{3}+1\right)=4\left(b_{3}+1\right) .
\end{aligned}
$$


So $b_{3}=3$. Now $\operatorname{dim}\left(V_{D_{10}}\left(\lambda_{n}\right)\right)=2^{9}$ in any characteristic; $\operatorname{dim}\left(V\left(3 \delta_{1}+\delta_{2}+\delta_{3}\right)\right)=$ $256=2^{8}$ when $p>7$ or $p=0$. So $\left.V\right|_{X}=\stackrel{3}{\bullet} \quad \begin{array}{lllll}\bullet & 1 & 1 & 1 & 3 \\ \bullet & \bullet\end{array},\left.V\right|_{Y}=\operatorname{spin}\left(D_{10}\right)$ is a possibility for $p \neq 2,3,5,7$.

In this case, consider again the embedding of $B_{X}$ in the parabolic subgroup

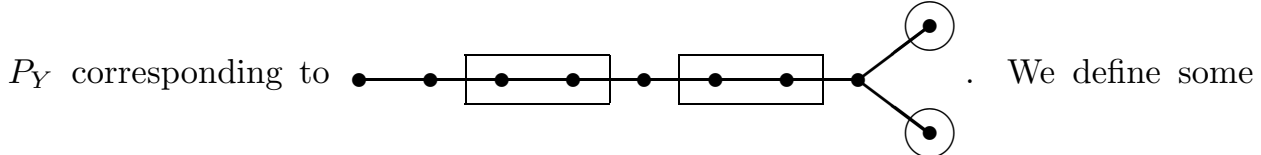
temporary notation: Let $\lambda_{1, j}=\left.\left(\lambda_{1}-\alpha_{1}-\cdots-\alpha_{j}\right)\right|_{T_{X}}$. By the construction of the embedding, we know that

$$
\begin{aligned}
\left.\lambda_{1}\right|_{T_{X}} & =\delta\left(=2 \delta_{2}\right), \\
\lambda_{1,1}=\left.\left(\lambda_{1}-\alpha_{1}\right)\right|_{T_{X}} & =\delta-\beta_{2}, \\
\left\{\lambda_{1,2}, \lambda_{1,3}, \lambda_{1,4}\right\} & =\left\{\delta-\beta_{1}-\beta_{2}, \delta-\beta_{2}-\beta_{3}, \delta-2 \beta_{2}\right\}, \\
\left\{\lambda_{1,5}, \lambda_{1,6}, \lambda_{1,7}\right\} & =\left\{\delta-\beta_{1}-2 \beta_{2}, \delta-2 \beta_{2}-\beta_{3}, \delta-\beta_{1}-\beta_{2}-\beta_{3}\right\}, \\
\left\{\lambda_{1,8}, \lambda_{1,9}, \lambda_{1,10}\right\} & =\left\{\delta-2 \beta_{2}-2 \beta_{3}, \delta-2 \beta_{1}-2 \beta_{2}, \delta-\beta_{1}-2 \beta_{2}-\beta_{3}\right\},
\end{aligned}
$$

with $\left.\left(\lambda_{1}-\alpha_{1}-\cdots-\alpha_{8}\right)\right|_{T_{X}}=-\left(\left.\left(\lambda_{1}-\alpha_{1}-\cdots-\alpha_{10}\right)\right|_{T_{X}}\right)\left(\right.$ since $\lambda_{1}-\alpha_{1}-\cdots-\alpha_{8}=$ $\left.-\lambda_{8}+\lambda_{9}+\lambda_{10}=-\left(\lambda_{1}-\alpha_{1}-\cdots-\alpha_{10}\right)\right)$.

This gives enough information to determine the possibilities for $\left.\alpha_{i}\right|_{T_{X}}$ for $i=$ $1, \ldots, 10$. We can write the $T_{Y}$-high weight for $V, \lambda_{10}$, in terms of the $\alpha_{i}$, and we find that with any of the possible choices made above, $\left\{\left.\lambda_{10}\right|_{T_{X}},\left(\lambda_{10}-\alpha_{10}\right)_{T_{X}}\right\}=$ $\left\{3 \delta_{1}+\delta_{2}+\delta_{3}, \delta_{1}+\delta_{2}+3 \delta_{3}\right\}$. So $V$ contains $A_{3}$-submodules of these two high weights; since their dimensions add to $\operatorname{dim}(V)$, we have the case stated in the theorem.

This completes the proof for $X=A_{3}$.

3.3. When Lemma 3.2 Doesn't Help. Using our standard construction of $P_{Y}$ (Lemma 2.7), the obvious situation in which the Lemma 3.2 is of no help is when $\delta=a \delta_{i}+b \delta_{j}$, i.e. when $U_{X}$-level 0 has dimension 2 . In this case $L_{1} \leq L_{Y}^{\prime}$ is of type $A_{1}$, corresponding to $\alpha_{2} \in \Pi(Y)$. Remember that $\delta$ must be symmetric, so that in fact the following is what we will need.

Lemma 3.5. The situation $\delta=a \delta_{i}+a \delta_{m-i+1}(i \leq m / 2), a_{2}=1$ does not give any examples in the Main Theorem if $m>3$.

Proof. With the given $\delta$ and with $P_{Y}$ as in Lemma 3.2, we have $\alpha_{2} \in \Pi\left(L_{Y}^{\prime}\right)$ since level 1 in the construction of $P_{Y}$ has dimension 2. So Lemma 3.2 tells us that $a_{2}$ is the only nonzero coefficient on $\Pi\left(L_{Y}^{\prime}\right)$.

Assume $i>1$. Consider the parabolic subgroup $P_{X}$ of $X$ corresponding to

$\Pi(X)-\left\{\beta_{1}, \beta_{m}\right\}$, as in the following picture: $\bullet \bullet \cdots \bullet$. So $L_{X}^{\prime}$ is of type $A_{m-2}$, and if we embed $P_{X} \hookrightarrow P_{Y}$ by the usual construction, then level 0 (that is, $\left.W /\left[W, Q_{X}\right]\right)$ of the flag which results is totally singular and has dimension $\geq 7$ (the smallest $W /\left[W, Q_{X}\right]$ could be is when $L_{X}^{\prime}$ is of type $A_{2}$ with a label of 1 on each node; this has dimension 7 in characteristic 3 and dimension 8 in other characteristics); thus $L_{1}$ (the simple factor of $L_{Y}^{\prime}$ corresponding to the quotient $W /\left[W, Q_{X}\right]$ in the flag) is of type $A_{l}$ for some $l \geq 6$. 
By Lemma 2.11, only one $L_{j}$ acts nontrivially on $V /\left[V, Q_{Y}\right]$. So $V /\left[V, Q_{Y}\right]$ is a nontrivial (there is at least one nonzero label on $L_{1}$ in the marking for the high weight of $V /\left[V, Q_{Y}\right]$, namely, on the second node), irreducible $L_{1}$-module, and as an $L_{X}^{\prime}$-module, $V /\left[V, Q_{Y}\right]$ is the sum of two irreducibles, $V_{1} /\left[V_{1}, Q_{X}\right]$ and $V_{2} /\left[V_{2}, Q_{X}\right]$. The natural module for $L_{1}$ is isomorphic to $W /\left[W, Q_{Y}\right]$ and is irreducible as an $L_{X^{-}}^{\prime}$ module. So unless $V_{1} /\left[V_{1}, Q_{X}\right] \cong V_{2} /\left[V_{2}, Q_{X}\right]$, we are in the situation we consider in this section and by induction no examples arise (the single case which arises below for $X=A_{3}$ does not arise inductively because $\delta$ does not have the form we are considering here).

Let the $T_{X}$-high weight of $V_{1}$ be $b_{1} \delta_{1}+b_{2} \delta_{2}+\cdots+b_{m} \delta_{m}$ (so the high weight of $V_{2}$ is $b_{m} \delta_{1}+b_{m-1} \delta_{2}+\ldots$ ). Assume $b_{2}=b_{m-1}, b_{3}=b_{m-2}, \ldots$ (i.e. $V_{1} /\left[V_{1}, Q_{X}\right] \cong$ $\left.V_{2} /\left[V_{2}, Q_{X}\right]\right)$. This implies $b_{1} \neq b_{m}$ since $V_{1} \neq V_{2}$. Now take $P_{X}$ to be another parabolic subgroup of $X$, corresponding to $\Pi(X)-\left\{\beta_{m}\right\}$, and embed $P_{X} \hookrightarrow P_{Y}$ via the same construction. Again we have $W /\left[W, Q_{Y}\right]=W /\left[W, Q_{X}\right]$ irreducible for $L_{X}^{\prime}$ and for $L_{1}$.

We show that in this case $V /\left[V, Q_{Y}\right]$ is irreducible as an $L_{X}$-module (in contrast to the situation when the parabolic subgroup of $X$ is $t$-stable, which forces $V /\left[V, Q_{Y}\right]$ to be the sum of two irreducibles for $\left.L_{X}\right)$. Let $Z=Z\left(L_{X}\right)^{\circ}$. Since $L_{Y}=C_{Y}(Z)$, we have $Z \leq Z\left(L_{Y}\right)$, so $Z$ induces scalars on $V /\left[V, Q_{Y}\right]$ (since $L_{Y}$ acts irreducibly on this module). But if $V /\left[V, Q_{Y}\right]$ is reducible as an $L_{X}$-module, then $V /\left[V, Q_{Y}\right]=$ $V_{1} /\left[V_{1}, Q_{X}\right] \oplus V_{2} /\left[V_{2}, Q_{X}\right]$, and we show that $Z$ acts differently on these two $L_{X^{-}}$ modules:

$$
\begin{aligned}
Z & =\left\{\operatorname{diag}\left(a, \ldots, a, a^{-m}\right) \mid a \in K^{*}\right\} \\
& =\left\{h_{\beta_{1}}(a) h_{\beta_{2}}\left(a^{2}\right) \ldots h_{\beta_{m}}\left(a^{m}\right) \mid a \in K^{*}\right\} .
\end{aligned}
$$

The two $X$-modules $V_{1}, V_{2}$ have high weight labelling

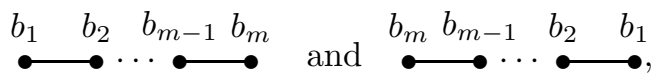

so $h_{\beta_{1}}(a) h_{\beta_{2}}\left(a^{2}\right) \ldots h_{\beta_{m}}\left(a^{m}\right)$ acts as multiplication by $a^{b_{1}+2 b_{2}+\cdots+m b_{m}}$ on a high weight vector $v_{1} \in V_{1}$ and as multiplication by $a^{b_{m}+2 b_{m-1}+\cdots+m b_{1}}$ on a high weight vector $v_{2} \in V_{2}$; these two exponents are not equal. Since $v_{j}$ has a nonzero image in $V_{j} /\left[V_{j}, Q_{X}\right]$, this shows that only one of the $V_{j} /\left[V_{j}, Q_{Y}\right]$ can have a nonzero image in $V /\left[V, Q_{Y}\right]$. So $V /\left[V, Q_{Y}\right]$ is irreducible as an $L_{X}$-module.

Assume $V_{1}$ is the summand which projects nontrivially to $V /\left[V, Q_{Y}\right]$ (so $V_{2} \subseteq$ $\left.\left[V, Q_{Y}\right]\right)$. As no irreducible restricted representations of $A_{m-1}$ are tensor decomposable by Lemma 2.1, $L_{1}$ is the only simple factor of $L_{Y}^{\prime}$ to act nontrivially on $V /\left[V, Q_{Y}\right]$. Note that the rank of $L_{1}$ is $\geq 15$, since this rank is one less than the dimension of $W /\left[W, Q_{Y}\right]$ : With $\delta$ of the form we are assuming, the $A_{m-1}$-high weight of $W /\left[W, Q_{Y}\right]$ is of the form $\left.\left(a \delta_{i}+a \delta_{m-i+1}\right)\right|_{T_{L_{X}^{\prime}}}$. The Weyl module for $A_{m-1}$ with this high weight has at least 16 weights, and these weights all appear in the irreducible module $W /\left[W, Q_{Y}\right]$ by the result in [12].

So now we are inductively in the situation examined in $[9]: V /\left[V, Q_{Y}\right]$ is an irreducible module for both $L_{X}^{\prime}$ and $L_{1}$, and it is not the natural module for $L_{1}$ (since in the labelling for the $L_{1}$-high weight of $V /\left[V, Q_{Y}\right]$ there is a 1 on the second node of $\left.\Pi\left(L_{1}\right)\right)$. Also, $L_{X}^{\prime} \neq L_{1}$ because the natural module for $L_{1}, W /\left[W, Q_{Y}\right]$, has high weight $\left.\delta\right|_{T_{L_{X}^{\prime}}}$ and thus has dimension larger than $m$, which is the dimension of the natural module for $L_{X}^{\prime}$. So any examples here would appear in Table 1 of [9]; 
examining that table, we see that in fact there are no examples. This completes the case $i>1$.

So we need to consider only the case $i=1$, i.e. $\delta=a \delta_{1}+a \delta_{m}$, with $\lambda=\lambda_{2}+\cdots$. Let $P_{X}=L_{X} Q_{X}$ be the maximal parabolic subgroup of $X$ corresponding to $\beta_{j} \in$ $\Pi(X)$ and embed $P_{X}$ in a parabolic subgroup $P_{Y}=L_{Y} Q_{Y}$ of $Y$ via the usual construction. Notice that $L_{1}$ (the simple factor of $L_{Y}^{\prime}$ corresponding to the $Q_{X^{-}}$ level 0 of $W$ ) is of type $A_{l}$, with $l>3$ unless $m=4$ (we have taken care of the cases $m=2,3$ in $\S 3.2)$. We wish to show that for at least one choice of $j, V /\left[V, Q_{Y}\right]$ is irreducible as an $L_{X}$-module. We will again use the action of $Z=Z\left(L_{X}\right)^{\circ}$ on $T_{X}$-high weight vectors in $V$ :

$$
\begin{aligned}
Z= & \left\{\operatorname{diag}(\underbrace{a^{(m-j+1)}, a^{(m-j+1)}, \ldots, a^{(m-j+1)}}_{j}, \underbrace{a^{-j}, a^{-j}, \ldots, a^{-j}}_{m-j+1}) \mid a \in K^{*}\right\} \\
= & \left\{h_{\beta_{1}}\left(a^{(m-j+1)}\right) h_{\beta_{2}}\left(a^{2(m-j+1)}\right)\right. \\
& \left.\ldots h_{\beta_{j}}\left(a^{j(m-j+1)}\right) h_{\beta_{j+1}}\left(a^{j(m-j)}\right) h_{\beta_{j+2}}\left(a^{j(m-j-1)}\right) \ldots h_{\beta_{m}}\left(a^{j}\right) \mid a \in K^{*}\right\} .
\end{aligned}
$$

If $V /\left[V, Q_{Y}\right]$ is reducible as an $L_{X}$-module, then as above, $Z$ must act as multiplication by the same scalar on a high weight vector $v_{1} \in V_{1}$ as on a high weight vector $v_{2} \in V_{2}$, and we get the equation

$$
\begin{aligned}
&(m-j+1) b_{1}+2(m-j+1) b_{2}+\cdots+j(m-j+1) b_{j} \\
&+ j(m-j) b_{j+1}+j(m-j-1) b_{j+2}+\cdots+j \cdot 1 \cdot b_{m} \\
&=(m-j+1) b_{m}+2(m-j+1) b_{m-1}+\cdots+j(m-j+1) b_{m-j+1} \\
& \quad+j(m-j) b_{m-j}+j(m-j-1) b_{m-j-1}+\cdots+j \cdot 1 \cdot b_{1} .
\end{aligned}
$$

If we assume that $V /\left[V, Q_{Y}\right]$ is reducible as an $L_{X}$-module for every $j$, then we have a system of equations which together imply $b_{1}=b_{m}, b_{2}=b_{m-1}, \ldots$ For example, the equations for $j=m$ and $j=m-1$ are

$$
\begin{aligned}
b_{1}+2 b_{2}+\cdots+(m-1) b_{m-1}+m b_{m} & =b_{m}+2 b_{m-1}+\cdots+m b_{1}, \\
2 b_{1}+4 b_{2}+\cdots+2(m-1) b_{m-1}+(m-1) b_{m} & =2 b_{m}+4 b_{m-1}+\cdots+(m-1) b_{1} .
\end{aligned}
$$

Twice the first equation minus the second gives $(m+1) b_{m}=(m+1) b_{1}$. Knowing $b_{1}=b_{m}$, the equations for $j=m$ and $j=m-2$ give $b_{2}=b_{m-1}$; continuing in this manner we obtain $b_{l}=b_{m-l+1}$ for every $l$. But this is impossible, as it would imply that $V$ is reducible for $X\langle t\rangle$. So for some $j, 1 \leq j \leq m, V /\left[V, Q_{Y}\right]$ is irreducible as an $L_{X}^{\prime}$-module, where $L_{X}, Q_{Y}$ are as above.

But then again we are in the situation examined in $[9]: V /\left[V, Q_{Y}\right]$ is an irreducible module for $L_{X}^{\prime}=A_{j-1} \times A_{m-j}$ and for $L_{1}$. So one of the following occurs:

1. The embedding $L_{X} \hookrightarrow L_{1}$ is an isomorphism.

2. $V /\left[V, Q_{Y}\right]$ is the natural module for $L_{1}$ (which happens only for $m=3,4$, since $L_{1}$ has rank $\geq 4$ in other cases we consider and the $L_{1}$-high weight of $V /\left[V, Q_{Y}\right]$ has a nonzero label on the node $\left.\alpha_{2}\right)$.

3. $V /\left[V, Q_{Y}\right]$ appears in Table 1 of $[9]$.

We deal with 3 first. Of the appearances of the inclusion $A_{j-1} \times A_{m-j} \leq A_{l}$ in Table 1 of [9], only one (case $I_{7}$ there) gives the correct restriction of the natural module for $A_{l}$ to the subgroup. So the possible picture here is $\left(L_{X}^{\prime}=A_{m-1}\right.$; 
$L_{1}=A_{\left(m^{2}+m-2\right) / 2}$; the high weight of $\left.\left.W\right|_{X}=2 \delta_{1}+2 \delta_{m}\right)$ :

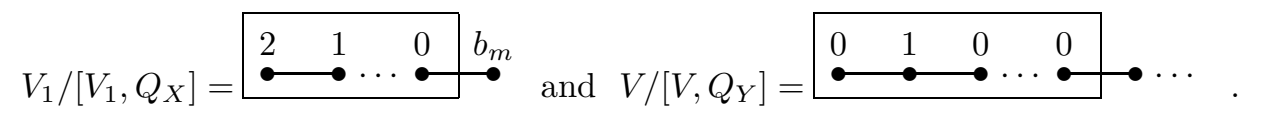

Now we look at yet another parabolic subgroup of $X$ : Let $P_{X}$ be generated by $B_{X}$ and the root subgroups for $\beta_{1}$ and $\beta_{m}$; embed $P_{X}$ in a parabolic subgroup $P_{Y}$ of $Y$ as usual. By Lemma 2.11, $L_{1}$ is the only simple factor of $L_{Y}^{\prime}$ to act nontrivially on $V /\left[V, Q_{Y}\right]$. Notice $\operatorname{dim}\left(W /\left[W, Q_{X}\right]\right)=9\left(\right.$ since $\left.W\right|_{X}$ has $T_{X}$-high weight $\left.2 \delta_{1}+2 \delta_{m}\right)$, so $L_{1}$ has rank 8 . Thus $2 \cdot 3\left(b_{m}+1\right)=\operatorname{dim}\left(V_{1} /\left[V_{1}, Q_{X}\right]\right)+\operatorname{dim}\left(V_{2} /\left[V_{2}, Q_{X}\right]\right)=$ $\operatorname{dim}\left(V /\left[V, Q_{X}\right]\right)=\operatorname{dim}\left(V /\left[V, Q_{Y}\right]\right)=\left(\begin{array}{l}9 \\ 2\end{array}\right)=36$, which tells us $b_{m}=5$. This tells us that the only two $T_{L_{X}^{\prime}}$-high weights of $V /\left[V, Q_{Y}\right]$ are $\left(2 \delta_{1}, 5 \delta_{m}\right)$ and $\left(5 \delta_{1}, 2 \delta_{m}\right)$. But in fact $V /\left[V, Q_{Y}\right] \cong \bigwedge^{2}\left(W /\left[W, Q_{Y}\right]\right)(p \neq 2$ because of the 2 appearing in the picture above and our assumption that the $T_{X}$-high weights of $V$ are restricted), and two $T_{L_{X}^{\prime}}$-high weights of $\bigwedge^{2}\left(W /\left[W, Q_{Y}\right]\right)$ are $\left(4 \delta_{1}, 2 \delta_{m}\right)$ and $\left(2 \delta_{1}, 4 \delta_{m}\right)$. Again we have a contradiction.

Next consider item 2. This can only occur if $\operatorname{dim}\left(W /\left[W, Q_{X}\right]\right) \leq 4$ (since there is a 1 on the second node in the marking for the high weight of $V /\left[V, Q_{Y}\right]$ on $L_{1}$ ) and this occurs only in the cases we excluded $\left(X=A_{2}, A_{3}\right)$ and the case $X=A_{4}$, with $j=4$ (or, equivalently, $j=1$ ), $\delta=\delta_{1}+\delta_{4}$. But this in fact gives an instance of item 1 (it implies that $W /\left[W, Q_{Y}\right]$ has $T_{L_{X}^{\prime}}$-high weight $\delta_{1}$ ).

So we are left with item 1 . Note that the equations (1) hold for all $j \neq 1, m$, since in the consideration of item 3 above we obtained a contradiction to $V /\left[V, Q_{Y}\right]$ being irreducible for $L_{X}^{\prime}$ when $j \neq 1, m$. We have the pictures

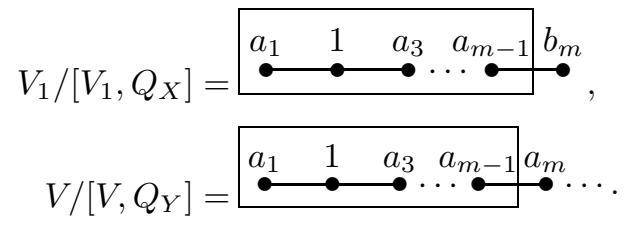

So $a_{1}=b_{1}, 1=a_{2}=b_{2}, \ldots, a_{m-1}=b_{m-1}$. Now let $P_{X}$ correspond to the indicated nodes, $\bullet \longrightarrow \cdots \bullet$, and embed it in a parabolic subgroup of $Y$ as usual. Then $V /\left[V, Q_{Y}\right]$ is the sum of two irreducible $L_{X}^{\prime}$-modules, and the simple factor $L_{2}$ of $L_{Y}^{\prime}$ corresponds to the indicated nodes at the beginning of the Dynkin

diagram for $Y, \stackrel{L_{2}}{\bullet \bullet \cdots \bullet \bullet} \stackrel{L}{3}^{2} \bullet \cdots, \cdots$, with $L_{2}$ of rank $2 m-3$. But by the construction of $P_{Y}$, we know the embedding $L_{X}^{\prime} \hookrightarrow L_{2}$ here (the natural module for $L_{2}$ is $Q_{X}$-level 1 of $W$ and restricts to $L_{X}^{\prime}$ as the sum of the two irreducible modules with high weights $\left.\delta_{2}\right|_{T_{L_{X}^{\prime}}}$ and $\left.\left.\delta_{m-1}\right|_{T_{L_{X}^{\prime}}}\right)$. As this situation gave no examples in [4], we know by induction that either 1) $a_{3}=a_{4}=\cdots=a_{2 m-2}=0$ (i.e. $V /\left[V, Q_{Y}\right] \cong$ the natural module for $L_{2}$ ) or 2) $1=a_{2}=a_{m-1}, a_{3}=a_{m-2}, \ldots$ (i.e. $V /\left[V, Q_{Y}\right]$ is reducible for $\left.L_{X}^{\prime}\langle t\rangle\right)$.

We noted that all the equations (1) on the previous page hold for $j \neq 1, m$. The equation for $j=m-1$ is

$$
2 b_{1}+4 b_{2}+\cdots+2(m-1) b_{m-1}+(m-1) b_{m}=2 b_{m}+4 b_{m-1}+\cdots+(m-1) b_{1} .
$$


Now if 2) above holds, we know that $b_{2}=b_{m-1}, b_{3}=b_{m-2}, \ldots$ (remember that $a_{i}=b_{i}$ for $1<i<m$ ), which together with the above equation gives $b_{1}=b_{m}$, which is a contradiction. So 2) does not occur.

If $a_{3}=\cdots=a_{2 m-2}=0$, i.e. 1$)$ holds, then once again we examine the equation above and see that now it reduces to $2 b_{1}+4+(m-1) b_{m}=2 b_{m}+2(m-1)+(m-1) b_{1}$ (remembering $a_{i}=b_{i}$ for $1<i<m$ and $a_{2}=b_{2}=1$ ) or $b_{m}-b_{1}=2$. Let $P_{X}$ be the parabolic subgroup of $X$ corresponding to $\left\{\beta_{1}, \beta_{m}\right\}$. Then $L_{1}$ is of type $A_{3}, L_{1}$ is the only factor of $L_{Y}^{\prime}$ acting nontrivially on $V /\left[V, Q_{Y}\right]$ by Lemma 2.11, and the picture is

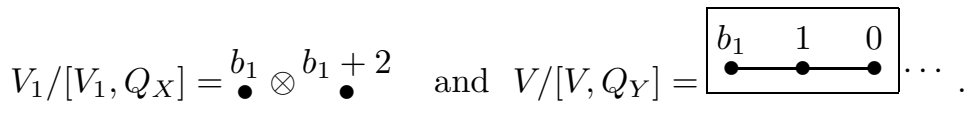

By the Andersen-Jantzen sum formula ([1]), the $A_{3}$-module with the high weight pictured above is in fact the Weyl module except when $b_{1}=p-2$. But $p>$ $b_{m}=b_{1}+2$ here, so the dimension of $V /\left[V, Q_{Y}\right]$ is the dimension of the Weyl module, which is $\left(b_{1}+1\right)\left(b_{1}+3\right)\left(b_{1}+4\right) / 2$. On the other hand, $\operatorname{dim}\left(V /\left[V, Q_{Y}\right]\right)=$ $\operatorname{dim}\left(V /\left[V, Q_{X}\right]\right)=2 \operatorname{dim}\left(V_{1} /\left[V_{1}, Q_{X}\right]\right)=2\left(b_{1}+1\right)\left(b_{1}+3\right)$. These two equations together imply $b_{1}=0$.

So now all the $b_{i}$ are known: $b_{2}=1, b_{m}=2$, and all others are 0 . Since one of the coefficients is greater than 1 , we know $p \neq 2$. And since the $T_{X}$-high weight of $W$ is $\delta_{1}+\delta_{m}$, we know $\operatorname{dim}(W)=m(m+2)$ or $m(m+2)-1$. We claim that $\operatorname{dim}(V) \geq \operatorname{dim}\left(\bigwedge^{2} W\right)$ (this is clear if $\lambda=\lambda_{2}$, as then $V=\bigwedge^{2} W$ since $Y=D_{n}$ and $p \neq 2$ ).

The $T_{Y}$-high weight $\lambda$ of $V$ has a $\lambda_{2}$-coefficient of 1 , and $W$ is orthogonal. We claim that any $B_{n}$ or $D_{n}$ weight of this form has one of $\lambda_{2}, \lambda_{3}$, or $\lambda_{n}$ as a subdominant weight. For $B_{n}$, every fundamental weight except $\lambda_{n}$ is a sum of roots, and $2 \lambda_{n}$ is a sum of roots, so any $\lambda$ with a $\lambda_{2}$-coefficient of 1 has $\lambda_{2}$ or $\lambda_{n}$ as a subdominant weight. For $D_{n}$, any fundamental weight $\lambda_{k}$ for even $k \leq n-2$ is a sum of roots; for odd $k$ with $1<k \leq n-2, \lambda_{k}$ differs from a sum of roots by $\lambda_{3}$. The weight $2 \lambda_{n}$ or $2 \lambda_{n-1}$ is either a sum of roots or differs from one by $\lambda_{3}$; finally, $\lambda_{1}+\lambda_{2}$ has $\lambda_{3}$ as a subdominant weight. So the claim holds.

Say $\lambda \succ \lambda_{i}$. Then by the result in [8], every weight which appears in the Weyl module with $T_{Y}$-high weight $\lambda$ appears in $V$. So

$$
\begin{aligned}
& \operatorname{dim}(V) \geq \operatorname{card}\left(\left\{\omega \text { is a weight occurring in } V_{Y}\left(\lambda_{i}\right)\right\}\right) \\
& \quad+\operatorname{card}(\text { Weyl group-orbit of } \lambda) \\
& \geq\left(\left(\begin{array}{c}
\operatorname{dim}(W) \\
2
\end{array}\right)-\operatorname{dim}(W)\right)+\operatorname{dim}(W)=\operatorname{dim}\left(\bigwedge^{2} W\right) .
\end{aligned}
$$

Now we have a chain of inequalities (the second line is a computation of the dimension of the Weyl module with the specified high weight, using the Weyl dimension formula):

$$
\begin{aligned}
& \operatorname{dim}(V)=2 \operatorname{dim}\left(V_{A_{m}}\left(\lambda_{2}+2 \lambda_{m}\right)\right) \\
& \leq 2((m-1) m(m+2)(m+3) / 4) \\
& =\left(m^{4}+4 m^{3}+m^{2}-6 m\right) / 2 \\
& <\left(m^{4}+4 m^{3}+m^{2}-6 m+2\right) / 2 \\
& =\left(\begin{array}{c}
m(m+2)-1 \\
2
\end{array}\right) \\
& \leq \quad(\underset{2}{\operatorname{dim}(W)}) \\
& \leq \operatorname{dim}^{2}(V),
\end{aligned}
$$


which is a contradiction. So we have ruled out all possible configurations, and the proof of the lemma is complete.

3.4. The General $X=A_{m}$ Case. We must prove that there are no triples $(X, Y, V)$ with $X$ acting irreducibly on $W$ and $t$ acting on $W$, for $X$ of type $A_{m}$ with $m>3$. We use the same argument as in the $A_{2}$ and $A_{3}$ cases to limit the possibilities for the embeddings $X \hookrightarrow Y$, relying on Lemma 3.2. Lemma 3.5 tells us we need not worry about level 1 in the computation of dimensions of $U_{X}$-levels.

As in the small cases, the method we use to generate weights that appear in a representation is simple: If $\mu$ is a weight in the $X$-module $M$ and $\left\langle\mu, \beta_{i}\right\rangle \geq a>0$, then $\mu-a \beta_{i}$ is another weight appearing in $M$.

Every symmetric weight for $X=A_{m}$ except $\delta_{1}+\delta_{m}$ has either $\delta_{2}+\delta_{m-1}$ or $\delta_{(m+1) / 2}$ as a subdominant weight $\left(\delta_{i}+\delta_{m-i+1}\right.$ is a sum of roots for any $\left.1 \leq i \leq m\right)$. It is relatively simple to find enough weights in each level for $\delta=\delta_{2}+\delta_{m-1}$ : The level $l_{\delta}$ of the low weight $-\delta$ in this case is $2(m+(m-2))=4(m-1)$, so we must show there are at least three weights at level $j$ for $2 \leq j<2(m-1)$ and at least 5 at level $2(m-1)$; this is easy. Unless otherwise stated, the levels we discuss are $U_{X}$-levels.

The case $\delta=\delta_{(m+1) / 2}(m$ odd $)$ is considerably more difficult. At $U_{X}$-level 2 there are only the two weights $\delta-\beta_{(m+1) / 2}-\beta_{(m-1) / 2}$ and $\delta-\beta_{(m+1) / 2}-\beta_{(m+3) / 2}$; so Lemma 3.2 is of no help here. We have $l_{\delta}=(m+1)^{2} / 4$, so if we can show that there are at least 3 weights at each level $3 \leq j<(m+1)^{2} / 8$ and at least 5 at level $(m+1)^{2} / 8$ (when this is integral), we will only have level 2 to worry about.

Notice that for any weight $\delta$, the dimensions and numbers of weights of $U_{X^{-}}$ levels of the $X$-module with high weight $\delta$ are symmetric about level $l_{\delta} / 2$. In other words, $\operatorname{dim}\left(W_{i}\right)=\operatorname{dim}\left(W_{l_{\delta}-i}\right)$ and the same numbers of weights appear in these two spaces, since $w_{0}$ interchanges them. So, for instance, if $V_{A_{l}}(\delta)$ has at least 3 weights at all levels $j$ for $i \leq j \leq l_{\delta} / 2$, then it has at least 3 at all levels $j$ for $i \leq j \leq l_{\delta}-i$.

For $m=5,7$, it is easy to see that there are enough weights at levels 3 through $l_{\delta} / 2$ (three at every level except level 8 for $m=7$, in which case there are at least 5 weights). We proceed by induction on $m$ (considering the subsystem group of $X$ of type $A_{m-2}$, corresponding to $\left.\Pi(X)-\left\{\beta_{1}, \beta_{m}\right\}\right)$. Assume $\delta=\delta_{(m+1) / 2}$ for $m \geq 9$. Notice that $\delta_{1}-\delta_{(m+1) / 2}+\delta_{m}$ is a weight at level $l_{\left(\left.\delta_{(m+1) / 2}\right|_{A_{m-2}}\right)}=(m-1)^{2} / 4$ and that if $\delta_{(m+1) / 2}-\sum_{i=2}^{m-1} c_{i} \beta_{i}$ is a weight at level $j$ in the $A_{m-2}$-module with high weight $\delta_{(m+1) / 2}$, then $\delta_{(m+1) / 2}-\sum_{i=2}^{m-1} c_{i} \beta_{i}$ is a weight at level $j$ in $W$ for $\delta=\delta_{(m+1) / 2}$. So, using the comment in the last paragraph and by induction from the $A_{n-2}$ case, $W$ has at least three weights at levels 3 through $\left((m-1)^{2} / 4\right)-2$. But $\left((m-1)^{2} / 4\right)-2>(m+1)^{2} / 8$ for $m>7$, which shows that the only possibilities for a simple factor of $L_{Y}^{\prime}$ of type $A_{1}$ are the above-mentioned factor corresponding to $U_{X}$-level 2 and level $(m+1)^{2} / 8$ when this number is integral.

We need to show that there are at least 5 weights at level $(m+1)^{2} / 8$ when $4 \mid m+1$ and $\delta=\delta_{(m+1) / 2}$. It is easy to write down 5 such weights for $m=7$, as noted above; in particular in this case there are two at this level which are symmetric (with respect to the graph automorphism). So assume $m \geq 11$ and consider the $A_{m-4^{-}}$ subsystem subgroup of $X$, corresponding to $\Pi(X)-\left\{\beta_{1}, \beta_{2}, \beta_{m-1}, \beta_{m}\right\}$. Assume 
there are at least two symmetric weights at level $(m-3)^{2} / 8$ for the $A_{m-4}$-module with high weight $\delta_{(m+1) / 2}$; as in the last paragraph each corresponds to a weight of $W$ at level $(m-3)^{2} / 8$. Let $\gamma$ be one of these weights of $W$. Write $\gamma=\delta-\sum_{i=3}^{m-2} c_{i} \beta_{i}$. For any symmetric weight of $W$ expressed in this form, either

1) $c_{(m+1) / 2}=c_{(m-1) / 2}+1\left(=c_{(m+3) / 2}+1\right)$ or

2) $c_{(m+1) / 2}=c_{(m-1) / 2}\left(=c_{(m+3) / 2}\right)$.

If $\gamma$ satisfies 1), then $\gamma-\beta_{2}-\beta_{3}-\cdots-\beta_{(m-1) / 2}-2 \beta_{(m+1) / 2}-\beta_{(m+3) / 2}-\cdots-\beta_{m-1}$ is a symmetric weight of $W$ at level $(m-3)^{2} / 8+m-1=(m+1)^{2} / 8$ which satisfies $2)$; if $\gamma$ satisfies 2), then $\gamma-\beta_{1}-\beta_{2}-\cdots-\beta_{(m-1) / 2}-\beta_{(m+3) / 2}-\cdots-\beta_{m}$ is such a weight which satisfies 1$)$. Since of the two symmetric weights at level 8 for $m=7$, one $\left(\delta_{4}-\beta_{1}-\beta_{2}-\beta_{3}-2 \beta_{4}-\beta_{5}-\beta_{6}-\beta_{7}\right)$ satisfies 1$)$ and the other $\left(\delta_{4}-\beta_{2}-2 \beta_{3}-2 \beta_{4}-2 \beta_{5}-\beta_{6}\right)$ satisfies 2$)$, we conclude that the two symmetric weights at level $(m+1)^{2} / 8$ we obtain are in fact distinct. At the same level we have the weights $\gamma-\beta_{1}-\cdots-\beta_{m-1}$ and $\gamma-\beta_{2}-\cdots-\beta_{m}$. So from each of the two symmetric weights at level $(m-3)^{2} / 8$, we obtain 3 weights at level $(m+1)^{2} / 8$; the six weights occurring are all distinct. So there are no possible $A_{1}$ factors of $L_{Y}^{\prime}$ here.

Note that $\delta=\delta_{(m+1) / 2}$ is the only possible high weight of $W$ which does not have at least 3 weights at level 2. Now the induction is much the same as for $X=A_{3}$. Recall that $\delta=\sum d_{i} \delta_{i}$. If $d_{(m+1) / 2}>1$, then $\delta-\beta_{(m+1) / 2}$ is lower in the partial order and still has one of $\delta_{2}+\delta_{m-1}, \delta_{(m+1) / 2}$ as a subdominant weight, so by induction $\delta$ has enough weights at levels 4 through $\left(l_{\delta} / 2\right)-1$, and we can easily check levels 1,2 , and 3 .

If $d_{(m+1) / 2} \leq 1$ or $m$ is even, then let $k$ be such that $d_{k} \neq 0$ but $d_{i}=0$ for all $k<i<(m+1) / 2$. Then $\delta-\beta_{k}-\beta_{k+1}-\cdots-\beta_{m-k+1}($ at level $m-2 k+2)$ is lower in the partial order and still has one of $\delta_{2}+\delta_{m-1}, \delta_{(m+1) / 2}$ as a subdominant weight, so by induction $\delta$ has enough weights at levels $m-2 k+4$ and higher. Once again, the missing levels are easy to check.

So the cases not ruled out by the induction are $\delta=\delta_{1}+\delta_{m}$ and $\delta=\delta_{(m+1) / 2}$ at level 2. For the first case, we can easily write down enough weights in levels 2 through $m-1=\left(l_{\delta} / 2\right)-1$. At level $m$ the only weight is 0 , but the dimension of its weight space is $m$ or (if $p \mid m+1) m-1$. So the only possibilities for a $U_{X}$-level of dimension 4 or less are $m=4$, or $m=5$ with $p=2$ or 3 .

Consider the case $\delta=\delta_{(m+1) / 2}(m \geq 5)$. In the labelling for the $Y$-high weight of $V$, there is a 1 on the third node of the Dynkin diagram. Let $P_{X}$ correspond to $\Pi(X)-\left\{\beta_{1}, \beta_{m}\right\}$. Then $\operatorname{dim}\left(W /\left[W, Q_{X}\right]\right) \geq 6$, so the corresponding simple factor $L_{1}$ of $L_{Y}^{\prime}$ is of type $A_{l}$ for some $l \geq 5$, and $V /\left[V, Q_{Y}\right]$ is an irreducible $L_{1}$-module whose high weight has a $\lambda_{3}$-coefficient of $1\left(L_{1}\right.$ is the only simple factor of $L_{Y}^{\prime}$ to act on $V /\left[V, Q_{Y}\right]$ by Lemma 2.11). The natural module for $L_{1}$ is isomorphic to $W /\left[W, Q_{Y}\right]$ and is irreducible as an $L_{X}^{\prime}$-module, while $V /\left[V, Q_{Y}\right]$ is the sum of two irreducible modules for $L_{X}^{\prime}$, interchanged by $t$. Since $V /\left[V, Q_{Y}\right] \nRightarrow$ the natural module for $L_{1}$ and no cases with this configuration appeared for $X=A_{3}$, by induction we know that $V /\left[V, Q_{Y}\right]$ must not be irreducible for $L_{X}^{\prime}\langle t\rangle$. So $b_{2}=b_{m-1}, b_{3}=b_{m-2}$, etc. $\left(\left.V_{1}\right|_{X}=V_{A_{m}}\left(b_{1} \delta_{1}+\cdots+b_{m} \delta_{m}\right)\right)$.

Now let $P_{X}$ correspond to $\Pi(X)-\left\{\beta_{m}\right\}$. By the same construction (using the action of $Z$ on high weight vectors) $V /\left[V, Q_{Y}\right]$ is irreducible as an $L_{X}^{\prime}$-module. The rank of $L_{1}$ is at least 9 (since $W /\left[W, Q_{Y}\right] \cong$ the $A_{m-1}$-module with high weight $\left.\lambda_{(m+1) / 2}\right)$, and $V /\left[V, Q_{Y}\right]$ is irreducible as an $L_{1}$-module, with a 1 on the third 
node of the Dynkin diagram in its high weight labelling $\left(L_{1}\right.$ is the only factor of $L_{Y}^{\prime}$ acting nontrivially on $V /\left[V, Q_{Y}\right]$ since no restricted irreducible $A_{m-1}$-modules are tensor decomposable). So we are again in the case examined in [9]. Examining Table 1 there, we see that there are no examples of the configuration we obtain. So $\delta=\delta_{(m+1) / 2}$ does not occur.

The two cases that remain are 1) $m=4, \delta=\delta_{1}+\delta_{4}$, with the $T_{Y}$-high weight $\lambda$ of $V$ having a $\lambda_{n}$-coefficient of 1 ( $Y$ has type $D_{12}(p \neq 2,5), B_{11}(p=5)$, or possibly $C_{12}(p=2)$ ); and 2) $m=5, p=5, \delta=\delta_{1}+\delta_{5}$, $\lambda$ with a $\lambda_{17^{-}}$ coefficient of 1 ( $Y$ has type $D_{17}$ ). In both these cases, we can use the fact that $\operatorname{dim}\left(V^{2}\left(Q_{Y}\right)\right) \leq m \operatorname{dim}\left(V^{1}\left(Q_{Y}\right)\right)$ (Lemma 2.9) to conclude that in fact $\lambda=\lambda_{n}$; i.e. $V$ is a spin module for $Y$.

For 1), we first let $P_{X}$ correspond to $\left\{\beta_{2}, \beta_{3}\right\} \subseteq \Pi(X)$ and conclude that $b_{2}=b_{3}$ as the resulting configuration does not appear in [4]. Next let $P_{X}$ correspond to $\Pi(X)-\left\{\beta_{4}\right\}$. Then again the construction using the action of $Z$ tells us that $V /\left[V, Q_{Y}\right]$ is irreducible as an $L_{X}^{\prime}$-module, and we are back in the situation examined in [9]. Examining Table 1 of [9], we see that there are no examples of modules irreducible for both $A_{3} \leq D_{8}$.

For 2), we use the same constructions and conclude that to have an example, there must be an example of the form $A_{4} \leq D_{12}$ in Table 1 of [9]; there is none. So this final possibility is ruled out.

This completes the proof of the theorem.

\section{The CASE $X=D_{m}$}

In this section we establish the main result for the case $X=D_{m}, G=X\langle t\rangle$. Section 3 included the base case of $X=D_{3}=A_{3}$. So throughout this section $X=D_{m}$ for $m>3$ and $G=X\langle t\rangle$. We assume that $t$ acts on $W$, the natural module for $Y$.

All notation ( $X \leq Y, V, V_{1}, V_{2}, \lambda, \delta$, etc.) is as in previous sections. Recall that $\Pi(X)=\left\{\beta_{i}\right\}$ is the set of simple roots of $X ; \Pi(Y)=\left\{\alpha_{i}\right\}$ is the set of simple roots of $Y$, and $n$ is the rank of $Y$. The main theorem of this section is

Theorem 4.1. If $X$ acts irreducibly on the natural module $W$ for $Y$ and $X$ is of type $D_{m}$ for $m>3$, then $p=2, Y=C_{m}$ or $B_{m}, W$ is the natural module for $X$ and $Y$; and $V$ is the spin module for $Y$, the sum of two spin modules for $X$. This is $U_{7}$ in Table 2.

Proof. Assume $X$ is of type $D_{m}$. Let $\delta$ be the $X$-high weight of $W$. Since we are assuming $t$ acts on $W$, we have that $\delta$ is symmetric with respect to $t$, so by $[9,1.8]$, $X$ stabilizes a non-degenerate bilinear form on $W$. So $Y$ is of type $B_{n}, C_{n}$, or $D_{n}$.

Let $P_{X}$ be the maximal parabolic subgroup of $X$ corresponding to $\beta_{1}$. Then $L_{X}^{\prime}$ is of type $D_{n-1}$, and we embed $P_{X}$ in $P_{Y}$, a parabolic subgroup of $Y$, via the construction detailed in Lemma 2.7. Then $Q_{X} \subseteq Q_{Y}$ and $L_{X}^{\prime} \subseteq L_{Y}^{\prime}$ for $L_{Y}=C_{Y}\left(Z\left(L_{X}\right)^{\circ}\right)$. By Lemma 2.8, $L_{Y}$ is $t$-stable. Write $L_{Y}^{\prime}=L_{1} \times \cdots \times L_{m}$ where each $L_{i}$ is simple. By Lemma 2.2, $V /\left[V, Q_{Y}\right]$ is irreducible as an $L_{Y}^{\prime}$-module with high weight $\left.\lambda\right|_{T_{Y} \cap L_{Y}^{\prime}}$; also, Lemma 2.11 tells us that only one $L_{i}$ acts nontrivially on $V /\left[V, Q_{Y}\right]$. Note that since $P_{X}$ is $t$-stable, Lemma 2.8 tells us that $V /\left[V, Q_{Y}\right]=V /\left[V, Q_{X}\right]=V_{1} /\left[V_{1}, Q_{X}\right] \oplus V_{2} /\left[V_{2}, Q_{X}\right]$ is a sum of two irreducible $L_{X}^{\prime}$-modules; neither of these $L_{X}^{\prime}$ summands is trivial, since that would imply that $V_{1} \cong V_{2}$. 
Knowing this, we can list the possibilities for $L_{i},\left.\left(V /\left[V, Q_{Y}\right]\right)\right|_{L_{i}},\left.\left(V /\left[V, Q_{Y}\right]\right)\right|_{L_{X}^{\prime}}$ based on our inductive knowledge about $L_{X}^{\prime}$ (which is of type $D_{m-1}$ ):

1. $V /\left[V, Q_{Y}\right] \cong$ the natural module for $L_{i}$.

2. ( $\mathrm{U}_{3}$ in Table 2) The natural module $W_{i}$ for $L_{i}$ is reducible as an $L_{X}^{\prime}$-module and different from $V^{1}\left(Q_{Y}\right)=V /\left[V, Q_{Y}\right]$, with $L_{i}$ of type $D_{m},\left.\left(V /\left[V, Q_{Y}\right]\right)\right|_{L_{i}}$ $=\operatorname{spin}\left(L_{i}\right)$, and $\left.\left(V /\left[V, Q_{Y}\right]\right)\right|_{L_{X}^{\prime}}=\operatorname{spin}\left(L_{X}^{\prime}\right) \oplus \operatorname{spin}\left(L_{X}^{\prime}\right)$ (one of the two cases in which $X=D_{m}$ occurs in the situation examined in [4]).

3. ( $\mathrm{U}_{2}$ in Table 2) Same as 2 above, except here $L_{i}$ is of type $B_{m-1}$ and $\left.\left(V /\left[V, Q_{Y}\right]\right)\right|_{L_{i}},\left.\left(V /\left[V, Q_{Y}\right]\right)\right|_{L_{X}^{\prime}}$ are as in the statement of $[4$, Theorem 3.3]. (This is the other case examined in [4].)

4. $W_{i}$ is irreducible for $L_{X}^{\prime}$.

Here is a lemma to simplify things considerably:

Lemma 4.2. In all of the four cases above, if $\alpha \in \Pi(Y)$ and $\left\langle\alpha, \Pi\left(L_{i}\right)\right\rangle=0$, then $\langle\lambda, \alpha\rangle=0$.

Proof. Nodes in $L_{j}$ for $j \neq i$ must have 0 label since $L_{j}$ acts trivially on $V^{1}\left(Q_{Y}\right)$. Assume $\langle\lambda, \alpha\rangle \neq 0$ for some $\alpha \in \Pi(Y)$ with $\left\langle\alpha, \Pi\left(L_{Y}\right)\right\rangle=0$ (i.e. $\alpha$ does not adjoin $\left.\Pi\left(L_{Y}\right)\right)$. Define $K_{Y}^{\alpha}$ as in the discussion following Lemma 2.9. Then $Q_{Y} / K_{Y}^{\alpha}$ has dimension 1 by $[9,3.1]$ (indeed, $\left.Q_{Y} / K_{Y}^{\alpha} \cong U_{-\alpha}\right)$. It has an $L_{X}$-submodule $Q_{X} K_{Y}^{\alpha} / K_{Y}^{\alpha} \cong Q_{X} /\left(Q_{X} \cap K_{Y}^{\alpha}\right)$ by [9, 3.3]. By Lemma 2.10(i), $Q_{X}^{\prime}=K^{\beta_{1}}=\left\langle U_{\beta}\right|$ $\beta \in \Sigma^{-}(X), \beta_{1}$-coefficient of $\left.\beta<-1\right\rangle=1$ (since $D_{m}$ has no roots with a $\beta_{1^{-}}$ coefficient less than -1 ). Then by part (ii) of the same Lemma, $Q_{X}=Q_{X} / K^{\beta_{1}}=$ $Q^{\beta_{1}}$ is an irreducible $L_{X}$-module of high weight $-\left.\beta_{1}\right|_{T_{L_{X}}}$ (and thus dimension $2(m-1))$. But $Q_{X} \cap K_{Y}^{\alpha}$ is a submodule since $K_{Y}^{\alpha}$ is normal in $P_{Y} \geq P_{X} \geq L_{X}$ and since $Z=Z\left(L_{X}\right)^{\circ}$ induces the full group of scalars on $Q_{X}$. So either $Q_{X} \leq K_{Y}^{\alpha}$ or $Q_{X} K_{Y}^{\alpha} / K_{Y}^{\alpha} \cong Q_{X} /\left(Q_{X} \cap K_{Y}^{\alpha}\right) \cong Q_{X} / Q_{X}^{\prime}=Q_{X}$ has dimension $2(m-1)$; the latter is impossible (it has dimension 0 or 1 ). So $\left[V, Q_{Y}\right]=\left[V, Q_{X}\right] \leq\left[V, K_{Y}^{\alpha}\right]$. But the weight space $V_{\lambda-\alpha}$ appears in $\left[V, Q_{Y}\right]$ and not in $\left[V, K_{Y}^{\alpha}\right]$. This is a contradiction, so in fact $\langle\lambda, \alpha\rangle=0$ for $\alpha$ not adjoining $\Pi\left(L_{Y}\right)$.

Now assume $\langle\lambda, \alpha\rangle \neq 0$ for some $\alpha \in \Pi(Y)-\Pi\left(L_{Y}\right)$ such that $\left\langle\alpha, \Pi\left(L_{i}\right)\right\rangle=0$. By the above, there is some $j \neq i$ such that $\left\langle\alpha, \Pi\left(L_{j}\right)\right\rangle \neq 0$. The weight $\lambda-\alpha$ is a weight in $\left[V, Q_{Y}\right]=\left[V, Q_{X}\right]$ but not in $\left[V, K_{Y}^{\alpha}\right]$, so $Q_{X} \leq K_{Y}^{\alpha}$ gives a contradiction as above. So $Q_{X} \not \leq K_{Y}^{\alpha}$. Then $Q_{X} K_{Y}^{\alpha} / K_{Y}^{\alpha} \neq 0$ and as above $Q_{X} K_{Y}^{\alpha} / K_{Y}^{\alpha} \cong$ $Q_{X} /\left(Q_{X} \cap K_{Y}^{\alpha}\right) \cong Q_{X} / K^{\beta_{1}}$ is an irreducible $L_{X}^{\prime}$-module of dimension $2(m-1)$. So $Q_{Y} / K_{Y}^{\alpha} \cong V_{L_{Y}^{\prime}}(-\alpha)$ is an irreducible $L_{Y}^{\prime}$-module of dimension $\geq 2(m-1)$.

Let $\mu=\lambda-\alpha$. Then $\left.\mu\right|_{T_{i}}=\left.\lambda\right|_{T_{i}}$ (where $T_{i}$ is a maximal torus of $L_{i}$ ) and $V_{L_{Y}^{\prime}}(\mu)=V_{L_{i}}(\lambda) \otimes V_{L_{Y}^{\prime}}(-\alpha)$, of dimension $\geq \operatorname{dim}\left(V^{1}\left(Q_{Y}\right)\right)(2 m-2)$. But by Lemma $2.9, \operatorname{dim}\left(V^{2}\left(Q_{Y}\right)\right) \leq \operatorname{dim}\left(V^{1}\left(Q_{Y}\right)\right)(2 m-2)$, and $V_{L_{Y}^{\prime}}(\mu) \leq V^{2}\left(Q_{Y}\right)$. This forces $V_{\alpha}\left(Q_{Y}\right)\left(=V_{L_{Y}}(\mu)\right)=V^{2}\left(Q_{Y}\right)$, but there is some $\epsilon \in \Pi(Y)-\Pi\left(L_{i}\right)$ such that $\left\langle\epsilon, \Pi\left(L_{i}\right)\right\rangle \neq 0$ and $V_{\epsilon}\left(Q_{Y}\right) \neq 0$. From this contradiction, we have that in fact $\langle\lambda, \alpha\rangle=0$ for all $\alpha$ not in or adjoining $\Pi\left(L_{i}\right)$.

We now look at each of the cases 1-4 in turn.

\subsection{Case 1.}

Claim 4.3. $V^{1}\left(Q_{Y}\right) \neq$ the natural module for $L_{i}$, so case 1 on the previous page does not arise.

Proof. Assume $V^{1}\left(Q_{Y}\right)$ is isomorphic to the natural module $W_{i}$ for $L_{i}$. 
There are several possibilities for the type of $L_{i}$. Call the node in $L_{i}$ with a non-zero label $\alpha_{l}$. If $L_{i}$ is of type $B_{k}, C_{k}$, or $D_{k}$, then it corresponds to a subdiagram of the Dynkin diagram for $Y$ at the "end" of that diagram; the picture is

$\stackrel{\gamma}{\alpha_{l}} \stackrel{\alpha_{l}}{\longrightarrow} \ldots{ }^{L_{i}}$

. The only node in $\Pi(Y)$ adjoining $L_{i}$ adjoins $\alpha_{l}$; call it $\gamma$. Now $W_{i} \cong V^{1}\left(Q_{Y}\right)=V_{1}^{1}\left(Q_{X}\right) \oplus V_{2}^{1}\left(Q_{X}\right)$, and these are the only two $L_{X}$-submodules of $V^{1}\left(Q_{Y}\right)$. They are interchanged by $t$. Also, $Q_{Y} / K_{Y}^{\gamma}$ is an $L_{Y}^{\prime}$-module of high weight $-\gamma ; Q_{X} K_{Y}^{\gamma} / K_{Y}^{\gamma}$ is a non-zero $t$-stable $L_{X}$-submodule $\left(Q_{X} \leq K_{Y}^{\gamma}\right.$ gives a contradiction as in the proof of the last lemma). But we just said that the natural module for $L_{i}$ has no $t$-stable $L_{X}$-submodules; this forces there to be an $L_{j}$ adjoining $\gamma$ such that $L_{X}^{\prime}$ projects non-trivially to $L_{j}$. Thus the natural module for $L_{j}$ has dimension $\geq 2(m-1)$. But then if $\gamma$ has non-zero label, we have a composition factor of high weight $\left.(\lambda-\gamma)\right|_{T_{L_{Y}^{\prime}}}$ in $V^{2}\left(Q_{Y}\right)$, of dimension $>2(m-1) \operatorname{dim}\left(V^{1}\left(Q_{Y}\right)\right)$. And if $\gamma$ has label 0 , we have $\lambda-\gamma-\alpha_{l}$, also giving dimension $>2(m-1) \operatorname{dim}\left(V^{1}\left(Q_{Y}\right)\right)$. By Lemma 2.9, this is a contradiction. Note that this same argument holds whenever there is a node in $\Pi(Y)-\Pi\left(L_{i}\right)$ adjoining $\alpha_{l}$ via a single bond. So $L_{i}$ must be of type $A_{k}$, with no node outside $\Pi\left(L_{i}\right)$ adjoining $\alpha_{l}$ via a single bond.

By Lemma 4.3, all nodes in $\Pi(Y)$ not adjoining $\Pi\left(L_{i}\right)$ have marking 0 . There is no node outside $\Pi\left(L_{i}\right)$ adjoining $\alpha_{l}$ via a single bond. The other possibilities for nodes adjoining $L_{i}$ are the following, which we will consider in turn:
a) $\begin{gathered}L_{i} \\ \alpha_{l} \gamma\end{gathered}$
b) $\begin{gathered}L_{i} \\ \alpha_{l} \gamma\end{gathered}$

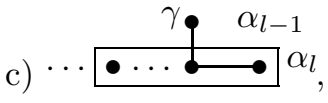
d)

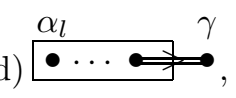
e)
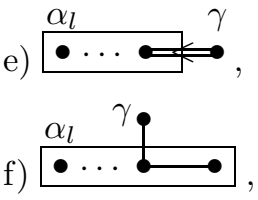
g) $\stackrel{\alpha_{l}}{\bullet \cdots \bullet \bullet \cdots . .}$

a) $Q_{Y} / K_{Y}^{\gamma} \cong V^{1}\left(Q_{Y}\right)$; as above, this forces there to be an $L_{j}$ adjoining $\gamma$, which is absurd.

b) The rank of $L_{i}$ is $k$, so $\operatorname{dim}\left(V^{1}\left(Q_{Y}\right)\right)=k+1$. We then have $k+1=$ $\operatorname{dim}\left(V^{1}\left(Q_{Y}\right)\right) \geq 2^{m-1}>\operatorname{dim}\left(Q^{\beta_{1}}\right)$. (The dimension of $V^{1}\left(Q_{Y}\right)$ is at least $2^{m-1}$ since the high weight of $V_{1}^{1}\left(Q_{X}\right)$ is not symmetric with respect to the graph automorphism of $D_{m-1}$; thus the Weyl group orbit of the $T_{L_{X}^{\prime}}$-high weight of $V_{1}^{1}\left(Q_{X}\right)$ contains at least $2^{m-2}$ weights.) In particular, $k+1 \geq 8$ since $m \geq 4$. Note also that $\operatorname{dim}\left(V^{1}\left(Q_{Y}\right)\right)$ is even (so $k$ is odd) because it is the sum of two $L_{X}^{\prime}$-modules of equal dimension. If $\gamma$ has a non-zero label in the marking for $\lambda$ on $Y$, then $V^{2}\left(Q_{Y}\right)$ has a composition factor with high weight $\left.(\lambda-\gamma)\right|_{T_{L_{Y}^{\prime}}}$, giving $\operatorname{dim}\left(V^{2}\left(Q_{Y}\right)\right) \geq$ 
$\operatorname{dim}\left(V_{L_{i}}\left(\left.(\lambda-\gamma)\right|_{T_{Y}}\right)\right)=\frac{1}{6}\left(\operatorname{dim}\left(V^{1}\left(Q_{Y}\right)\right)+2\right)\left(\operatorname{dim}\left(V^{1}\left(Q_{Y}\right)\right)+1\right)\left(\operatorname{dim}\left(V^{1}\left(Q_{Y}\right)\right)\right)>$ $2(m-1) \operatorname{dim}\left(V^{1}\left(Q_{Y}\right)\right)$, which is a contradiction to Lemma 2.9. If $\gamma$ has label 0 , then the high weight $\left.\left(\lambda-\alpha_{l}-\gamma\right)\right|_{T_{L_{Y}^{\prime}}}=\left.\left(\lambda_{l-1}+\lambda_{l}\right)\right|_{T_{L_{Y}^{\prime}}}$ appears. This weight has $k(k+1)$ conjugates, and the subdominant weight $\left(\lambda_{l-2}\right)$ has $\frac{(k+1) !}{6(k-2) !}$ conjugates. But for $k>6, k(k+1)+\frac{(k+1) !}{6(k-2) !}>(k+1)^{2}>2(m-1) \operatorname{dim}\left(V^{1}\left(Q_{Y}\right)\right)$, again a contradiction. So b) does not arise.

c) Take $k$ as in b). If $\gamma$ has non-zero marking, then $V^{2}\left(Q_{Y}\right)$ has a composition factor of high weight $\left.(\lambda-\gamma)\right|_{T_{L_{Y}^{\prime}}}=\left.\left(\lambda_{l-1}+\lambda_{l}\right)\right|_{T_{L_{Y}^{\prime}}}$, and we have a contradiction as above. If $\gamma$ has marking 0 , then we have the high weight $\left.\left(\lambda-\alpha_{l}-\alpha_{l-1}-\gamma\right)\right|_{T_{L_{Y}^{\prime}}}=$ $\left.\lambda_{l-2}\right|_{T_{L^{\prime}}}$, which has, as above, $\frac{(k+1) k(k-1)}{6}$ conjugates. For $k \geq 8, \frac{(k+1) k(k-1)}{6}>$ $(k+1)^{2} \geq 2(m-1) \operatorname{dim}\left(V^{1}\left(Q_{Y}\right)\right)$; this is a contradiction to the same result. If $k=7$, then $\operatorname{dim}\left(V_{L_{i}}\left(\lambda_{l-2}\right)\right)=\left(\begin{array}{l}8 \\ 3\end{array}\right)=56>6 \cdot 8=\operatorname{dim}\left(Q^{\beta_{1}}\right) \operatorname{dim}\left(V^{1}\left(Q_{Y}\right)\right)$, and again we have a contradiction.

d)-g) Let $k+1=\operatorname{dim}\left(V^{1}\left(Q_{Y}\right)\right)$ as above. In any of these cases, if $\gamma$ has a non-zero label, then $V^{2}\left(Q_{Y}\right)$ has a composition factor (given by the high weight $\left.(\lambda-\gamma)\right|_{T_{L_{Y}^{\prime}}}$ ) of dimension greater than $\operatorname{dim}\left(Q^{\beta_{1}}\right) \operatorname{dim}\left(V^{1}\left(Q_{Y}\right)\right)$, giving a contradiction as above. If $\gamma$ has a zero label, then $V \cong$ the natural module $W$ for $Y$, which is impossible, since $X$ acts irreducibly on $W$ but not on $V$.

\subsection{Case 2.}

Claim 4.4. Case 2 (stated above Lemma 4.2) does not arise.

Proof. We have the picture below, with the boxed diagrams on the left of type $D_{m-1}$ and on the right of type $D_{m}$ :

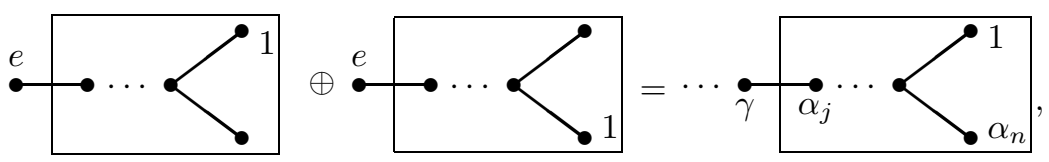

with $j=n-m+1$ ( $n$ is the $\operatorname{rank}$ of $Y$, as always). If $\gamma$ has a non-zero label, then in the characteristic 0 case,

$$
\begin{gathered}
\operatorname{dim}\left(V^{2}\left(Q_{Y}\right)\right) \geq \operatorname{dim}\left(V_{\gamma}\left(Q_{Y}\right)\right) \geq \operatorname{dim}\left(V_{D_{m}}\left(\lambda_{j}+\lambda_{n-1}\right)\right) \\
=(2 m-1) \operatorname{dim}\left(V^{1}\left(Q_{Y}\right)\right)>\operatorname{dim}\left(Q^{\beta_{1}}\right) \operatorname{dim}\left(V^{1}\left(Q_{Y}\right)\right),
\end{gathered}
$$

again a contradiction to Lemma 2.9. The only problem here is when the irreducible module $V_{D_{m}}\left(\lambda_{j}+\lambda_{n-1}\right)$ is not the Weyl module.

We can use the Andersen-Jantzen sum formula to check that in those characteristics for which the Weyl module does reduce, it reduces only by $2^{m-1}$, making the bound sharp: In these cases, $\operatorname{dim}\left(V_{D_{m}}\left(\lambda_{j}+\lambda_{n-1}\right)\right)=(2 m-2) \operatorname{dim}\left(V^{1}\left(Q_{Y}\right)\right)$. So if there is anything else in $V_{\gamma}\left(Q_{Y}\right), V^{2}\left(Q_{Y}\right)$ will again be too big. So assume the $D_{m}$-module $V^{1}$ with high weight marking $\bullet \cdots \cdots$ weight $\lambda_{j}+\lambda_{n-1}=\lambda^{\prime}$ ) does reduce. Then the dimension of the weight space $V_{\lambda^{\prime}-\alpha_{j}-\cdots-\alpha_{n-1}}^{1}$ in $V\left(\lambda^{\prime}\right)$ is $m-2$ (this dimension is $m-1$ in the characteristic 0 
case). But in $V$, which has marking

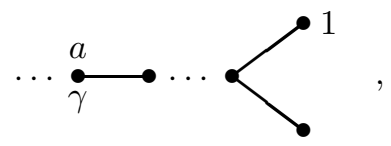

the dimension of the weight space for the weight $\lambda-\gamma-\alpha_{j}-\cdots-\alpha_{n-1}$ is $m$ or $m-1$ and this weight space is in $V_{\gamma}\left(Q_{Y}\right)$. So if $V\left(\lambda^{\prime}\right)$ reduces, then there is something else in $V_{\gamma}\left(Q_{Y}\right)$, and again we get that $V^{2}\left(Q_{Y}\right)$ is too large. So in fact $\gamma$ has label 0 (i.e. $\langle\lambda, \gamma\rangle=0$ ), and, since we have already shown that nodes not adjoining $L_{i}$ have 0 label, $V$ is the spin module for $Y$.

Now look at a different parabolic subgroup of $X$ : Let $P_{X}=L_{X} Q_{X}$ correspond to $\Pi(X)-\left\{\beta_{m-1}, \beta_{m}\right\}$. Use the standard notation for a basis of the Lie algebra of $X$ : For a simple Lie algebra with root system $\Phi$ having basis $\left\{\alpha_{1}, \ldots, \alpha_{m}\right\}$, use the Chevalley basis $\left\{e_{\alpha}, f_{\alpha}, h_{i} \mid \alpha \in \Phi^{+}, 1 \leq i \leq m\right\}$, satisfying the usual relations - in particular, $\left[e_{\alpha_{i}} f_{\alpha_{i}}\right]=h_{i}$. Then each $V_{i}(i=1,2)$ is spanned by vectors of the form $w=f_{\alpha} \ldots f_{\beta} v_{i}^{+}$, where $v_{i}^{+}$is a maximal vector. Order the roots in $\Sigma(X)$ so that the last $f_{\gamma}$ applied correspond to roots in $\Sigma(X)-\Sigma\left(L_{X}^{\prime}\right)$. Now $V_{i}^{2}\left(Q_{X}\right)$ is spanned by vectors $f_{\alpha} w$ where $w=f_{\beta} \ldots f_{\epsilon} v_{i}^{+}$such that all the roots $\beta, \ldots, \epsilon$ are in $\Sigma\left(L_{X}^{\prime}\right)$ and $\alpha$ has $Q_{X}$-level 1 (i.e. $\alpha$ has $\beta_{m-1^{-}}$or $\beta_{m}$-coefficient -1 and the other, 0 ). If we take a maximal linearly independent set of such $w$, we have a basis of $V_{i}^{1}\left(Q_{X}\right)$, and there are $2(m-1)$ roots $\alpha$ of $Q_{X}$-level 1 . So $\operatorname{dim}\left(V_{i}^{2}\left(Q_{X}\right)\right) \leq 2(m-1) \operatorname{dim}\left(V_{i}^{1}\left(Q_{X}\right)\right)$. This then gives $\operatorname{dim}\left(V^{2}\left(Q_{Y}\right)\right) \leq 2 \operatorname{dim}\left(V_{1}^{2}\left(Q_{X}\right)\right) \leq 2\left(2(m-1) \operatorname{dim}\left(V_{1}^{1}\left(Q_{X}\right)\right)\right)=$ $2(m-1) \operatorname{dim}\left(V^{1}\left(Q_{Y}\right)\right)$.

If $e=0$ (with $e$ the labelling on $\beta_{1}$ as in the picture at the beginning of the proof), then $\left.V\right|_{X}$ is a sum of two spin modules. So $\operatorname{dim}(V)=2\left(2^{m-1}\right)=2^{m}$. Since $V$ is a spin module for $Y, \operatorname{dim}(V)=2^{n-1}$. So $X=D_{n-1}$. But the natural module for $Y$ has dimension $2 n$, and $D_{n-1}$ for $n \geq 5$ has no irreducible restricted modules of this dimension. So $e \neq 0$.

$V$ is the spin module for $Y$, and the parabolic subgroup $P_{Y}$ of $Y$ in which we embed this new $P_{X}$ must contain both of the root groups corresponding to the node with a label of 1 in the marking for the $Y$-high weight of $V$ (since $e \neq 0$ ). Since this 1 is the only non-zero label in the marking, the $L_{i}$ which contains it is the only $L_{j}$ acting non-trivially on $V /\left[V, Q_{Y}\right]$. Now $L_{X}^{\prime}$ is of type $A_{m-2}$, and $V_{1} /\left[V_{1}, Q_{X}\right]$ has high weight $e \delta_{1}$.

There are two possibilities for the type of $L_{i}: D_{l+1}$ for some $l \geq 3$ and $A_{l}$. If $L_{i}$ is of type $D_{l+1}$, then $\operatorname{dim}\left(V /\left[V, Q_{Y}\right]\right)=2^{l}$ is a power of 2. By Lemma 2.5, $\operatorname{dim}\left(V_{1} /\left[V_{1}, Q_{X}\right]\right)=\left(\begin{array}{c}m-2+e \\ e\end{array}\right)$, which is not a power of two unless $e=1$ or $m=3$. But $m \geq 4$, so this forces $e=1$. Then $\operatorname{dim}\left(V /\left[V, Q_{Y}\right]\right)=(m-2)+1=2^{l}$. A group of type $D_{l+1}$ for $l \geq 3$ does not contain a group of type $A_{2^{l}-1}$, however, so $L_{i}$ is not of type $D_{l+1}$ for $l \geq 3$.

If $L_{i}$ is of type $A_{l}$, then by Lemma 2.5 we have $l+1=2 \operatorname{dim}\left(V_{1} /\left[V_{1}, Q_{X}\right]\right)=$ $2\left(\begin{array}{c}m-2+e \\ e\end{array}\right)>4$. The root $\alpha_{n}$ (see the picture at the beginning of the proof) is not contained in the set of roots corresponding to $L_{Y}$ (since $l>3$ ), so $V^{2}\left(Q_{Y}\right)$ contains a composition factor of high weight $\left.\left(\lambda-\alpha_{n-1}-\alpha_{n-2}-\alpha_{n}\right)\right|_{T_{Y}}$, of dimension $\left(\begin{array}{c}l+1 \\ 3\end{array}\right)$. Then we have $\frac{1}{6}(l+1) l(l-1) \leq \operatorname{dim}\left(V^{2}\left(Q_{Y}\right)\right) \leq 2(m-1) \operatorname{dim}\left(V^{1}\left(Q_{Y}\right)\right)=$ $2(m-1)(l+1)$, with $l$ given in terms of $m$ and $e$ above. This is impossible in all 
cases except $m=4, e=1$ and $m=5, e=1$. So the only two possibilities left here

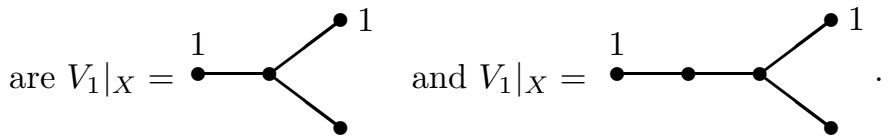

Remember that $V$ is the spin module for $Y$, so has dimension a power of 2. So $V_{1}$ has dimension a power of 2 . The only time when one of the above two modules has dimension a power of 2 is the $D_{5}$ module of high weight $\delta_{1}+\delta_{4}$ in characteristic 5 , of dimension $2^{7}$. So $\operatorname{dim}(V)=2^{8}$ and $Y$ is of type $D_{9}$. But $D_{5}$ has no 18-dimensional irreducible representations in characteristic 5 . So $L_{i}$ is not of type $A_{l}$.

\subsection{Case 3.}

Claim 4.5. The situation outlined in case 3 (stated above Lemma 4.2) does not arise.

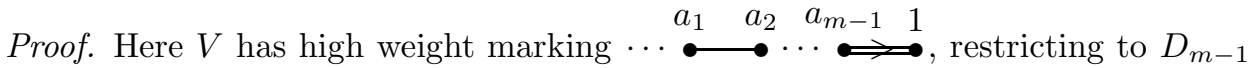
and with $a_{2}, \ldots, a_{m}=1$ related as in [4,3.3], and with labels to the left of $a_{1}$ all 0 . Let $P_{X}$ be the parabolic subgroup of $X$ corresponding to $\Pi(X)-\left\{\beta_{m}\right\}$. As always, embed $P_{X}$ in a parabolic subgroup $P_{Y}$ of $Y$ via the construction given in the introduction, so that $Q_{X} \leq Q_{Y}$ and $L_{X} \leq L_{Y}$.

As in the proof of Lemma 3.5, we show that in this case $V /\left[V, Q_{Y}\right]$ is irreducible as an $L_{X}$-module. Let $Z=Z\left(L_{X}\right)^{\circ}$. By construction, $Z \leq Z\left(L_{Y}\right)$ (where $L_{Y}=$ $\left.C_{Y}(Z)\right)$, so $Z$ induces scalars on $V /\left[V, Q_{Y}\right]$ (since $L_{Y}$ acts irreducibly). But if $V /\left[V, Q_{Y}\right]$ is not irreducible for $L_{X}$, then $V /\left[V, Q_{Y}\right]=V_{1} /\left[V_{1}, Q_{X}\right] \oplus V_{2} /\left[V_{2}, Q_{X}\right]$, and $Z$ acts differently on these two $L_{X}$-modules:

$$
\begin{aligned}
Z & =\left\{\operatorname{diag}\left(a, \ldots, a, a^{-1}, \ldots, a^{-1}\right) \mid a \in K^{*}\right\} \\
& =\left\{\operatorname{diag}\left(a^{2}, \ldots, a^{2}, a^{-2}, \ldots, a^{-2}\right) \mid a \in K^{*}\right\} \\
& =\left\{h_{\beta_{1}}\left(a^{2}\right) h_{\beta_{2}}\left(a^{4}\right) \ldots h_{\beta_{m-2}}\left(a^{2(m-2)}\right) h_{\beta_{m-1}}\left(a^{m-2}\right) h_{\beta_{m}}\left(a^{m}\right) \mid a \in K^{*}\right\} .
\end{aligned}
$$

The two $X$-modules $V_{1}, V_{2}$ have high weight labelling
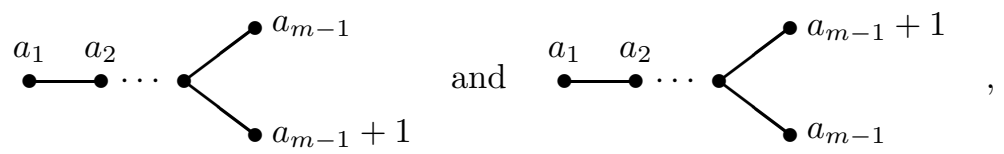

so $h_{\beta_{1}}\left(a^{2}\right) h_{\beta_{2}}\left(a^{4}\right) \ldots h_{\beta_{m-2}}\left(a^{2(m-2)}\right) h_{\beta_{m-1}}\left(a^{m-2}\right) h_{\beta_{m}}\left(a^{m}\right)$ acts differently on a high weight vector $v_{1} \in V_{1}$ than on a high weight vector $v_{2} \in V_{2}$. Since $v_{i}$ has a nonzero image in $V_{i} /\left[V_{i}, Q_{X}\right]$, this shows that only one of the $V_{i} /\left[V_{i}, Q_{X}\right]$ can be in $V /\left[V, Q_{Y}\right]$. So $V /\left[V, Q_{Y}\right]$ is irreducible as an $L_{X}$-module. Assume $V_{1}$ is the summand which projects non-trivially to $V /\left[V, Q_{Y}\right]$ (so $V_{2} \subseteq\left[V, Q_{Y}\right]$ ).

Irreducible restricted $A_{m-2}$-modules are tensor indecomposable (Lemma 2.1), so only one of the simple factors $L_{i}$ of $L_{Y}^{\prime}$ acts non-trivially on $V /\left[V, Q_{Y}\right]=V^{1}\left(Q_{Y}\right)$. The two possibilities for the type of $L_{i}$ are $B_{k}$ and $A_{k}$. If $L_{i}$ is of type $B_{k}$, then $V^{1}\left(Q_{Y}\right) \cong$ the natural module for $L_{i}$, since there are no overgroups of $A_{l}$ of type $B_{k}$ appearing in [9, Table 1]. But we know $a_{m}=1$, so the only possibility here is $L_{i}$ of type $B_{2} \cong C_{2}$, and $V^{1}\left(Q_{Y}\right) \cong$ the natural module for $C_{2}$, of dimension 4 . Then $m=4, L_{X}^{\prime}=A_{3}, X=D_{4}$, and $V_{1}^{1}\left(Q_{X}\right)$ is of $L_{X}$-high weight $\delta_{3}$ or $\delta_{1}$. If $\delta_{3}$, then the picture is 


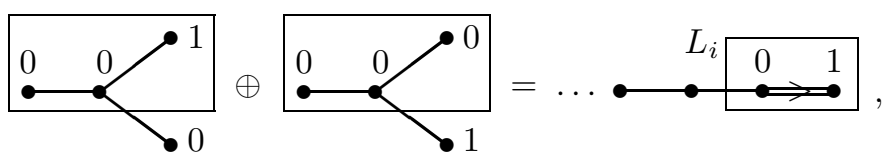

so $\operatorname{dim}(V)=\operatorname{dim}\left(V_{1}\right)+\operatorname{dim}\left(V_{2}\right)=16$. Since $a_{m}=1$, the $T_{Y}$-high weight of $V$ has at least $|\mathcal{W}| /\left|\mathcal{W}_{A_{n-1}}\right|=2^{n} n ! / n !=2^{n}$ conjugates, where $\mathcal{W}$ is the Weyl group of type $B_{n}$ and $\mathcal{W}_{A_{n-1}}$ is that of type $A_{n-1}$. So $n \leq 4$; since $4=m \leq n$, we have $Y=B_{4}$. But then if $p \neq 2, \operatorname{dim}(W)=9$, and $D_{4}$ has no irreducible representations of dimension 9. If $p=2$, then we have one of the situations studied already in [4], which does give the example listed in the theorem.

If the $T_{L_{X}^{\prime}}$-high weight of $V_{1} /\left[V_{1}, Q_{X}\right]$ is $\delta_{1}$, then the picture is

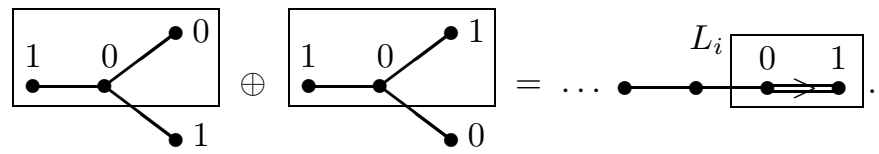

In this case, $\operatorname{since} \operatorname{dim}(V)=2 \operatorname{dim}\left(V_{1}\right)$, we have $\operatorname{dim}(V)=112$ if $p \neq 2, \operatorname{dim}(V)=96$ if $p=2$. But $Y$ cannot have type $B_{4}$ unless $p=2$ (in which case [4] tells us there are no examples of this type) as above, and no irreducible restricted $B_{l}$-module, for $l \geq 5$, whose high weight has an $\lambda_{l}$-coefficient of 1 has dimension 112 or 96 . So $L_{i}$ is not of type $B_{k}$.

So $L_{i}$ is of type $A_{k}$. The arguments in Lemma 4.3 showing that nodes not adjoining $L_{i}$ have marking 0 fail here, since $\left[V, Q_{Y}\right] \neq\left[V, Q_{X}\right]$. So we need new arguments. Assume $\gamma$ is a node in $\Pi(Y)$ which has non-zero label and does not adjoin $\Pi\left(L_{Y}\right)$. The argument in the proof of Lemma 4.2 that $Q_{X} \leq K_{Y}^{\gamma}$ is still valid here, so $V_{\lambda-\gamma} \notin\left[V, Q_{X}\right]$. We have $V /\left[V, Q_{Y}\right]=\left(V_{1} \oplus V_{2}\right) /\left(V_{2} \oplus\left[V, Q_{X}\right]\right)$, so if we can show that $V_{\lambda-\gamma} \notin V_{2}$ we will have a contradiction, since $V_{\lambda-\gamma} \in\left[V, Q_{Y}\right]$.

Since $\left\langle\gamma, \Pi\left(L_{Y}\right)\right\rangle=0$, we know that $\left.\lambda\right|_{T_{L_{Y}^{\prime}}}=\left.(\lambda-\gamma)\right|_{T_{L_{Y}^{\prime}}}$, and thus $\left.\lambda\right|_{T_{L_{X}^{\prime}}}=$ $\left.(\lambda-\gamma)\right|_{T_{L_{X}^{\prime}}}$. If $\gamma_{i}$ is the $T_{X}$-high weight of $V_{i}$, then $\left.\lambda\right|_{T_{X}}=\gamma_{i}$ for $i=1$ or 2 ; we have the pictures

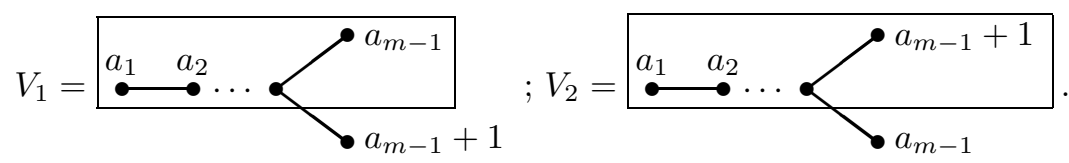

Assume $\left.\lambda\right|_{T_{X}}=\gamma_{1}$. Then $\left.\gamma_{1}\right|_{T_{L_{X}^{\prime}}}=\left.\lambda\right|_{T_{L_{X}^{\prime}}}=\left.(\lambda-\gamma)\right|_{T_{L_{X}^{\prime}}}$ is the $T_{L_{X}^{\prime}}$-high weight of $V_{1} /\left[V_{1}, Q_{X}\right]$. Then to have the weight $\left.(\lambda-\gamma)\right|_{T_{X}}$ in $V_{2}$ would imply we could subtract roots from $\gamma_{2}$ and obtain a weight $\epsilon$ which has the same labelling as $\gamma_{1}$ on $\Pi\left(L_{X}\right)$. Say we subtract $c \beta_{1}$. To balance this (the labelling on $\beta_{1}$ is the same for $\gamma_{1}$ as for $\left.\gamma_{2}\right)$, we must subtract $2 c \beta_{2}, 3 c \beta_{3}, \ldots,(m-2) c \beta_{m-2}$. Say we subtract $d \beta_{m-1}$ and $e \beta_{m}$. To balance the $\delta_{m-2}$ coefficient, we need $2(m-2) c-(m-3) c-d-e=0$. To get $a_{m-1}$ as the $\delta_{m-1}$ coefficient, we need $2 d-(m-2) c=1$. These give $e=(m-1) c-d$ and $d=((m-2) c+1) / 2$.

Now $\left[9,3.6\right.$ iv)] gives $\left.\gamma\right|_{Z}=\left.\beta_{m}\right|_{Z}$. So since $Z \leq T_{X}$ and $\left.(\lambda-\gamma)\right|_{T_{X}}=\left.\left(\gamma_{1}-\gamma\right)\right|_{T_{X}}$, we have $\left.\epsilon\right|_{Z}=\left.(\lambda-\gamma)\right|_{Z}=\left.\left(\gamma_{1}-\gamma\right)\right|_{Z}=\left.\left(\gamma_{1}-\beta_{m}\right)\right|_{Z}$. We computed $Z$ above and

$$
\left(\gamma_{1}-\beta_{m}\right)\left(h_{\beta_{1}}\left(a^{2}\right) h_{\beta_{2}}\left(a^{4}\right) \ldots h_{\beta_{m-2}}\left(a^{2(m-2)}\right) h_{\beta_{m-1}}\left(a^{m-2}\right) h_{\beta_{m}}\left(a^{m}\right)\right)=a^{A-4}
$$


where $A=2 a_{1}+4 a_{2}+\cdots+2 a_{m-2}(m-2)+a_{m-1}(m-2)+\left(a_{m-1}+1\right) m$. So $\epsilon$ must act on $Z$ as $a^{A-4}$. Let the $\delta_{m}$-coefficient of $\epsilon$ be $a_{m}^{\prime}$; we know all the other coefficients of $\epsilon$ (they are $\left.a_{1}, \ldots, a_{m-1}\right)$. Since $\epsilon$ must act as $a^{A-4}$ on the element of $Z$ given above, we have $A-4=2 a_{1}+4 a_{2}+\cdots+2 a_{m-2}(m-2)+a_{m-1}(m-2)+a_{m}^{\prime} m$, or $a_{m}^{\prime} m=\left(a_{m-1}+1\right) m-4$. But $a_{m}^{\prime}=a_{m-1}-2 e+(m-2) c\left(\right.$ we subtracted $(m-1) c \beta_{m-2}$ and $\left.e \beta_{m}\right)$. So $1-(4 / m)=-2 e+(m-2) c=2 c(m-2)-2 c(m-1)+1$. This gives $4 / m=2 c$, or $2 / m=c$. But $m \geq 4$, so $2 / m=c$ has no integer solutions. This is a contradiction.

If $\left.\lambda\right|_{T_{X}}=\gamma_{2}$, similar computations also give $2 / m=c$ and a contradiction as above. So all nodes with non-zero labels must be in $\Pi\left(L_{i}\right)$ or adjoin $\Pi\left(L_{Y}\right)$.

Now let $\gamma=\alpha_{n}$ be the "end" node of the Dynkin diagram for $Y$, which has type $B_{n}$. We know $\alpha_{n}$ has a label of 1 (and $\alpha_{n} \notin \Pi\left(L_{i}\right)$ because $L_{i}$ does not have type $\left.B_{l}\right)$ so, by the above, $\alpha_{n}$ adjoins some $\Pi\left(L_{j}\right)$. If $j \neq i$, then we may assume $L_{X}$ projects non-trivially to $L_{j}$ (else we can take $P_{Y}$ such that $L_{Y}^{\prime}=L_{1} \times \cdots \times \widehat{L_{j}} \times \ldots$ and still have a parabolic satisfying all the hypotheses we used above; then $\alpha_{n}$ would not adjoin $\Pi\left(L_{Y}\right)$ and the above would give a contradiction). So $L_{j}$ has rank at least $m-1$, but then all labels to the left of $L_{j}$ are 0 and there is no room for $L_{i}$, which acts non-trivially on $V /\left[V, Q_{Y}\right]$. So in fact $\alpha_{n}$ adjoins $\Pi\left(L_{i}\right)$. So the picture

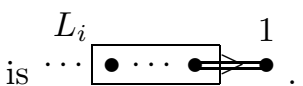

The possibilities are $L_{X}^{\prime}, L_{Y}^{\prime}, V /\left[V, Q_{Y}\right]$ which appear in Table 1 of $[9] ; V /\left[V, Q_{Y}\right]$ is the natural module for $L_{i}$; or $L_{i}$ is of type $A_{m-1}$, with the embedding $L_{X}^{\prime} \hookrightarrow L_{i}$ an isomorphism. There are only 2 possibilities arising from Table 1 of [9]; they give easy contradictions to the fact that most of the (possibly) non-zero labels for the $T_{X}$-high weights of $V$ coincide with the corresponding labels for the $T_{Y}$-high weight of $V$.

Assume that $V^{1}\left(Q_{Y}\right)$ is isomorphic to the natural module for $L_{i}$. Let $k$ be the rank of $L_{i}$. If the picture is
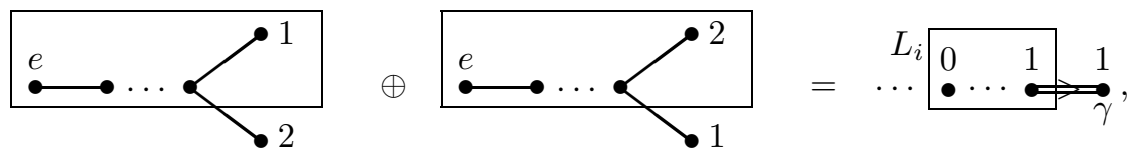

then we know that $p=5$ (as this is the only characteristic in which this configuration arises in $[4,3.3]$ ) and that there are no non-zero labels to the left of $L_{i}$ (since at most the end $m$ labels are non-zero as mentioned at the beginning of the proof, and $k>m$ ). Let $V_{1}$ be the $L_{X}$-module which projects non-trivially to $V /\left[V, Q_{Y}\right]$ (not necessarily the first summand pictured above). Here $Q_{X} \leq K_{Y}^{\alpha_{n}}$ (since otherwise $Q_{Y} / K_{Y}^{\alpha_{n}} \cong V /\left[V, Q_{Y}\right]$ would have an $L_{X}$-submodule $Q_{X} K_{Y}^{\alpha_{n}} / K_{Y}^{\alpha_{n}} \cong Q_{X} / K^{\beta_{m}}$, which it does not since $V /\left[V, Q_{Y}\right]$ is irreducible for $L_{X}^{\prime}$ ), so again we will have a contradiction if we can show that $V_{\lambda-\alpha_{n}} \nsubseteq V_{2}$, since $\left[V, Q_{Y}\right]=\left[V_{1}, Q_{X}\right]+V_{2}$, $V_{\lambda-\alpha_{n}} \nsubseteq\left[V_{1}, Q_{X}\right]$, and $V_{\lambda-\alpha_{n}} \subseteq\left[V, Q_{Y}\right]$.

Call the two $T_{X}$-high weights pictured above $\mu$ and $\nu$ respectively. If $e=0$, we know from the main result of [4] that $\operatorname{dim}\left(V_{1}\right)+\operatorname{dim}\left(V_{2}\right)$ is the dimension of the $B_{m}$-module (where $m=$ the rank of $X=D_{m}$ ) with high weight $\lambda_{m-1}+\lambda_{m}$. So if $n(=$ the rank of $Y)$ is greater than $m$, the dimension of $V$ is strictly larger than $\operatorname{dim}\left(V_{1}\right)+\operatorname{dim}\left(V_{2}\right)$, which is a contradiction. So if $e=0$, then $n=m$ and we must be in the $D_{n}<B_{n}$ case. Assume $e>0$. We have $\left.\left(\lambda-\alpha_{n}\right)\right|_{T_{L_{Y}^{\prime}}}=$ 
$\left.\lambda\right|_{T_{L_{Y}^{\prime}}}+\left.\lambda_{n-1}\right|_{T_{L_{Y}^{\prime}}}=\left.2 \lambda\right|_{T_{L_{Y}^{\prime}}}$. Assume $\left.\lambda\right|_{T_{X}}=\mu$ (i.e. the first summand pictured above is the one that projects non-trivially to $\left.V /\left[V, Q_{Y}\right]\right)$. Then $\left.\mu\right|_{T_{L_{X}^{\prime}}}=\left.\lambda\right|_{T_{L_{X}^{\prime}}}$ is the $T_{L_{X}^{\prime}}$-high weight of $V /\left[V, Q_{Y}\right]$. To have the weight $\left.\left(\lambda-\alpha_{n}\right)\right|_{T_{X}}$ in $V_{2}$ would mean we could subtract roots from $\nu$ and obtain a weight $\epsilon$ which restricts to $T_{L_{X}^{\prime}}$ in the same way as $2 \lambda$ does; i.e. there must be integers $c, \ldots, d, f$ such that $2 e \delta_{1}+2 \delta_{m-1}+a \delta_{m}=e \delta_{1}+2 \delta_{m-1}+\delta_{m}-\left(c \beta_{1}+\cdots+d \beta_{m-1}+f \beta_{m}\right)$ for some $a$. If we subtract $c \beta_{1}$, we must subtract $(2 c+e) \beta_{2},(3 c+2 e) \beta_{3}, \ldots,((m-2) c+(m-3) e) \beta_{m-2}$. Say we subtract $d \beta_{m-1}$ and $f \beta_{m}$. Then since the coefficients of $\delta_{m-2}, \delta_{m-1}$ remain unchanged, we have $(m-3) c+(m-4) e+d+f=2((m-2) c+(m-3) e)$ and $(m-2) c+(m-3) e=2 d$. These together give $f=(m(c+e)-e) / 2$

Lemma 3.6 iv) in [9] gives $\left.\alpha_{n}\right|_{Z}=\left.\beta_{m}\right|_{Z}$. So $\left.\left(\lambda-\alpha_{n}\right)\right|_{Z}=\left.\left(\mu-\beta_{m}\right)\right|_{Z}$. We computed $Z$ above, and

$$
\begin{gathered}
\left(\mu-\beta_{m}\right)\left(h_{\beta_{1}}\left(a^{2}\right) h_{\beta_{2}}\left(a^{4}\right) \ldots h_{\beta_{m-2}}\left(a^{2(m-2)}\right) h_{\beta_{m-1}}\left(a^{m-2}\right) h_{\beta_{m}}\left(a^{m}\right)\right) \\
=a^{2 e+(m-2)+2 m-4} .
\end{gathered}
$$

So $\epsilon$ must act on $Z$ as $a^{2 e+(m-2)+2 m-4}$. But $2 \lambda$ acts as

$$
a^{4 e+2(m-2)+(1-2 f+(m-2) c+(m-3) e) m} .
$$

So these two exponents must be equal. Using the above expression for $f$, this simplifies to $m(e+c)=e+2$, which has no solutions in non-negative integers with $m \geq 4$. So $\left.\lambda\right|_{T_{X}} \neq \mu$.

If $\left.\lambda\right|_{T_{X}}=\nu$, then similar calculations give $2 f=m(c+e)-e-3$ and $m(e+c-3)=$ $e$; this system also has no solutions in non-negative integers with $m \geq 4, e \geq 1$.

So the picture above does not occur.

If the non-zero label is on the other end of $L_{i}$, then the picture is
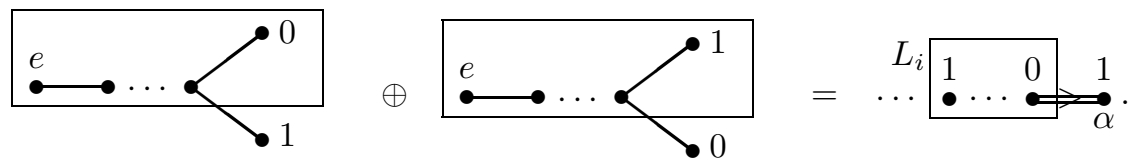

The rank of $L_{i}$ is $\operatorname{dim}\left(V^{1}\left(Q_{Y}\right)\right)-1$; we know that the node in the Dynkin diagram for $Y$ with a label of 1 must be within $m$ nodes of the end (it must be $\alpha_{i}$ for $i \geq n-m)$, as noted at the beginning of this proof. If the second summand in the picture is the one that projects non-trivially to $V^{1}\left(Q_{Y}\right)$, this forces $e=0$ but then $V$ has dimension too large to be the sum of the two spin modules for $D_{m}$. If the first summand projects non-trivially, $e=1$ for the same reason and $L_{i}$ has rank $m-1$.

If there is a node to the left of $L_{i}$ (i.e. if $n>m$ ), let $P_{Y}$ be the parabolic subgroup of $Y$ corresponding to the end $m$ nodes of the Dynkin diagram. Then $\left.V\right|_{L_{Y}^{\prime}}$ has a composition factor (not all of $V$ ) which is isomorphic to the $B_{m}$ module with high weight $\lambda_{1}+\lambda_{m}$. This $B_{m}$-module has $D_{m}$-high weights $\delta_{1}+\delta_{m-1}$ and $\delta_{1}+\delta_{m}$ (for the $D_{m} \leq L_{Y}^{\prime}$ in the natural way); thus $V$ is too large to be the sum of two $D_{m}$-modules of these high weights. So this case does not occur. If there is no node to the left of $L_{i}$, then $Y=B_{m}$ and we are in one of the cases studied in [4], which tells us there are no examples.

So we are left with the case when the embedding $L_{X}^{\prime} \hookrightarrow L_{i}$ is an isomorphism, so $L_{i}$ is of type $A_{m-1}$ and the labelling of the high weight of $V /\left[V, Q_{Y}\right]$ on $\Pi\left(L_{i}\right)$ 
is the same as the labelling on $\Pi\left(L_{X}^{\prime}\right)$. The two possible pictures are
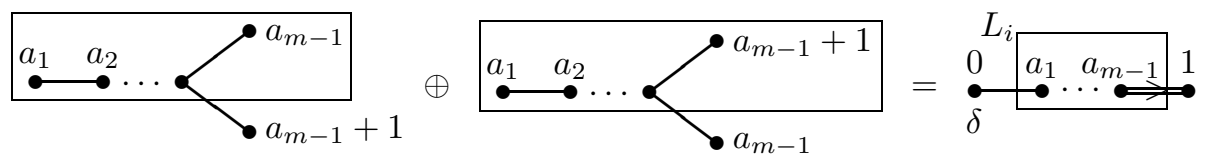

with the first summand projecting non-trivially to $V^{1}\left(Q_{Y}\right)$ and

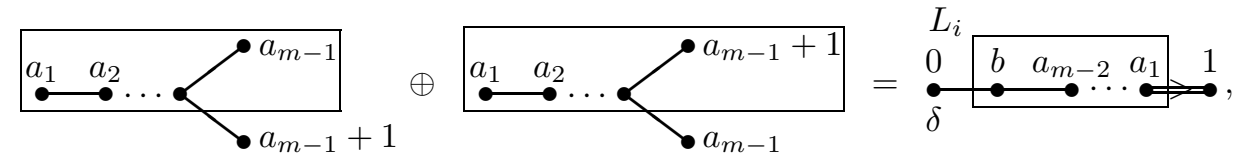

with $b=a_{m-1}$ if the first summand projects non-trivially to $V /\left[V, Q_{Y}\right]$ and $b=$ $a_{m-1}+1$ if it is the second summand which projects non-trivially (as noted at the beginning of this proof, all labels to the left of the highlighted nodes of the Dynkin diagram for $Y$ in the pictures above are 0 ).

If we have picture (1) above, then the question is whether or not $\delta$ exists. The $B_{m}$-module with high weight $a_{1} \lambda_{1}+\cdots+a_{m-1} \lambda_{m-1}+\lambda_{m}$ (where the $\lambda_{i}$ are for the moment the fundamental dominant weights of this Levi factor of $Y$ of type $B_{m}$ ), when considered as a module for the $D_{m}$ sitting in $B_{m}$ in the usual way, has high weights $a_{1} \delta_{1}+\cdots+a_{m-1} \delta_{m-1}+\left(a_{m-1}+1\right) \delta_{m}$ and $a_{1} \delta_{1}+\cdots+\left(a_{m-1}+1\right) \delta_{m-1}+$ $a_{m-1} \delta_{n}$ (this was noted in the proof of $[4,3.3]$ ). So if $\delta$ exists, then $V$ is too large to be the sum of two $D_{m}$-modules of these high weights. So $Y$ in fact has type $B_{m}$; but then we are back in the situation of [4], which tells us the only possibility here is the one in the statement of the theorem.

If we have picture (2) above, then the relationship between the $a_{i}$ which we know from [4] tells us that $a_{2}=a_{m-2}, a_{3}=a_{m-3}, \ldots$, and therefore $a_{1}=a_{m-1}$. If the first summand projects non-trivially to $V^{1}\left(Q_{Y}\right)$, then we in fact have an instance of picture (2), which we covered above.

So we may assume we have picture (2), with the second summand projecting non-trivially, i.e. the isomorphism $L_{X}^{\prime} \rightarrow L_{i}$ is given by the graph isomorphism sending $\beta_{1} \rightarrow \alpha_{m-1}, \beta_{2} \rightarrow \alpha_{m-2}, \ldots, \beta_{m-1} \rightarrow \alpha_{1}$ (here and below, the $\alpha_{i}$ are the fundamental roots of $Y$ corresponding to the "end" $m$ nodes of the Dynkin diagram, with the $\lambda_{i}$ the corresponding fundamental dominant weights).

We now define a normal subgroup of $P_{Y}$ which is the "one level down" analogue of $K_{Y}^{\gamma}$ (see Lemma 2.10 and the paragraph preceding it). For a root $\gamma \in \Pi(Y)-\Pi\left(L_{Y}\right)$, let $\Sigma_{Y}(\gamma)$ be the set of roots in $\Sigma^{-}(Y)$ which have $\gamma$-coefficient -1 and coefficient 0 for other fundamental roots not in $\Pi\left(L_{Y}\right)$. Then as in the introduction, $K_{Y}^{\gamma}$ is the product of root groups $U_{\beta}$ for $\beta \in\left(\Sigma^{-}(Y)-\Sigma^{-}\left(L_{Y}\right)-\Sigma_{Y}(\gamma)\right)$. Analogously, let $\Sigma_{Y}(2 \gamma)$ be the set of roots in $\Sigma^{-}(Y)$ having $\gamma$-coefficient -2 and coefficient 0 for other roots in $\Pi(Y)-\Pi\left(L_{Y}\right)$. Then let $K_{Y}^{2 \gamma}$ be the product of root groups $U_{\beta}$ for $\beta \in\left(\Sigma^{-}(Y)-\Sigma^{-}\left(L_{Y}\right)-\Sigma_{Y}(\gamma)-\Sigma_{Y}(2 \gamma)\right)$. $K_{Y}^{2 \gamma}$ is normal in $P_{Y}$ by the commutator relations, and $K_{Y}^{2 \gamma} \leq K_{Y}^{\gamma}$. We have previously considered the quotient $Q_{Y} / K_{Y}^{\gamma}$; now we wish to look at $K_{Y}^{\gamma} / K_{Y}^{2 \gamma}$. Let $\gamma=\alpha_{m}$ be the short fundamental root of $Y$. Then $K_{Y}^{\alpha_{m}} / K_{Y}^{2 \alpha_{m}} \cong$ the irreducible $L_{i}$-module with high weight $-\left.\left(2 \alpha_{m}+\alpha_{m-1}\right)\right|_{T_{L_{i}}}=\left.\lambda_{m-2}\right|_{T_{L_{i}}}$. 
Consider $Q^{\beta_{m}}=Q_{X} / K^{\beta_{m}}$, where $K^{\beta_{m}}$ is the product of those $T_{X}$-root groups $U_{\gamma}$ for $\gamma \in \Sigma^{-}(X)$ with $\beta_{m}$-coefficient less than -1 (which in this case is trivial, as there are no such roots in a root system of type $D_{m}$ ). By Lemma $2.10, Q^{\beta_{m}}$ is an $L_{X}^{\prime}$-module with high weight $\left.\left(-\beta_{m}\right)\right|_{T_{L_{X}}^{\prime}}=\left.\delta_{m-2}\right|_{T_{L_{X}}^{\prime}}$ and thus dimension $\left(\begin{array}{c}m \\ 2\end{array}\right)$.

By $[9,3.1], Q_{Y} / K_{Y}^{\alpha_{m}}$ is an irreducible $L_{Y}^{\prime}$-module with high weight $-\left.\alpha_{m}\right|_{T_{L_{Y}^{\prime}}}=$ $\left.\lambda_{m-1}\right|_{T_{L_{Y}^{\prime}}}$, which implies that $Q_{Y} / K_{Y}^{\alpha_{m}}$ has dimension $m$. It has an $L_{X}$-submodule $Q_{X} K_{Y}^{\alpha_{m}} / K_{Y}^{\alpha_{m}}$ (by $[9,3.3]$ ). If $Q_{X} \not \leq K_{Y}^{\alpha_{m}}$, then we see that $Q_{X} K_{Y}^{\alpha_{m}} / K_{Y}^{\alpha_{m}} \cong$ $Q_{X} /\left(Q_{X} \cap K_{Y}^{\alpha_{m}}\right) \cong Q_{X} / Q_{X}^{\prime} \cong Q_{X} / K^{\beta_{m}}$ has dimension $\left(\begin{array}{c}m \\ 2\end{array}\right)>m$, which is impossible. So $Q_{X} \leq K_{Y}^{\alpha_{m}}$. Then $K^{\beta_{m}}=Q_{X}^{\prime} \leq K_{Y}^{\alpha_{m}{ }^{\prime}} \leq K_{Y}^{2 \alpha_{m}}$, and we can project $Q_{X} / K^{\beta_{m}} \longrightarrow K_{Y}^{\alpha_{m}} / K_{Y}^{2 \alpha_{m}}$. If this map has non-zero image then it is in fact an isomorphism, since $Q_{X} / K^{\beta_{m}}$ is an irreducible $L_{X}^{\prime}$-module and $\operatorname{dim}\left(Q_{X} / K^{\beta_{m}}\right)=\operatorname{dim}\left(K_{Y}^{\alpha_{m}} / K_{Y}^{2 \alpha_{m}}\right)$. But then $K_{Y}^{\alpha_{m}} / K_{Y}^{2 \alpha_{m}}$ has $L_{X}^{\prime}$-high weight $\delta_{m-2}$, whereas we know, by the fact that the isomorphism $L_{X}^{\prime} \rightarrow L_{i}$ is given by the graph isomorphism sending $\beta_{1} \rightarrow \alpha_{m-1}, \beta_{2} \rightarrow \alpha_{m-2}, \ldots, \beta_{m-1} \rightarrow \alpha_{1}$, that $K_{Y}^{\alpha_{m}} / K_{Y}^{2 \alpha_{m}}$ must have $L_{X}^{\prime}$-high weight $\delta_{2}$ (since it has $L_{Y}^{\prime}$-high weight $\lambda_{m-2}$ ). So $2=m-2$, i.e. $X$ is of type $D_{4}$.

On the other hand, if $Q_{X} \leq K_{Y}^{2 \alpha_{m}}$ (i.e. if the map above is not an isomorphism), then we note that the roots $\beta$ whose corresponding root subgroups appear in $K_{Y}^{2 \alpha_{m}}$ all involve some fundamental root in $\Pi(Y)-\Pi\left(L_{Y}\right)$ other than $\alpha_{m}$ (since $3 \alpha_{m}$ appears in no root). So if we let $P_{Y}^{1}=\left\langle P_{Y}, U_{ \pm \alpha_{m}}\right\rangle$, we have a new parabolic subgroup of $Y$ satisfying $Q_{Y}^{1} \geq Q_{X}, L_{Y}^{1} \geq L_{X}, P_{Y}^{1} \geq P_{X}$. If now $V /\left[V, Q_{Y}^{1}\right]$ is irreducible as an $L_{X}^{\prime}$-module, then the first part of the proof of this lemma gives a contradiction. If $V /\left[V, Q_{Y}^{1}\right]$ is the sum of two irreducibles for $L_{X}^{\prime} \cong L_{i}$, then we have a contradiction because this case would have to appear in [4], and it does not.

So we are left with the picture
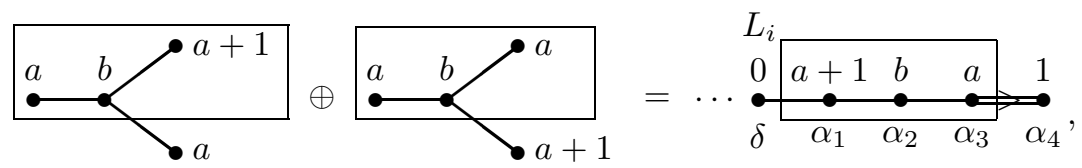

with the first summand projecting non-trivially to $V^{1}\left(Q_{Y}\right)$. In fact there are three possibilities: $a=(p-3) / 2, b=(p+1) / 2 ; a=0, b=(p-5) / 2$; or $a=b=0$. If $a=b=0$, then the $T_{Y}$-high weight of $V$ has at least 32 conjugates, making $V$ too large to be the sum of two $D_{4}$-modules of dimension 8 . We may assume $\operatorname{rank}(Y)=n \geq 5$, since otherwise [4] tells us there are no examples.

Suppose $a=0, b=(p-5) / 2$. Then $V_{1}$ has $D_{4}$-high weight $\frac{p-5}{2} \delta_{2}+\delta_{4}$, and $V$ has dimension at least the dimension of the $B_{5}$-module with high weight $\lambda_{1}+\frac{p-5}{2} \lambda_{2}+\lambda_{4}$ (the $\lambda_{i}$ refer to the fundamental weights corresponding to the end four nodes of the Dynkin diagram for $Y$ ). Thus $V$, as a module for the obvious $B_{4}<Y$, has a composition factor $V^{\prime}$ of high weight $\lambda_{1}+\frac{p-5}{2} \lambda_{2}+\lambda_{4}$ and another, $V^{\prime \prime}$, of high weight $\frac{p-3}{2} \lambda_{2}+\lambda_{4}$ (if $P_{Y}$ is the parabolic subgroup of $Y$ corresponding to the end four nodes, then $V^{2}\left(Q_{Y}\right)$ has an $L_{Y}$-high weight $\lambda-\delta-\alpha_{1}$, with the above restriction to $\left.T_{L_{Y}^{\prime}}\right)$. As a module for the $D_{4}<B_{4}, V^{\prime}$ has high weights $\delta_{1}+\frac{p-5}{2} \delta_{2}+\delta_{4}$ and $\delta_{1}+\frac{p-5}{2} \delta_{2}+\delta_{3}$, while $V^{\prime \prime}$ has high weights $\frac{p-3}{2} \delta_{2}+\delta_{4}$ and $\frac{p-3}{2} \delta_{2}+\delta_{3}$. So $\operatorname{dim}(V) \geq$ $2 \operatorname{dim}\left(V_{D_{4}}\left(\delta_{1}+\frac{p-5}{2} \delta_{2}+\delta_{4}\right)\right)+2 \operatorname{dim}\left(V_{D_{4}}\left(\frac{p-3}{2} \delta_{2}+\delta_{4}\right)\right)$. Using the Andersen-Jantzen 
sum formula $([1,6])$, we see that

$$
\begin{aligned}
\operatorname{dim}\left(V_{D_{4}}\left(\delta_{1}+\frac{p-5}{2} \delta_{2}+\delta_{4}\right)\right)= & \operatorname{dim}\left(W_{D_{4}}\left(\delta_{1}+\frac{p-5}{2} \delta_{2}+\delta_{4}\right)\right) \\
& -\operatorname{dim}\left(W_{D_{4}}\left(\delta_{1}+\frac{p-9}{2} \delta_{2}+\delta_{4}\right)\right)
\end{aligned}
$$

and

$$
\begin{aligned}
\operatorname{dim}\left(V_{D_{4}}\left(\frac{p-3}{2} \delta_{2}+\delta_{4}\right)\right)= & \operatorname{dim}\left(W_{D_{4}}\left(\frac{p-3}{2} \delta_{2}+\delta_{4}\right)\right) \\
& -\operatorname{dim}\left(W_{D_{4}}\left(\frac{p-9}{2} \delta_{2}+\delta_{4}\right)\right),
\end{aligned}
$$

where $W_{D_{4}}(\beta)$ denotes the Weyl module for $D_{4}$ with high weight $\beta$ (the second terms on the right hand sides of the above equalities appear only if $p-9 \geq 0$ ). Calculating these dimensions with the Weyl character formula, we find that

$$
\begin{aligned}
\operatorname{dim}(V) & \geq 2\left(\operatorname{dim}\left(V_{D_{4}}\left(\delta_{1}+\frac{p-5}{2} \delta_{2}+\delta_{4}\right)\right)+\operatorname{dim}\left(V_{D_{4}}\left(\frac{p-3}{2} \delta_{2}+\delta_{4}\right)\right)\right) \\
& >2 \operatorname{dim}\left(W_{D_{4}}\left(\frac{p-5}{2} \delta_{2}+\delta_{4}\right)\right) \\
& \geq 2 \operatorname{dim}\left(V_{D_{4}}\left(\frac{p-5}{2} \delta_{2}+\delta_{4}\right)\right) \\
& =2 \operatorname{dim}\left(V_{1}\right)=\operatorname{dim}\left(V_{1}\right)+\operatorname{dim}\left(V_{2}\right) .
\end{aligned}
$$

This is a contradiction, so in fact this case does not occur.

Finally, if $a \neq 0 \neq b$, then we take yet another parabolic: Let $P_{X}$ be the parabolic subgroup of $X$ corresponding to $\left\{\beta_{1}, \beta_{3}, \beta_{4}\right\} \subseteq \Pi(X)$. Then $L_{X}^{\prime}$ is a product of three $A_{1}$ 's. Here $P_{X}$ is $t$-stable, so when we embed $P_{X}$ in a parabolic subgroup $P_{Y}$ of $Y$, as usual, we have $\left[V, Q_{Y}\right]=\left[V, Q_{X}\right]$. We denote $T_{L_{X}^{\prime}}$-weights by $\left(a_{1}, a_{3}, a_{4}\right)$, where $a_{i} \in \mathbf{Z}$ is the value of the weight on the torus corresponding to $\beta_{i}$. The high weight of $V_{1} /\left[V_{1}, Q_{X}\right]$ is $(a, a+1, a)$; that of $V_{2} /\left[V_{2}, Q_{X}\right],(a, a, a+1)$. The weights which appear in $V_{1} /\left[V_{1}, Q_{X}\right]$ have the form $\left(a-2 b_{1}, a+1-2 b_{3}, a-2 b_{4}\right)$, and those in $V_{2} /\left[V_{2}, Q_{X}\right]$ have the form $\left(a-2 c_{1}, a-2 c_{3}, a+1-2 c_{4}\right)$. The weight spaces in $V_{i} /\left[V_{i}, Q_{X}\right]$ all have dimension 1 , and no weight can appear in both. So the weight spaces of $V /\left[V, Q_{Y}\right]$ have dimension 1 .

Those modules for simple algebraic groups which have all weight spaces of dimension 1 are classified in $[9$, chapter 6$]$. We have $\operatorname{dim}\left(V_{1} /\left[V_{1}, Q_{X}\right]\right)=(a+1)^{2}(a+2)$, so $\operatorname{dim}\left(V /\left[V, Q_{Y}\right]\right)=2(a+1)^{2}(a+2)$. With the labelling on $Y$ known, we can compare the various possibilities for the factors of $L_{Y}^{\prime}$ which act non-trivially on $V^{1}\left(Q_{Y}\right)$ (remembering that they must appear in $[9,6.1]$ ) with the known dimension of $V^{1}\left(Q_{Y}\right)$, and we get a contradiction in every case but one: $p=13$, with the high weights and the embedding of $P_{X}$ in $P_{Y}$ as in the picture

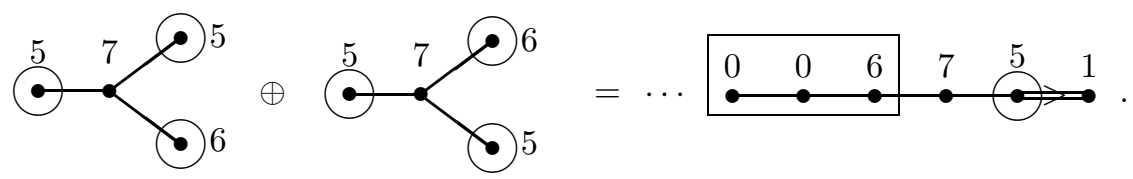

But now the $L_{Y}^{\prime}$-module $Q_{Y} / K_{Y}^{\gamma}$ has dimension 2, while $Q_{X} / Q_{X}^{\prime}$ has dimension 8; this forces $Q_{X} \leq K_{Y}^{\gamma}$ (otherwise $Q_{X} K_{Y}^{\gamma} / K_{Y}^{\gamma} \cong Q_{X} / Q_{X}^{\prime}$ is a submodule of dimension 8 of $Q_{Y} / K_{Y}^{\gamma}$, which is impossible). But then $V_{\lambda-\gamma} \notin\left[V, Q_{X}\right]=\left[V, Q_{Y}\right]$, which is absurd since $\gamma$ has a non-zero label. 
So we get no examples here.

4.4. Case 4. We are left with case 4 on as stated above Lemma 4.2.

Lemma 4.6. If $W_{i}$ is irreducible for $L_{X}^{\prime}$, then $p=2, X=D_{n}$, and $Y=B_{n}$ or $C_{n}$, with $V$ a spin module for $Y$ and a sum of two spin modules for $X$.

Proof. We are back to the situation where $P_{X}$ is the maximal parabolic subgroup corresponding to $\Pi(X)-\left\{\beta_{1}\right\}$. Inductively we need only to check the case where $p=2, L_{X}^{\prime}=D_{m-1}$, and $L_{i}=B_{m-1}$ or $C_{m-1}$, with $V /\left[V, Q_{Y}\right]$ a spin module for $L_{i}$ and a sum of two spin modules for $L_{X}^{\prime}$; and the single case which occurred in section 3: $L_{i}=D_{10},\left.\left(V /\left[V, Q_{Y}\right]\right)\right|_{L_{i}}=\operatorname{spin}\left(L_{i}\right), L_{X}^{\prime}=D_{3}=A_{3}, V_{1} /\left[V_{1}, Q_{X}\right]$ of $L_{X}$-high weight $3 \delta_{3}+\delta_{2}+\delta_{1}, p \neq 2,3,5,7$.

If we are in the first setup, then the arguments of the last subsection carry over and we have only the examples in the statement of the Lemma.

So the picture is

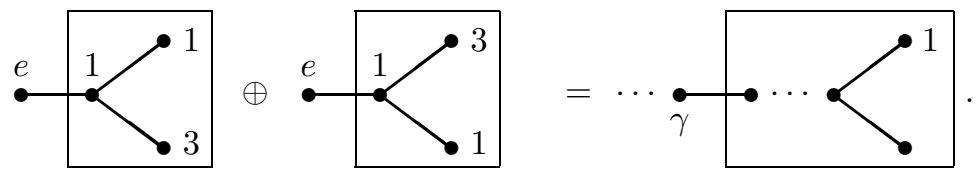

Assume $\gamma$ has a non-zero label (in the marking for the $Y$-high weight of $V$ ). Then $V_{\gamma}\left(Q_{Y}\right)$ has an $L_{Y}^{\prime}$-high weight given by the labelling on the boxed nodes above with a 1 on the node to the right of $\gamma$ and the 1 on the end node as pictured. The dimension of this $D_{10}$-module is at least the number of conjugates of the high weight, which is $2^{9} \cdot 10$. But then $\operatorname{dim}\left(V^{2}\left(Q_{Y}\right)\right) \geq \operatorname{dim}\left(V_{\gamma}\left(Q_{Y}\right)\right) \geq 2^{9} \cdot 10>6 \cdot 2^{9}=$ $6 \operatorname{dim}\left(V^{1}\left(Q_{Y}\right)\right)=\operatorname{dim}\left(Q^{\alpha}\right) \operatorname{dim}\left(V^{1}\left(Q_{Y}\right)\right)$, which is a contradiction to Lemma 2.9. So $\gamma$ has label 0. By Lemma 4.3, all the other nodes in the diagram for $Y$ have label 0 , so in fact $V$ is a spin representation of $Y$.

Now switch parabolics: Let $P_{X}$ correspond to $\left\{\beta_{1}, \beta_{2}\right\}<\Pi(X)$. As always, embed $P_{X}$ in a parabolic $P_{Y}$ of $Y$, via the usual construction (given in Lemma 2.7). Since $P_{X}$ is $t$-stable, we again have in this case $\left[V, Q_{Y}\right]=\left[V, Q_{X}\right]$ by Lemma 2.8 . Since $V /\left[V, Q_{X}\right] \neq 0$, the subset of $\Pi(Y)$ to which $P_{Y}$ corresponds must contain the node $\alpha$ that has a label 1 . Let $L_{i}$ be the simple factor of $L_{Y}$ that contains $\left\langle U_{\alpha}\right\rangle$.

The irreducible $L_{X}^{\prime}$-module $V_{1} /\left[V_{1}, Q_{X}\right]$ has high weight $\left.\left(e \delta_{1}+\delta_{2}\right)\right|_{T_{L_{X}}^{\prime}}$. Its dimension is $(e+1)(e+3)$ if $e \neq p-2$, and $\frac{(e+1)(e+6)}{2}$ if $e=p-2$. Recall that $\operatorname{dim}\left(V /\left[V, Q_{Y}\right]\right)=2\left(\operatorname{dim}\left(V_{1} /\left[V_{1}, Q_{X}\right]\right)\right)$.

If $L_{i}$ has type $D_{l}$, then $\operatorname{dim}\left(V_{1} /\left[V_{1}, Q_{X}\right]\right)$ is a power of 2 . The dimension $(e+1)(e+3)$ is a power of 2 only when $e=1$, and $\frac{(e+1)(e+6)}{2}$ is never a power of 2. So the possibility here is $e=1, p \neq 3$.

If $L_{i}$ has type $A_{l}$ and $e>0$, then $l \geq \frac{(e+1)(e+6)}{2}-1$ (which is always $\geq 6$, so $\Pi\left(L_{i}\right)$ does not contain both $\alpha_{n}$ and $\left.\alpha_{n-1}\right)$. Then $V^{2}\left(Q_{Y}\right)$ has a composition factor of high weight $\left.\left(\lambda-\gamma_{l}-\gamma_{l-1}-\alpha\right)\right|_{T_{L_{Y}}}$ (where $\gamma_{l-1}, \gamma_{l}$ are the end nodes of $L_{i}$ and $\alpha$ is the node at the end of $Y$ which is left out of $\left.L_{i}\right)$, of dimension $\left(\begin{array}{c}l+1 \\ 3\end{array}\right)$. So we have $\left(\begin{array}{c}l+1 \\ 3\end{array}\right) \leq 6 \operatorname{dim}\left(V^{1}\left(Q_{Y}\right)\right)=6(l+1)$ (using Lemma 2.9 again). But $\left(\begin{array}{c}l+1 \\ 3\end{array}\right) \leq 6(l+1)$ is a contradiction for $l \geq 6$, and $l \leq 5$ only for $e=0$.

We are left with some cases for $e=1$ and $e=0$. But $V$ is a spin module for $Y$, so $\operatorname{dim}(V)$ is a power of 2 ; the only time when $V_{1}$ as above has dimension a power of 2 is for $e=1$ and $p>11$ or $p=0$, in which case $\operatorname{dim}\left(V_{1}\right)=2^{15}$. But this would 
imply that $\operatorname{dim}(V)=2^{16}$, so $Y$ has type $D_{17}$. But $X=D_{4}$ has no irreducible representations of dimension $34(=\operatorname{dim}(W))$ when $p>11$ or $p=0$.

This completes the proof of the theorem.

\section{The CASE $X=E_{6}$}

Here we establish Theorem 1 for the case where $X=E_{6}$ and $G=X\langle t\rangle$. Again we assume that $t$ acts on $W$, the natural module for $Y$. Notation $\left(X \leq Y, V, V_{1}\right.$, $\left.V_{2}, \alpha_{i}, \lambda\right)$ is as previously defined; in particular, $\left\{\beta_{1}, \ldots, \beta_{6}\right\}$ is the set of simple roots of $X$, with $\left\{\delta_{1}, \ldots, \delta_{6}\right\}$ the set of fundamental dominant weights, labelled so that $\left\langle\delta_{i}, \beta_{j}\right\rangle=\delta_{i j}$. The main theorem is

Theorem 5.1. If $X$ acts irreducibly on the natural module $W$ for $Y$, then $X$ is not of type $E_{6}$.

Proof. Since $t$ acts on $W$, the $T_{X}$-high weight $\delta=d_{1} \delta_{1}+\cdots+d_{6} \delta_{6}$ of $W$ must be symmetric; that is, $d_{1}=d_{6}$ and $d_{3}=d_{5}$. We will write $\delta=a \delta_{1}+d \delta_{2}+b \delta_{3}+c \delta_{4}+$

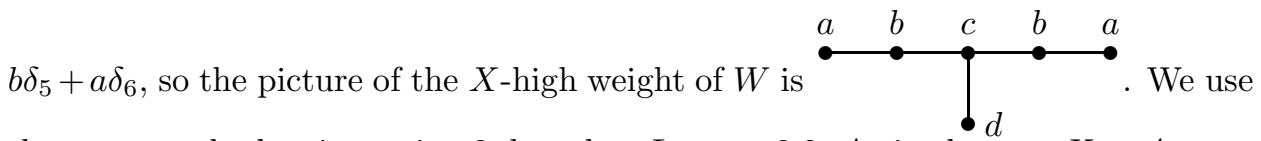

the same method as in section 3, based on Lemma 3.2. As in the case $X=A_{m}$, we will investigate the embedding of the fixed Borel subgroup $B_{X}$ of $X$ in a parabolic subgroup $P_{Y}$ of $Y$, via the construction outlined in the proof of Lemma 2.7. In the first subsection, we will show that there are only a few cases in which a factor of type $A_{1}$ might appear in $L_{Y}^{\prime}$; following that, we deal with these few cases by investigating the embeddings of other parabolic subgroups of $X$.

5.1. The Almost-Everywhere Argument. The argument will again be an induction on the partial order on the weight lattice; for the base case of the induction we need

Lemma 5.2. If $X$ is of type $E_{6}$ and $\delta \neq \delta_{2}$, then $\delta \succ \delta_{1}+\delta_{6}$.

Proof. This is an easy exercise (using the fact that $\delta$ is symmetric).

Notice that 0 is a weight of $V_{E_{6}}\left(\delta_{1}+\delta_{6}\right)$ at level 16 by the expression for $\delta_{1}+\delta_{6}$ in terms of roots (see [5], for example). So to begin our induction we must show that in $V_{E_{6}}\left(\delta_{1}+\delta_{6}\right)=V_{E_{6}}(\delta)$ there are at least three weights in every $U_{X}$-level $i$ for $2 \leq i<16$ and at least five at level 16. This is easy to do; we illustrate by giving three weights in each of levels $2-4$. Here we are using the usual rules to determine that a weight appears: If $\mu$ is a $T_{X}$-weight such that $W_{\mu} \neq 0$, and $\left\langle\mu, \beta_{i}\right\rangle=l>0$, then $W_{\mu-j \beta_{i}} \neq 0$ for every $1 \leq j \leq l$. This depends on the result in [8], which says that weights which appear in characteristic 0 also appear in characteristic $p$, and the fact that in characteristic 0 , the $\beta_{i}$-string through $\mu$ is connected (the set of weights is saturated - see [5, p. 114]).

Level 2: $\delta-\beta_{1}-\beta_{3}, \delta-\beta_{6}-\beta_{5}, \delta-\beta_{1}-\beta_{6}$;

Level 3: $\delta-\beta_{1}-\beta_{3}-\beta_{4}, \delta-\beta_{6}-\beta_{5}-\beta_{4}, \delta-\beta_{1}-\beta_{3}-\beta_{6}$;

Level 4: $\delta-\beta_{1}-\beta_{3}-\beta_{4}-\beta_{5}, \delta-\beta_{6}-\beta_{5}-\beta_{4}-\beta_{3}, \delta-\beta_{1}-\beta_{3}-\beta_{4}-\beta_{6}$;

etc. 
If $\delta=\delta_{2}$, then we check all levels and find that the only possibilities for an $A_{1}$-factor of $L_{Y}^{\prime}$ are level 3 and level 11 (the level of the 0 weight). The weight space for the weight 0 has dimension at least 5 in all characteristics, so level 11 is large enough to preclude a corresponding $a_{1}$-factor of $L_{Y}^{\prime}$. We will deal with level 3 later.

Since $\delta$ is symmetric, it has the form $a\left(\delta_{1}+\delta_{6}\right)+b\left(\delta_{3}+\delta_{5}\right)+c \delta_{4}+d \delta_{2}$ for some nonnegative integers $a, b, c, d$. Now for the induction. Assume $\delta \succ \delta_{1}+\delta_{6}$ (and $\delta \neq \delta_{1}+\delta_{6}$ ). Then at least one of the following must be true: 1) $d \geq 2$;2) $c \geq 2$; 3) $b>0$; 4) $a>0$; or 5) $\delta \in\left\{\delta_{4}, \delta_{2}+\delta_{4}\right\}$. We consider each of these possibilities in turn.

1) If $d \geq 2$, then $\delta-\beta_{2}$ is a dominant weight, still greater than $\delta_{1}+\delta_{6}$ in the partial order, and by induction $\delta$ has enough weights at all levels 3 and higher. So we need to check levels 1 and 2. Level 1 is 2-dimensional if $a=b=0, c \neq 0$; otherwise $\operatorname{dim}\left(W_{1}\right)=1$ or $\operatorname{dim}\left(W_{1}\right) \geq 3$. At level 2 , if $a=b=c=0$, we have just the two weights $\delta-2 \beta_{2}, \delta-\beta_{2}-\beta_{4}$; so this is a case we must consider below.

2) If $c>1$, then $\delta-\beta_{4}$ is a dominant weight, still greater than $\delta_{1}+\delta_{6}$ in the partial order, and by induction $\delta$ has enough weights at all levels 3 and higher. So we need to check levels 1 and 2. As above, level 2 is 2-dimensional if $a=b=0, d \neq 0$. At level 2 are the three weights $\delta-\beta_{4}-\beta_{3}, \delta-\beta_{4}-\beta_{2}$, and $\delta-\beta_{4}-\beta_{5}$.

3) If $b>0$, then $\delta-\beta_{3}-\beta_{4}-\beta_{5}$ is a dominant weight, still greater than $\delta_{1}+\delta_{6}$, and by induction we must check levels $1-4$. Level 1 is 2-dimensional only if $a=c=d=0$; level 2 has at least 5 weights in it; level 3, at least 5 ; and level 4 , at least 5 . So for $b \neq 0$ we need to consider only level 1 with $a=c=d=0$.

4) If $a>0$ and $\delta \neq \delta_{1}+\delta_{6}$, then $\delta-\beta_{1}-\beta_{3}-\beta_{4}-\beta_{5}-\beta_{6}$ is a dominant weight, and we need to check levels $1-6$. Level one is 2-dimensional only if $b=c=d=0$; level 2 has at least 5 weights appearing; level 3 , at least 4 ; level 4 , at least 5 ; level 5 , at least 5 ; and level 6 , at least 5 . So for $a \neq 0$, we have only to consider level 1 with $b=c=d=0$.

5) The weights that aren't covered above are $\delta=\delta_{4}, \delta=\delta_{2}+\delta_{4}$, and $\delta=\delta_{2}$. Notice that $\delta_{4}-\beta_{3}-\beta_{2}-2 \beta_{4}-\beta_{5}=\delta_{1}+\delta_{6}$, so we need to check levels 1-6; in this case, level 1 has dimension 1 and levels $2-6$ all have dimension at least 3 . So $\delta=\delta_{4}$ gives no $A_{1}$-factors of $L_{Y}^{\prime}$. If $\delta=\delta_{2}+\delta_{4}$, then $\delta-\beta_{4}-\beta_{2}$ is a dominant weight, so we need to check only levels $1-3$. Level 1 has dimension 2 , so it must be considered; levels 2 and 3 are both big enough.

So the only embeddings for which an $A_{1}$ factor of $L_{Y}^{\prime}$ might appear are the ones that give an obvious level of dimension 2 :

1. Level 1 for $\delta=a \delta_{1}+a \delta_{6} ; \delta=b \delta_{3}+b \delta_{5} ;$ or $\delta=c \delta_{4}+d \delta_{2}(a \neq 0 \neq b, c \neq 0 \neq d)$.

2. Level 2 for $\delta=d \delta_{2}$ with $d>1$.

3. Level 3 for $\delta=\delta_{2}$.

5.2. The Remaining Cases. We treat the cases listed above in reverse order.

3. Say $\delta=\delta_{2}$. Then the picture of the embedding of the Borel subgroup of $X$ is

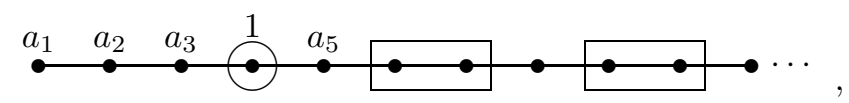

with all simple factors of $L_{Y}^{\prime}$ to the right of the picture having rank at least 2, all separated by only a single node.

By Lemma 2.4, we have $\operatorname{dim}\left(V^{2}\left(Q_{Y}\right)\right) \leq \operatorname{dim}\left(V^{2}\left(Q_{X}\right)\right)=2 \operatorname{dim}\left(V_{1}^{2}\left(Q_{X}\right)\right) \leq$ $2 \cdot 6 \operatorname{dim}\left(V_{1}^{1}\left(Q_{X}\right)\right)=12$. No node other than $\alpha_{4}$ of the Dynkin diagram for $Y$ 
which falls in $\Pi\left(L_{Y}^{\prime}\right)$ can have a nonzero label in the labelling for $\lambda$ on $Y$, since $\operatorname{dim}\left(V^{1}\left(Q_{Y}\right)\right)=2$. Suppose some node $\alpha_{i} \in \Pi(Y)-\Pi\left(L_{Y}\right)^{\prime}$ for $i>7$ has a nonzero label. Then $\left.\left(\lambda-\alpha_{i}\right)\right|_{T_{L_{Y}^{\prime}}}$ is a high weight in $V^{2}\left(Q_{Y}\right)$, giving a composition factor of dimension at least 9 (since the two simple factors of $L_{Y}^{\prime}$ which it adjoins have rank $\geq 2$ ). But $\lambda-\alpha_{4}-\alpha_{3}$ (if $a_{3}=0$ ) or $\lambda-\alpha_{3}$ (if $a_{3}>0$ ) is another high weight in $V^{2}\left(Q_{Y}\right)$, giving a factor of dimension at least 1 , and $\lambda-\alpha_{4}-\alpha_{5}$ (if $a_{5}=0$ ) or $\lambda-\alpha_{5}$ (if $a_{5}>0$ ) is another, giving one of dimension at least 3. Adding these up, we see that $\operatorname{dim}\left(V^{2}\left(Q_{Y}\right)\right) \geq 13$, which is a contradiction.

So $a_{i}=0$ for $i>5$. Now embed the maximal parabolic subgroup $P_{X}$ of $X$ corresponding to $\beta_{2}$ into a parabolic subgroup $P_{Y}$ of $Y$ by the same $Q_{X}$-level construction. Here level 0 has dimension 1 and level 1 has dimension 20. Now $L_{X}^{\prime}$ has type $A_{5}$ and the factor $L_{1}$ of $L_{Y}^{\prime}$, corresponding to level 1, has type $A_{19}$. Since $a_{i}=0$ for $i>5, L_{1}$ is the only factor of $L_{Y}^{\prime}$ acting nontrivially on $V /\left[V, Q_{Y}\right]$. By Lemma 2.8, $V /\left[V, Q_{Y}\right]=V_{1} /\left[V_{1}, Q_{X}\right] \oplus V_{2} /\left[V_{2}, Q_{X}\right]$. But then we are in the situation of section 3 , with $V /\left[V, Q_{Y}\right]$ an irreducible $A_{5}\langle t\rangle$-module. Since there were no examples there of this setup, inductively we have none here.

2. If $\delta=d \delta_{2}$ with $d>1$, then the embedding of the Borel subgroup of $X$ in a parabolic subgroup of $Y$ is

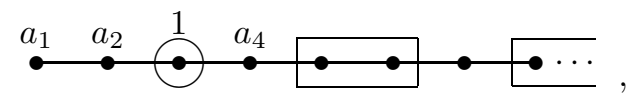

with, as above, all simple factors of $L_{Y}^{\prime}$ to the right of the picture of rank at least 2 , all separated by only a single node. We continue exactly as in case 3 , finding no examples.

1. We have $\delta=a \delta_{1}+a \delta_{6}, \delta=b \delta_{3}+b \delta_{5}$, or $\delta=c \delta_{4}+d \delta_{2}$. The embedding of $B_{X}$ in a parabolic subgroup of $Y$ is as pictured in

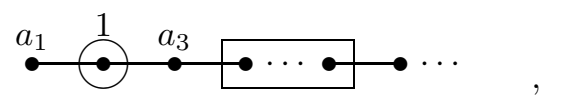

with the second simple factor of $L_{Y}^{\prime}$ having type $A_{l}$ for $l \geq 2$ and all simple factors of $L_{Y}^{\prime}$ to the right of the picture having rank at least 2, all separated by only a single node. By the same arguments as above, $a_{i}=0$ for $i>4$.

We again embed the maximal parabolic subgroup of $X$ corresponding to $\beta_{2}$ in a parabolic subgroup of $Y$; now level 0 is large, with dimension at least 20. Because $a_{i}=0$ for $i>4$, the factor $L_{1}$ of $L_{Y}^{\prime}$ corresponding to this level 0 is the only simple factor of $L_{Y}^{\prime}$ acting nontrivially on $V /\left[V, Q_{Y}\right]$. As above, we are back in the situation of section 3 , which tells us that once again there are no examples.

So $G=E_{6}\langle t\rangle$, with $E_{6}$ acting irreducibly on $W$, gives no examples.

$$
\text { 6. The CASE } X=D_{4},[G: X] \geq 3
$$

In this section we treat the cases $G=D_{4}\langle s\rangle$ and $G=D_{4}\langle s, t\rangle$, where $s$ is a graph automorphism of $D_{4}=X$ of order 3 and $t$ is one of order 2. As mentioned earlier, since $G<\operatorname{Aut}(Y)$ we have $s \in Y$, as no simple algebraic group properly containing $D_{4}$ has an outer automorphism of order 3 . We assume that $s$ acts on $W$ and that $W$ is irreducible as an $X$-module. The case $G=D_{4}\langle t\rangle$ was covered in section 4. All notation is as before. The main result is

Theorem 6.1. If $X$ acts irreducibly on the natural module $W$ for $Y$, then we are not in the situation where $X=D_{4}$ with $G=X\langle s\rangle$ or $G=X\langle s, t\rangle$. 
Notice that the assumption that $s$ acts on $W$ forces the $T_{X}$-high weight $\delta$ of $W$ to be of the form $a \delta_{1}+b \delta_{2}+a \delta_{3}+a \delta_{4}$, which implies that $t$ acts on $W$. In addition, it implies that $X$ fixes a nondegenerate bilinear form on $W$, which is orthogonal if $p \neq 2([11$, Lemma 79]).

If $\left.V\right|_{X}=V_{1} \oplus V_{2} \oplus V_{3}$ with each $V_{i}$ irreducible and restricted as an $X=D_{4^{-}}$ module, then $s$ permutes the $V_{i}$ and $V$ is irreducible as an $X\langle s\rangle$-module; in this case we may assume that $G=X\langle s\rangle$. So if $G=X\langle s, t\rangle$, we may assume that $V$ has six simple factors as an $X$-module.

We based the arguments for $X=A_{m}$ and $X=E_{6}$ on Lemma 3.2. We will need an analogous result for the cases we consider here. Recall that $l_{\delta}$ is the $U_{X}$-level of the $T_{X}$-low weight of $W$. Let $P_{Y}$ be the parabolic subgroup of $Y$ containing $B_{X}=U_{X} T_{X}$, constructed via $U_{X}$-levels as outlined in Lemma 2.7, with $P_{Y}=Q_{Y} L_{Y}$ the Levi decomposition given in that construction.

Lemma 6.2. If $P_{Y}$ is as above, then $\operatorname{dim}\left(V /\left[V, Q_{Y}\right]\right)=3$ if $G=X\langle s\rangle$ and $\operatorname{dim}\left(V /\left[V, Q_{Y}\right]\right)=6$ if $G=X\langle s, t\rangle$. If $G=X\langle s\rangle$, either $\operatorname{dim}\left(W_{l_{\delta} / 2}\right)=4$ or there is a $U_{X}$-level in $W$ of dimension 3 or 2. If $G=X\langle s, t\rangle$, there is an $i$ such that $2 \leq \operatorname{dim}\left(W_{i}\right) \leq 6$.

Proof. It suffices to prove the statements in the first sentence, since $A_{1}, A_{2}$, and $A_{1} \times A_{1}$ are the only groups under consideration which have simple modules of dimension 3 , and only those groups whose natural modules have dimension at most 6 have an irreducible module of dimension 6 .

The construction via $U_{X}$-levels of the parabolic subgroup $P_{Y}$ of $Y$ clearly gives an $s$-stable subgroup, as $s$ acts on $W$ and on each $U_{X}$-level in $W$. The quotient $V /\left[V, Q_{Y}\right]$ is an irreducible $L_{Y}^{\prime}$-module by [9, Lemma 2.10]. By an argument similar to that used to prove Lemma 2.8, here we have $\left.\left(V /\left[V, Q_{Y}\right]\right)\right|_{L_{X}}=V_{1} /\left[V_{1}, U_{X}\right] \oplus$ $V_{2} /\left[V_{2}, U_{X}\right] \oplus V_{3} /\left[V_{3}, U_{X}\right]\left(\oplus V_{4} /\left[V_{4}, U_{X}\right] \oplus V_{5} /\left[V_{5}, U_{X}\right] \oplus V_{6} /\left[V_{6}, U_{X}\right]\right.$, if $\left.t \in G\right)$. But each of these $L_{X}$-modules $V_{i} /\left[V_{i}, U_{X}\right]$ has dimension 1 by Lemma 2.4. This proves the first statement of the Lemma.

Recall that $P_{Y}$ is the stabilizer in $Y$ of the flag $0 \leq W_{l} \leq W_{l} \oplus W_{l-1} \leq \cdots$, where $l$ is minimal with respect to $\left[W, U_{X}^{l+1}\right]=0$. Each factor $L_{i}$ of $L_{Y}^{\prime}$ corresponds to a $U_{X}$-level $W_{i}$. Since $L_{Y}^{\prime}$ has an irreducible module of dimension $j=3$ or 6 , there must be a simple factor of $L_{Y}^{\prime}$ of rank less than $j$. For $j=3$, this can happen if $i=l_{\delta} / 2$ and $\operatorname{dim}\left(W_{l_{\delta} / 2}\right)=4$ (we could have the middle level $l_{\delta} / 2$ giving a product of two groups of type $A_{1}$ if $\left.Y=D_{n}\right)$; otherwise, this happens only if $\operatorname{dim}\left(W_{i}\right) \leq j$. This proves the second assertion of the Lemma.

Now if we can show that for a particular $T_{X}$-high weight $\delta$ of $W$, all $U_{X}$-levels of $W$ have dimension bigger than $j$ (with $j$ as above), and in the $G=X\langle s\rangle$ case $\operatorname{dim}\left(W_{l_{\delta} / 2}\right)>4$, then the Lemma will imply that there are no examples with this embedding of $X$ into $Y$.

Lemma 6.3. Assume $X=D_{4}, s \in G$, and $X$ acts irreducibly on the natural module $W$ for $Y$. If $\operatorname{dim}\left(W_{i}\right) \leq 3$ where $W_{i}$ is the $U_{X}$-level $i$ of $W$, then one of the following holds:

1. $i=1$ and either $\delta=b \delta_{2}$ or $\delta=a \delta_{1}+a \delta_{3}+a \delta_{4}$ for some $b \neq 0 \neq a$.

2. $\delta=\delta_{2}, \delta=2 \delta_{2}$, or $\delta=\delta_{1}+\delta_{3}+\delta_{4}$.

If $\operatorname{dim}\left(W_{i}\right) \leq 6$, then one of the following holds:

3. $i=1$.

4. $i=2$ and either $\delta=b \delta_{2}$ for some $b \neq 0$ or $\delta=\delta_{1}+\delta_{2}+\delta_{3}+\delta_{4}$. 
5. $\delta=\delta_{2}, \delta=2 \delta_{2}$, or $\delta=\delta_{1}+\delta_{3}+\delta_{4}$.

Finally, if $\operatorname{dim}\left(W_{l_{\delta} / 2}\right) \leq 4$, then $\delta=\delta_{2}$.

Proof. We wish to induct on the height in the weight lattice of $\delta$, as in the $X=A_{m}$ and $X=E_{6}$ cases. Let

$$
\delta=a \delta_{1}+b \delta_{2}+a \delta_{3}+a \delta_{4}
$$

be the $T_{X}$-high weight of $W$. Since $\delta_{1}+\delta_{3}+\delta_{4}=2 \beta_{1}+3 \beta_{2}+2 \beta_{3}+2 \beta_{4}$ and $\delta_{2}=\beta_{1}+2 \beta_{2}+\beta_{3}+\beta_{4}$ are sums of roots, every weight which has the form of $\delta$ has $\delta_{1}+\delta_{2}+\delta_{3}+\delta_{4}$ as a subdominant weight, except $\delta_{2}, 2 \delta_{2}$, and $\delta_{1}+\delta_{3}+\delta_{4}$.

So to begin our induction we must investigate the numbers of weights at various $U_{X}$-levels of the $D_{4}$-module with high weight $\delta_{1}+\delta_{2}+\delta_{3}+\delta_{4}$. The weight 0 appears in this module at level 14, so we must check the numbers of weights at levels $i$ with $1 \leq i \leq 14$. It is not hard to do this (again using the result in [8] that weights which appear in characteristic 0 appear in characteristic $p$ ); we find that there are 4 weights at level 1, 6 at level 2 , and 10 or more at every level $3-14$. We will exclude level 1 from the discussion below, since it is clear that $\operatorname{dim}\left(W_{1}\right)=1$ if $a=0$ and $b>0 ; \operatorname{dim}\left(W_{1}\right)=3$ if $a>0$ and $b=0$; and $\operatorname{dim}\left(W_{1}\right)=4$ if $a \neq 0 \neq b$.

Assume $\delta=a \delta_{1}+b \delta_{2}+a \delta_{3}+a \delta_{4}$ as above, with $b>2$. Then $\delta-\beta_{2}$ is a dominant weight, less than $\delta$ in the partial order and still having $\delta_{1}+\delta_{2}+\delta_{3}+\delta_{4}$ as a subdominant weight, so by induction $\delta-\beta_{2}$ has enough weights at all levels 3 and higher. So we must check $\delta$-levels 2 and 3. There are 4 weights at level 2 if $a=0$, and 7 otherwise. There are at least 7 weights at level 3 . So the only possibility for a level of dimension 6 or less is level 2, with $a=0$.

If $b=2$ and $a>1$, then again $\delta-\beta_{2}$ is dominant and lower in the partial order, still with $\delta_{1}+\delta_{2}+\delta_{3}+\delta_{4}$ as a subdominant weight, so by induction $\delta-\beta_{2}$ has enough weights at all levels 3 and higher. Here, level 2 has 7 weights and level 3 has at least 7 . So we have no possibilities arising from this embedding (other than level 1).

Finally, if $b=1$ and $a>1$, then $\delta-\beta_{1}-\beta_{3}-\beta_{4}$ is dominant and lower in the partial order; so by induction we must check $\delta$-levels $2,3,4$, and 5 . At all 4 of these levels there are more than 7 weights. This completes the listing of all possibilities for levels of dimension at most 6 , except for the three weights $\delta_{2}, 2 \delta_{2}$, and $\delta_{1}+\delta_{2}+\delta_{3}$.

The last statement of the lemma is proved by noting that by the same argument as above, every symmetric dominant weight except $\delta_{1}$ has $\delta_{1}+\delta_{3}+\delta_{4}$ as a subdominant weight, and $\delta_{1}+\delta_{3}+\delta_{4}$ has more than 4 weights at level 9 , which is its middle level.

We see in the proof that the only possibilities for 1-dimensional $U_{X}$-levels are level 1 when $\delta=b \delta_{2}$ and level 0 . So as in the arguments for earlier cases, we have that for any $\delta$, there are never 2 consecutive weights in the Dynkin diagram for $Y$ which lie outside of $\Pi\left(L_{Y}^{\prime}\right)$, except for $\alpha_{1}$ and $\alpha_{2}$ in the case $\delta=b \delta_{2}$.

From this point, we will consider the two possibilities for $G$ separately.

6.1. $G=X\langle s\rangle$. Here we need to find a level of dimension 3 or 2. By the previous Lemma, we need to consider only level 1 for $\delta=a \delta_{1}+a \delta_{3}+a \delta_{4}$ and the three possible $\delta$ that were not covered by the induction there: $\delta_{2}, 2 \delta_{2}$, and $\delta_{1}+\delta_{3}+\delta_{4}$. We can check these last three directly and find that the only possibilities for a factor 
of $L_{Y}^{\prime}$ of type $A_{1}$ or $A_{2}$ are those corresponding to level 1 for $\delta=\delta_{1}+\delta_{3}+\delta_{4}$ and to levels 2,3 and 5 for $\delta=\delta_{2}$.

So we must consider the following:

$\left.W\right|_{X} \quad$ Embedding of $B_{X}$

1)
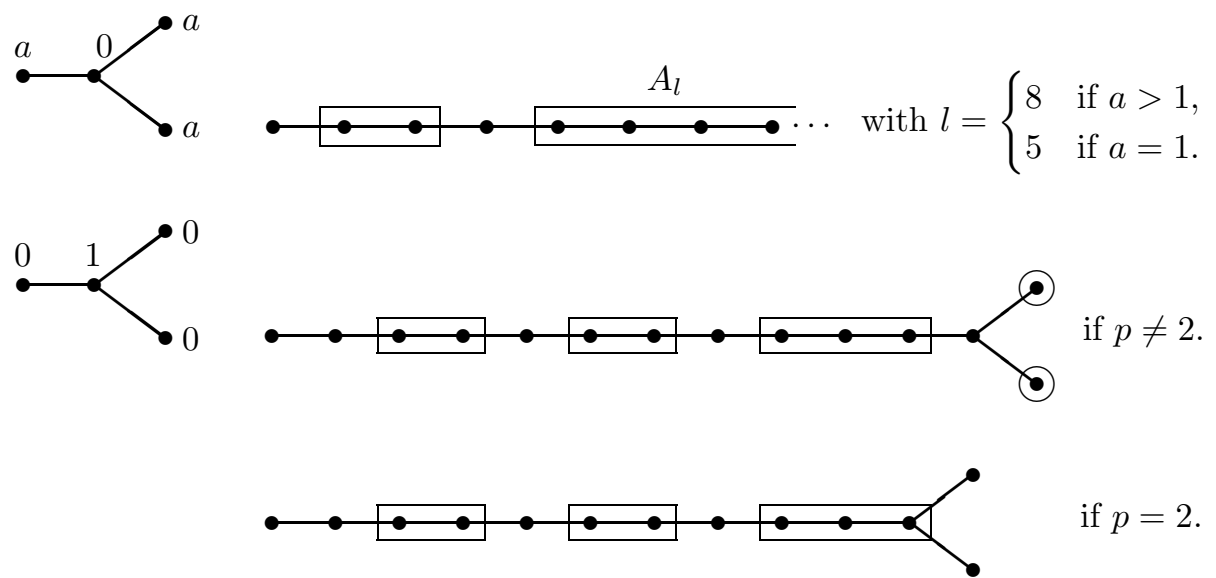

if $p=2$.

1) Assume $\delta=a \delta_{1}+a \delta_{3}+a \delta_{4}$. By the last Lemma, $\operatorname{dim}\left(V /\left[V, Q_{Y}\right]\right)=3$, so either $a_{2}=1$ or $a_{3}=1$, with all other $a_{i}=0$ for $\alpha_{i} \in \Pi\left(L_{Y}^{\prime}\right)$. By Lemma 2.4, $\operatorname{dim}\left(V^{2}\left(Q_{Y}\right)\right) \leq \operatorname{dim}\left(V^{2}\left(U_{X}\right)\right)=3 \operatorname{dim}\left(V_{1}^{2}\left(U_{X}\right)\right) \leq 3\left(4 \operatorname{dim}\left(V_{1}^{1}\left(U_{X}\right)\right)\right)=12$. If $a_{3}=1$, then in $V^{2}\left(Q_{Y}\right)$ we have either $\lambda-\alpha_{4}$ (if $a_{4} \neq 0$ ) or $\lambda-\alpha_{3}-\alpha_{4}$ (if $a_{4}=0$ ) as a high weight in $V^{2}\left(Q_{Y}\right)$. The $L_{Y}^{\prime}$-modules with these high weights have dimension greater than 12 , however, so in fact $a_{3}=0$.

If $a_{2}=1$, then similar calculations give contradictions to $\operatorname{dim}\left(V^{2}\left(Q_{Y}\right)\right) \leq 12$ if $0 \neq a_{1} \neq p-2$ or $a_{i} \neq 0$ for some $i>2$ or if $a>1$ and $a_{1} \neq 0$. So the only possibilities here are $a_{2}=1$ with $a_{i}=0$ for $i \neq 2$ (i.e. $\lambda=\lambda_{2}$ ) and $a=1, a_{1}=p-2$, $a_{2}=1$ with $a_{i}=0$ for $i>2$ (so $\left.\lambda=(p-2) \lambda_{1}+\lambda_{2}\right)$.

Assume $p=2$. Then, since $\delta$ is restricted by Lemma 2.6, $\delta=\delta_{1}+\delta_{3}+\delta_{4}$. In characteristic 2 , the $D_{4}$-module with this high weight has dimension 294 , so $Y$ has type $C_{147}$ or $D_{147}$. By the previous paragraph, we have $a_{1}=0$. But then $\lambda=\lambda_{2}$ has $\left(2^{147} \cdot 147 !\right) /\left(2 \cdot 2^{145} \cdot 145 !\right)=2 \cdot 147 \cdot 146=42,924$ conjugates. So $\operatorname{dim}(V) \geq 42,924$. The $T_{X}$-high weights of $V$ are restricted as well and not symmetric with respect to $s$, so the possibilities for these are very limited. In fact, any $D_{4}$-module with such a high weight has dimension at most 840 , but then $42,924 \leq \operatorname{dim}(V)=3 \operatorname{dim}\left(V_{1}\right) \leq 3 \cdot 840$ gives a contradiction. So we may assume $p \neq 2$.

Assume $\lambda=\lambda_{2}$; this implies $V \cong \bigwedge^{2} W$ ([7, II.2.15]). If $w \in W_{\delta}, w_{1} \in W_{\delta-\beta_{1}}$, $w_{2} \in W_{\delta-\beta_{3}}$, and $w_{3} \in W_{\delta-\beta_{4}}$, then $w \wedge w_{1}, w \wedge w_{2}$, and $w \wedge w_{3}$ are $T_{X}$-high weight vectors in $\bigwedge^{2} W$, of weights $(2 a-2) \delta_{1}+\delta_{2}+2 a \delta_{3}+2 a \delta_{4}, 2 a \delta_{1}+\delta_{2}+(2 a-2) \delta_{3}+2 a \delta_{4}$, and $2 a \delta_{1}+\delta_{2}+2 a \delta_{3}+(2 a-2) \delta_{4}$. Since we are assuming there are only three such $T_{X}$-high weights, $V$ must be the sum (as $X$-modules) of the three $D_{4}$-modules with these high weights.

Now switch parabolics, and embed the parabolic subgroup $P_{X}$ of $X$ corresponding to $\left\{\beta_{1}, \beta_{3}, \beta_{4}\right\}$ in a parabolic subgroup $P_{Y}$ of $Y$ via the $Q_{X}$-level construction. Then the factor $L_{1}$ of $L_{Y}^{\prime}$ corresponding to level 0 is the only simple factor to act nontrivially on $V /\left[V, Q_{Y}\right]$ and is of type $A_{l}$ with $l=(a+1)^{3}-1$. Now $V /\left[V, Q_{Y}\right] \cong_{L_{Y}^{\prime}}$ $\bigwedge^{2}\left(W /\left[W, Q_{Y}\right]\right)=\bigwedge^{2}$ (the natural module for $\left.L_{1}\right)$, and $\left.\left(V /\left[V, Q_{Y}\right]\right)\right|_{X}$ has the three 
high weights $(2 a-2,2 a, 2 a),(2 a, 2 a-2,2 a)$, and $(2 a, 2 a, 2 a-2)$. But this gives

$$
\left(\begin{array}{c}
(a+1)^{3} \\
2
\end{array}\right)=\operatorname{dim}\left(\bigwedge^{2}\left(W /\left[W, Q_{Y}\right]\right)\right)=3 \operatorname{dim}\left(V_{1} /\left[V_{1}, Q_{X}\right]\right)=3\left((2 a-1)(2 a+1)^{2}\right),
$$

and this equation in $a$ has no solutions in the positive integers. So $\lambda \neq \lambda_{2}$.

Finally, assume $a=1$ and $\lambda=(p-2) \lambda_{1}+\lambda_{2}$. Then $\left.\lambda_{1}\right|_{T_{X}}=\delta_{1}+\delta_{3}+\delta_{4}$ and as above we may assume that $\left.\lambda_{2}\right|_{T_{X}}=\left.\left(2 \lambda_{1}-\alpha_{1}\right)\right|_{T_{X}}=2\left(\delta_{1}+\delta_{3}+\delta_{4}\right)-\beta_{i}$ for some $i=1,3$, or 4 . So $\left.\lambda_{2}\right|_{T_{X}}$ is one of $\delta_{2}+2 \delta_{3}+2 \delta_{4}, 2 \delta_{1}+\delta_{2}+2 \delta_{4}$, or $2 \delta_{1}+\delta_{2}+2 \delta_{3}$. In any case, $\left.\lambda\right|_{T_{X}}=\left((p-2) \lambda_{1}+\lambda_{2}\right)_{T_{X}}=\left.(p-2) \lambda_{1}\right|_{T_{X}}+\left.\lambda_{2}\right|_{T_{X}}$ is a $T_{X}$-high weight of $V$ which is not restricted, contrary to our assumption. This eliminates the final possibility for case 1 .

2) If $\delta=\delta_{2}$, then $P_{Y}$ corresponds to the indicated nodes of the Dynkin diagram for $Y$, and the possibilities are that there is a label of 1 on one of the nodes in an $A_{2}$ factor of $L_{Y}^{\prime}$ or a label of 2 on one of the nodes corresponding to an $A_{1}$ factor. We have $\operatorname{dim}\left(V^{2}\left(Q_{Y}\right)\right) \leq 12$ by Lemma 2.4, and the same sorts of arguments as above give contradictions to this bound for any of these possibilities except $a_{4}=1$ and $a_{3}=1$; even further, we get contradictions to the bound unless a) $\lambda=a_{1} \lambda_{1}+a_{2} \lambda_{2}+\lambda_{3}$ or b) $\lambda=\lambda_{4}$.

a) Suppose $\delta=\delta_{2}$ and $\lambda=a_{1} \lambda_{1}+a_{2} \lambda_{2}+\lambda_{3}$. Then $\left.\lambda_{1}\right|_{T_{X}}=\delta_{2},\left.\lambda_{2}\right|_{T_{X}}=$ $\left(2 \lambda_{1}-\alpha_{1}\right)_{T_{X}}=2 \delta_{2}-\beta_{2}=\delta_{1}+\delta_{3}+\delta_{4}$, and $\left.\lambda_{3}\right|_{T_{X}}=\left(3 \lambda_{1}-2 \alpha_{1}-\alpha_{3}\right)_{T_{X}} \in\left\{2 \delta_{1}+\right.$ $\left.2 \delta_{3}, 2 \delta_{3}+2 \delta_{4}, 2 \delta_{1}+2 \delta_{4}\right\}$. So $\lambda$ restricts to $T_{X}$ as $a_{2} \delta_{1}+a_{1} \delta_{2}+\left(a_{2}+2\right) \delta_{3}+\left(a_{2}+2\right) \delta_{4}$ or one of its $s$-conjugates.

If we now embed the parabolic subgroup $P_{X}$ of $X$ corresponding to $\left\{\beta_{1}, \beta_{3}, \beta_{4}\right\} \subseteq$ $\Pi(X)$ in a parabolic subgroup $P_{Y}=Q_{Y} L_{Y}$ of $Y$, then the first nontrivial factor $L_{1}$ of $L_{Y}^{\prime}$ corresponds to $\left\{\alpha_{2}, \alpha_{3}, \ldots, \alpha_{8}\right\} \subseteq \Pi(Y)$ (since $Q_{X}$-level 1 has dimension 8). Here $L_{X}^{\prime}$ is a product of three groups of type $A_{1}$, and $\left(V /\left[V, Q_{Y}\right]\right)_{L_{X}^{\prime}}$ is the sum of three simple $L_{X}^{\prime}$-modules, with high weights $\left(a_{2}, a_{2}+2, a_{2}+2\right),\left(a_{2}+2, a_{2}, a_{2}+2\right)$, and $\left(a_{2}+2, a_{2}+2, a_{2}\right)$. So $\operatorname{dim}\left(V^{1}\left(Q_{Y}\right)\right)=3\left(a_{2}+1\right)\left(a_{2}+3\right)^{2}$. On the other hand, $V^{1}\left(Q_{Y}\right)$ is isomorphic as an $L_{1}$-module to the $A_{7}$-module $M$ with high weight $\gamma=a_{2} \gamma_{1}+\gamma_{2}$, where the $\gamma_{i}$ are the fundamental dominant weights for $A_{7}$. Assume that $a_{2}>15$. Then $\gamma$ has $\left(a_{2}-6\right) \gamma_{1}$ as a subdominant weight, and the $A_{7^{-}}$ module with high weight $\left(a_{2}-6\right) \gamma_{1}$ has $\left(\begin{array}{l}a_{2}+1 \\ a_{2}-6\end{array}\right)$ nonzero weights by Lemma 2.5. So $\operatorname{dim}\left(V^{1}\left(Q_{Y}\right)\right) \geq\left(\begin{array}{l}a_{2}+1 \\ a_{2}-6\end{array}\right)$, and $\left(\begin{array}{l}a_{2}+1 \\ a_{2}-6\end{array}\right)>3\left(a_{2}+1\right)\left(a_{2}+3\right)^{2}$ for $a_{2}>15$, which is a contradiction.

If $a_{2} \leq 15$, then we can check each case individually and we find more than $3\left(a_{2}+1\right)\left(a_{2}+3\right)^{2}$ weights in $M$, arriving at the same contradiction. So (a) is ruled out.

b) Suppose $\delta=\delta_{2}$ and $\lambda=\lambda_{4}$. We have $\left.\lambda_{4}\right|_{T_{X}}=\left(4 \lambda_{1}-3 \alpha_{1}-2 \alpha_{2}-\alpha_{3}\right)_{T_{X}} \in$ $\left\{4 \delta_{2}-3 \beta_{2}-\beta_{j}-\beta_{k} \mid j, k=1,3\right.$ or $\left.4, j \neq k\right\}=\left\{3 \delta_{1}+\delta_{3}+\delta_{4}, \delta_{1}+3 \delta_{3}+\delta_{4}, \delta_{1}+\delta_{3}+3 \delta_{4}\right\}$ as above. When we embed the parabolic subgroup $P_{X}$ of $X$ as above, we now have $\operatorname{dim}\left(\left.V^{1}\left(Q_{Y}\right)\right|_{L_{X}^{\prime}}\right)=3(2 \cdot 2 \cdot 4)=48$ on the one hand and $\operatorname{dim}\left(V^{1}\left(Q_{Y}\right)\right)=\left(\begin{array}{l}8 \\ 3\end{array}\right)=56$ on the other (since $V^{1}\left(Q_{Y}\right)$ is isomorphic to the $A_{7}$-module with high weight $\gamma_{3}$ ). Again we have a contradiction, and (b) is eliminated.

So there are no examples with $G=D_{4}\langle s\rangle$.

6.2. $G=X\langle s, t\rangle$. Assume $X=D_{4}$ with $G=X\langle s, t\rangle$. Lemma 6.2 tells us that we must have a $U_{X}$-level in $W$ of dimension 6 or less. Lemma 6.3 shows that there are few $T_{X}$-high weights $\delta$ of $W$ which allow such a level (other than level 1). We must consider level 1 for all $\delta$, level 2 for $\delta=b \delta_{2}$ or $\delta=\delta_{1}+\delta_{2}+\delta_{3}+\delta_{4}$, and the 
three possible $\delta$ not covered by the argument in the proof of that Lemma $\left(\delta_{2}, 2 \delta_{2}\right.$, and $\left.\delta_{1}+\delta_{3}+\delta_{4}\right)$.

As above, by Lemma 2.4 we have here $\operatorname{dim}\left(V^{2}\left(Q_{Y}\right)\right) \leq 4 \operatorname{dim}\left(V^{1}\left(Q_{Y}\right)\right)=24$. This rules out most of the remaining possibilities.

Assume $\delta=\delta_{1}+\delta_{3}+\delta_{4}$. Then we check the dimensions of the levels directly. With $W_{i}$ denoting $U_{X}$-level $i$ of $W$, we have $\operatorname{dim}\left(W_{0}\right)=1, \operatorname{dim}\left(W_{1}\right)=3, \operatorname{dim}\left(W_{2}\right)=6$, and $\operatorname{dim}\left(W_{i}\right) \geq 7$ for $i \geq 3$. So $P_{Y}$ corresponds to the indicated nodes in the Dynkin diagram for $Y$ in this picture:

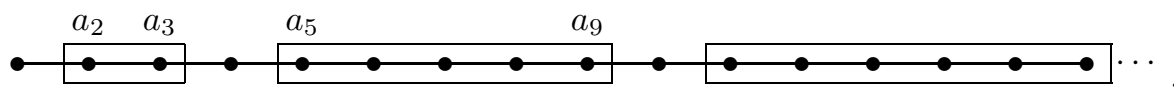

Lemma 6.2 tells us that $\operatorname{dim}\left(V^{1}\left(Q_{Y}\right)\right)=6$; the ways in which this can happen given the dimensions of $U_{X}$-levels above are a) $a_{2}=2$ and $a_{i}=0$ for $i \neq 2$, $\alpha_{i} \in \Pi(Y)-\Pi\left(L_{Y}^{\prime}\right)$; b) $a_{3}=2$ and $a_{i}=0$ for $\left.i \neq 3, \alpha_{i} \in \Pi(Y)-\Pi\left(L_{Y}^{\prime}\right) ; \mathrm{c}\right) a_{5}=1$ and $a_{i}=0$ for $i \neq 5, \alpha_{i} \in \Pi(Y)-\Pi\left(L_{Y}^{\prime}\right)$; and d) $a_{9}=1$ and $a_{i}=0$ for $i \neq 9$, $\alpha_{i} \in \Pi(Y)-\Pi\left(L_{Y}^{\prime}\right)$. We consider each possibility in turn (the arguments below must be modified slightly in some small characteristics).

a) If $a_{2}=2$, then either $\lambda-\alpha_{1}$ (if $a_{1} \neq 0$ ) or $\lambda-\alpha_{2}-\alpha_{1}$ (if $a_{1}=0$ ) is a high weight in $V^{2}\left(Q_{Y}\right)$, giving a composition factor there of dimension at least 7 , and either $\lambda-\alpha_{4}$ (if $a_{4} \neq 0$ ) or $\lambda-\alpha_{2}-\alpha_{3}-\alpha_{4}$ (if $a_{4}=0$ ) is another high weight, giving a factor of dimension at least 18 . This contradicts $\operatorname{dim}\left(V^{2}\left(Q_{Y}\right)\right) \leq 24$. So this case does not arise.

b) If $a_{3}=2$, then $\lambda-\alpha_{4}$ (if $a_{4} \neq 0$ ) or $\lambda-\alpha_{3}-\alpha_{4}$ gives a composition factor in $V^{2}\left(Q_{Y}\right)$ of dimension at least 42 ; this again is a contradiction.

c) If $a_{5}=1$, then either $\lambda-\alpha_{4}$ (if $a_{4} \neq 0$ ) or $\lambda-\alpha_{4}-\alpha_{5}$ (if $a_{4}=0$ ) is a high weight in $V^{2}\left(Q_{Y}\right)$, giving $\operatorname{dim}\left(V^{2}\left(Q_{Y}\right)\right) \geq 45$. This again contradicts $\operatorname{dim}\left(V^{2}\left(Q_{Y}\right)\right) \leq 24$.

d) Similarly, here we obtain a contradiction since either $\lambda-\alpha_{10}$ or $\lambda-\alpha_{9}-\alpha_{10}$ is a high weight in $V^{2}\left(Q_{Y}\right)$, giving $\operatorname{dim}\left(V^{2}\left(Q_{Y}\right)\right) \geq 105$.

So the embedding $\delta=\delta_{1}+\delta_{3}+\delta_{4}$ gives no examples.

Almost identical arguments rule out all possibilities for $\delta=2 \delta_{2}$, so this embedding gives no examples.

For $\delta=\delta_{2}$, there are many more possibilities for the labelling of $\lambda$ on $\Pi\left(L_{Y}^{\prime}\right)$. Here the picture of $P_{Y}$ is

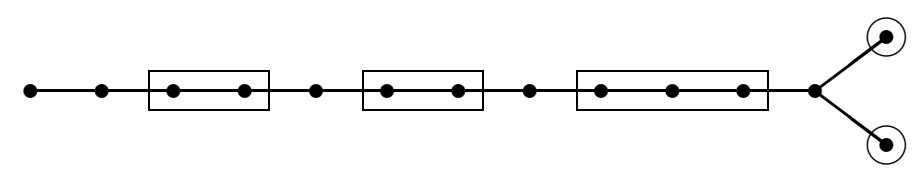

for $p \neq 2$, and

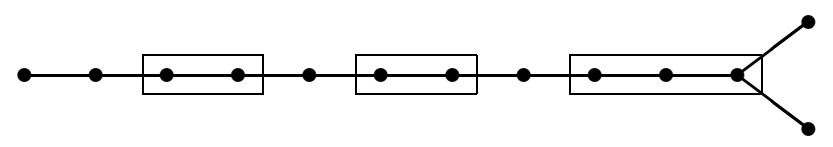

for $p=2$ (in [9] it is calculated that this embedding is actually into $D_{13}$, not $\left.C_{13}\right)$. Once again, however, arguments like those above give contradictions to $\operatorname{dim}\left(V^{2}\left(Q_{Y}\right)\right) \leq 24$ for all possible $\lambda$ except perhaps $\lambda=a_{1} \lambda_{1}+a_{2} \lambda_{2}+2 \lambda_{3}$, with 
$a_{1} a_{2}=0$. So we may assume $\lambda$ has this form and $p \neq 2$ since $\lambda$ has $\lambda_{3}$-coefficient 2 .

If $a_{1}=0 \neq a_{2}$ and $\lambda=a_{2} \lambda_{2}+2 \lambda_{3}$, then we use the $s$-stable minimal parabolic subgroup $P_{X}$ of $X$ corresponding to $\left\{\beta_{2}\right\} \subseteq \Pi(X)$. We embed $P_{X}$ in a parabolic subgroup $P_{Y}$ of $Y$ corresponding to the indicated nodes in the picture

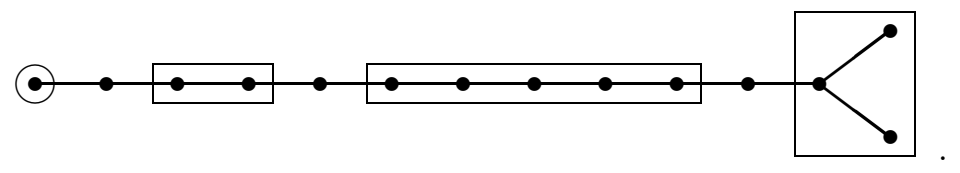

Now by Lemma 2.4, we have $\operatorname{dim}\left(V^{2}\left(Q_{Y}\right)\right) \leq 6 \operatorname{dim}\left(V^{1}\left(Q_{Y}\right)\right)=36$. But $\lambda-\alpha_{2}$ and $\lambda-\alpha_{3}-\alpha_{4}-\alpha_{5}$ are high weights in $V^{2}\left(Q_{Y}\right)$, giving composition factors of dimension 20 (if $p \neq 3$ ) and 18, respectively; this is a contradiction (if $p=3$, there is another composition factor of high weight $\lambda-\alpha_{2}-\alpha_{3}$ which we must include to obtain the contradiction).

TABle 1. Examples arising from the connected case.

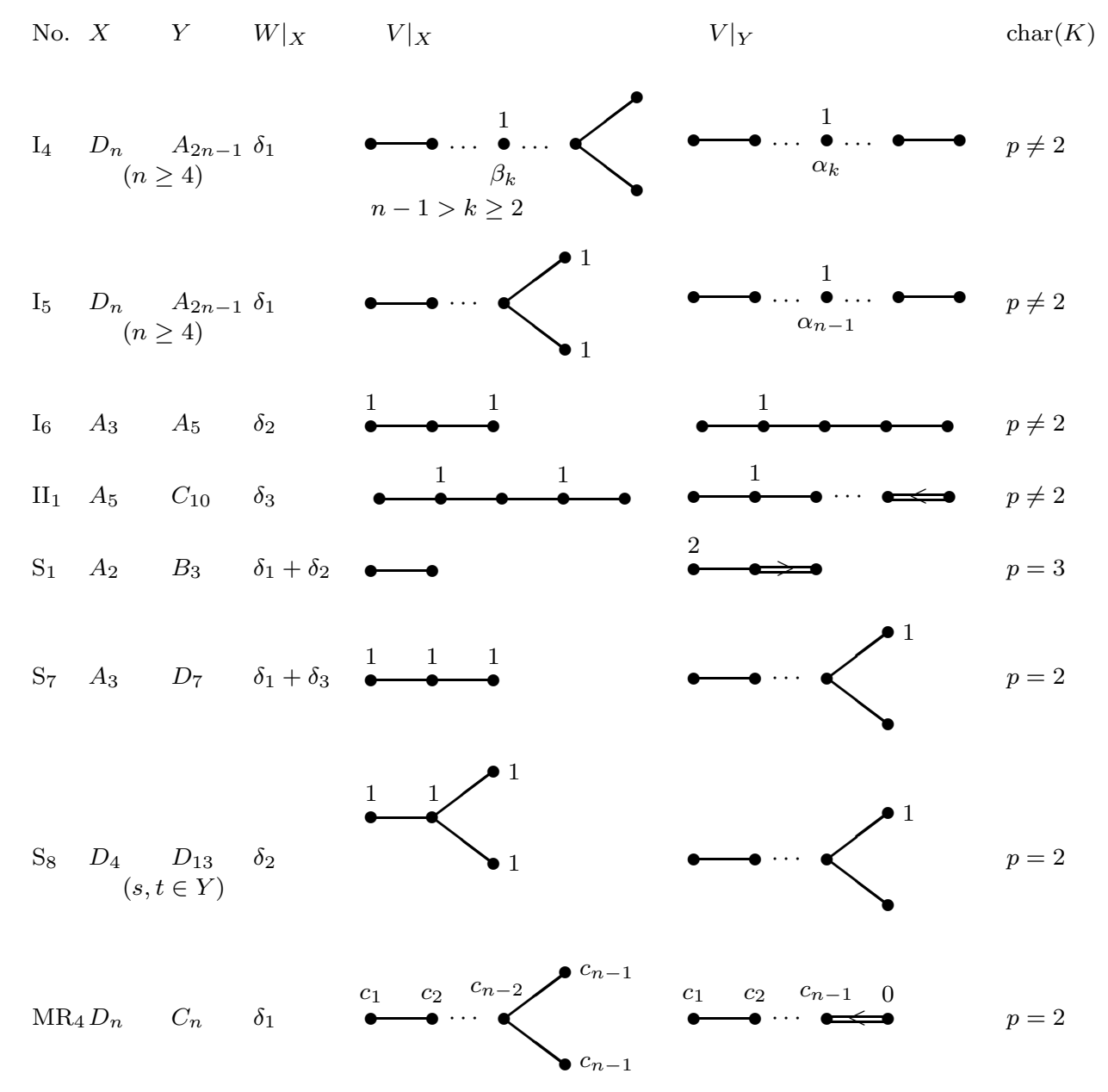


TABLE 2. New Examples.

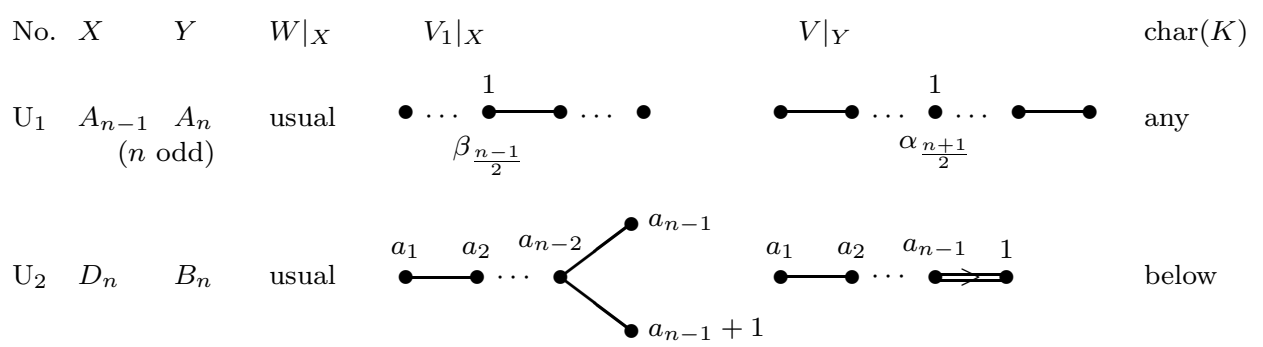

with $a_{i}+a_{j} \equiv i-j(\bmod p)$ whenever $a_{i}$ and $a_{j}$ are non-zero coefficients with only 0 's between them and $i<j<n ; 2 a_{i} \equiv-2(n-i)-1(\bmod p)$ for $a_{i}$ the last non-zero coefficient before $a_{n}=1$.
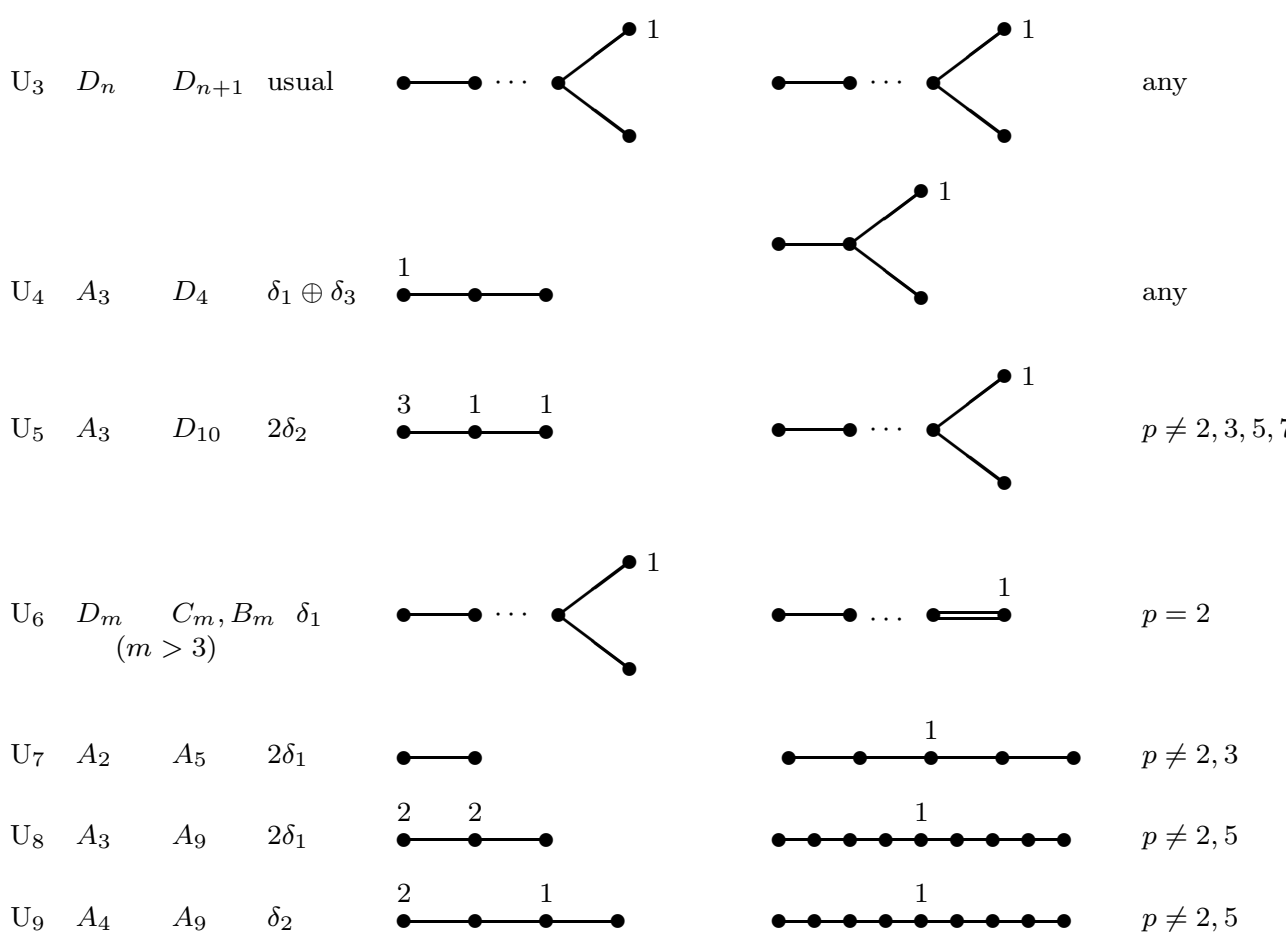

If $a_{2}=0$ and $a_{1} \neq 0$ (so $\lambda=a_{1} \lambda_{1}+2 \lambda_{3}$ ), then we can examine the embedding of the $s$-stable parabolic subgroup of $X$ corresponding to $\left\{\beta_{1}, \beta_{3}, \beta_{4}\right\} \subseteq \Pi\left(L_{Y}^{\prime}\right)$ in a parabolic subgroup $P_{Y}=Q_{Y} L_{Y}$ of $Y$. Now the first nontrivial factor $L_{1}$ of $L_{Y}^{\prime}$ corresponds to $\left\{\alpha_{2}, \ldots, \alpha_{8}\right\}$ (as $Q_{X}$-level 1 has dimension 8), and the second factor $L_{2}$ corresponds to $\left\{\alpha_{10}, \ldots, \alpha_{14}\right\}$. The first, $L_{1}$, is the only one to act nontrivially on $V^{1}\left(Q_{Y}\right)$, which is isomorphic to the $A_{7}$-module with high weight $2 \gamma_{2}$ (where $\gamma_{i}$ are the fundamental dominant weights of $A_{7}$ ). We find (using the Weyl character formula and the Andersen-Jantzen sum formula) that $\operatorname{dim}\left(V^{1}\left(Q_{Y}\right)\right)=336$ if $p \neq 3$ 
and $\operatorname{dim}\left(V^{1}\left(Q_{Y}\right)\right)=266$ if $p=3$. By Lemma 2.4, we have $\operatorname{dim}\left(V^{2}\left(Q_{Y}\right)\right) \leq$ $8 \operatorname{dim}\left(V^{1}\left(Q_{Y}\right)\right)$. With $\lambda$ as above, the two high weights $\lambda-\alpha_{1}$ and $\lambda-\alpha_{3}-\cdots-\alpha_{9}$ in $V^{2}\left(Q_{Y}\right)$ give, for $p \neq 3$, two composition factors, each of dimension 1680; for $p=3$, these two composition factors have dimension 1624 and 1120, respectively. This contradicts $\operatorname{dim}\left(V^{2}\left(Q_{Y}\right)\right) \leq 8 \operatorname{dim}\left(V^{1}\left(Q_{Y}\right)\right)$.

The remaining possibility is $\lambda=2 \lambda_{3}$. We know that $\left.\lambda_{1}\right|_{T_{X}}=\delta_{2},\left.\alpha_{1}\right|_{T_{X}}=\beta_{2}$ by $[9,3.4(\mathrm{i})]$, and $\left.\alpha_{2}\right|_{T_{X}}=\beta_{i}$ for $i=1,3$ or 4 , by the same arguments used in the proof of $[9,3.4]$. Since $\lambda_{3}=3 \lambda_{1}-2 \alpha_{1}-\alpha_{2}$, this gives $\left.\lambda_{3}\right|_{T_{X}}$ of the form $2 \delta_{i}+2 \delta_{j}$ for $(i, j)=(1,3),(1,4)$, or $(3,4)$. But we have noted that the $\delta_{1^{-}}, \delta_{3^{-}}$, and $\delta_{4}$-coefficients of $\left.\lambda\right|_{T_{X}}$ must be distinct in this case $G=D_{4}\langle s, t\rangle$, since $V$ is irreducible as a $G$-module. So the case $\delta=\delta_{2}$ does not occur.

Remaining to be considered are level 1 for all $\delta$, and level 2 for $\delta=\delta_{1}+\delta_{2}+\delta_{3}+\delta_{4}$ or $\delta=b \delta_{2}$ (with $b>1$, as the case $\delta=\delta_{2}$ was examined thoroughly above).

First we consider level 2. If $\delta=\delta_{1}+\delta_{2}+\delta_{3}+\delta_{4}$, then $W_{2}$ in fact has dimension 9 unless $p=3$, in which case it has dimension 6 and we have the possibilities $a_{6} \neq 0$ and $a_{10} \neq 0$. But these cases are easily ruled out by arguments based on $\operatorname{dim}\left(V^{2}\left(Q_{Y}\right)\right) \leq 24$, as in several cases above. If $\delta=b \delta_{2}$, then $\operatorname{dim}\left(W_{2}\right)=4$ and we have the possibility $a_{4} \neq 0$; this is again ruled out by the same sorts of arguments.

Finally, assume $\lambda$ has a nonzero coefficient for a fundamental weight corresponding to some $\alpha_{i}$ in $\Pi\left(L_{1}\right)$, where $L_{1}$ is the simple factor of $L_{Y}^{\prime}$ corresponding to the $U_{X}$-level 1 of $W$ (in other words, we are in the remaining level 1 case). Then if $a \neq 0 \neq b, \operatorname{dim}\left(W_{1}\right)=4$ and we have the possibility $a_{3}=1$. If $a \neq 0=b$, then $\operatorname{dim}\left(W_{1}\right)=3$ and we have the possibilities $a_{2}=2$ and $a_{3}=2$. These all fall quickly to arguments based on the fact that $\operatorname{dim}\left(V^{2}\left(Q_{Y}\right)\right) \leq 24$ as above. If $b \neq 0=a$, then $\operatorname{dim}\left(W_{1}\right)=1$ and $L_{1}$ is trivial, so we do not have a possibility. This rules out level 1.

We have eliminated all possible high weights $\delta$ for $W$. This completes the proof of Theorem 6.1, which in turn completes the proof of Theorem 1.

\section{REFERENCES}

1. Henning Haahr Andersen, Filtrations of cohomology modules for Chevalley groups, Ann. Sci. École Norm. Sup. (4) 16 (1983), 495-528. MR 85k:20128

2. Eugene B. Dynkin, Maximal subgroups of the classical groups, Amer. Math. Soc. Transl. Ser. 26 (1957), 245-378. MR 14:244d

3. 6 (1957), 111-244. MR 13:904c

4. Ben Ford, Overgroups of irreducible linear groups, I, J. Algebra 181 (1996), 26-69. MR 97b:20067

5. James E. Humphreys, Introduction to Lie algebras and representation theory, Graduate Texts in Mathematics, vol. 9, Springer, New York/Heidelberg/Berlin, 1972. MR 48:2197

6. Jens Carsten Jantzen, Weyl modules for groups of Lie type, Finite Simple Groups II (New York), Academic Press, 1980, pp. 291-300.

7. — Representations of algebraic groups, Academic Press, Boston, 1987. MR 89c:20001

8. Alexander A. Premet, Weights of infinitesimally irreducible representations of Chevalley groups over a field of prime characteristic, Math. USSR-Sb. 61 (1988), 167-183. MR 88h:20051

9. Gary M. Seitz, The maximal subgroups of classical algebraic groups, Mem. Amer. Math. Soc. 67 (1987), no. 365, 1-286. MR 88g:20092

10. - Maximal subgroups of exceptional algebraic groups, Mem. Amer. Math. Soc. 90 (1991), no. 441, 1-197. MR 91g:20038

11. Robert Steinberg, Lectures on Chevalley groups, Yale University, 1968. MR 57:6215 
12. Irene D. Suprunenko, The invariance of the weight system of irreducible representations of algebraic groups and Lie algebras of type $A_{l}$ with restricted highest weights, under reduction modulo p, Vestsī Akad. Navuk BSSR Ser. Fīz.-Mat. Navuk 2 (1983), 18-22 (Russian).

13. Donna M. Testerman, Irreducible subgroups of exceptional algebraic groups, Mem. Amer. Math. Soc. 75 (1988), no. 390, 1-190. MR 90a:20082

Department of Mathematics, University of Washington, Box 354350, Seattle, WashINGTON 98195-4350

Current address: Department of Mathematics, Sonoma State University, Rohnert Park, California 94928

E-mail address: ben.ford@sonoma.edu 